\title{
A Chiral Acrylate Equivalent for Metal-Free Diels-Alder Reactions: Endo-2-Acryloylisoborneol.
}

\author{
Claudio Palomo, ${ }^{\# *}$ Mikel Oiarbide, ${ }^{\#}$ Jesús M. García, ${ }^{\dagger}$ Alberto González, ${ }^{\dagger}$ Ainara \\ Lecumberri, ${ }^{\dagger}$ Anthony Linden. ${ }^{\S}$ \\ ${ }^{\#}$ Departamento de Química Orgánica I, Facultad de Química, Universidad del País Vasco, Apdo. 1072, \\ 20080 San Sebastián, Spain; 'Departamento de Química Aplicada, Universidad Pública de Navarra, \\ Campus de Arrosadía 31006 Pamplona, Spain; and ${ }^{\S}$ Organisch-chemisches Institut der Universität Zürich, \\ Winterthurerstrasse 190, CH-8057, Zürich, Switzerland.
}

\section{Supporting Information}

\section{General.}

All reactions were carried out under an atmosphere of nitrogen in oven or flame-dried glassware with magnetic stirring. Solvents were distilled prior to use. Tetrahydrofuran (THF) and diethyl ether were distilled from sodium metal/benzophenone ketyl. Dichloromethane $\left(\mathrm{CH}_{2} \mathrm{Cl}_{2}\right)$ was distilled from calcium hydride. Cyclopentadiene was distilled by cracking dicyclopentadiene prior to use. Other dienes were used without purification. Purification of reaction products was carried out by flash chromatography using silicagel 60 (230-400 mesh). Analytical thin layer chromatography was performed on $0.25 \mathrm{~mm}$ silica gel $60-\mathrm{F}$ plates. Visualization was accomplished with UV light and p-anisaldehyde solution followed by heating. Melting points were measured with a Mettler FP 80 melting point apparatus and are uncorrected. Infrared spectra were recorded on a Nicolet Avatar 360 FT-IR spectrometer. ${ }^{1} \mathrm{H}$ NMR and ${ }^{13} \mathrm{C}$ NMR spectra were recorded on a Varian Gemini-200 spectrometer and are reported in ppm from $\mathrm{CHCl}_{3}$. Combustion analyses were performed on a Carlo Erba CHNS-O EA1108 elemental analyzer. Optical rotations were measured at $25 \pm 0.2{ }^{\circ} \mathrm{C}$ in methylene chloride unless otherwise stated. Analytical high performance liquid chromatography (HPLC) was performed on a Waters chromatograph equipped with a diode array UV detector, using the columns specified to the end of this report.

(1R)-2-endo-propenoyl-1,7,7-trimethylbicyclo[2.2.1] heptan-2-ol. $\underline{3}$

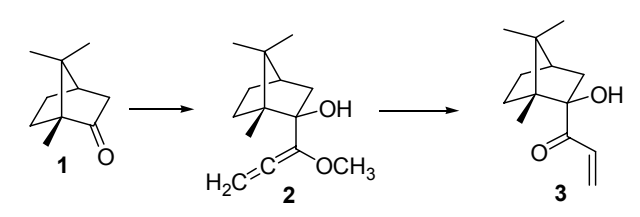

Methoxyallene $^{1}(2.94 \mathrm{~g}, 42 \mathrm{mmol})$ was added to a solution of $\mathrm{nBuLi}(2.5 \mathrm{M}$ in hexanes, $16 \mathrm{~mL}, 40 \mathrm{mmol})$ cooled to $-30{ }^{\circ} \mathrm{C}$ under a nitrogen atmosphere. After stirring for 30 minutes at the same temperature, TMDA $(6.1 \mathrm{~mL}, 40 \mathrm{mmol})$ and a solution of (1R)-(+)-camphor

\footnotetext{
${ }^{1}$ (a) Brandsma, L.; Verkruijsse, H. D. Synthesis of Acetylenes, Allenes and Cumulenes; Elsevier: New York, 1981. (b) Tius, M. A. In Encyclopedia of Reagent for Organic Synthesis; Paquette, L. A., Ed.; Wiley: New York, 1995, Vol. 5, pp.3316-3320. (c) Zimmer, R.; Khan, F. A. J. prak.Chem.1996, 338, 92-94. (d) Pulz, R. Synlett 2000, 1697.
} 
(3.04 g, $20 \mathrm{mmol})$ in THF $(2.6 \mathrm{~mL})$ were added dropwise. The resulting mixture was allowed to stir for 2 hours at $-30{ }^{\circ} \mathrm{C}$ and then was quenched with a saturated aqueous solution of $\mathrm{NH}_{4} \mathrm{Cl}(20 \mathrm{~mL})$. The aqueous layer was extracted with $\mathrm{CH}_{2} \mathrm{Cl}_{2}(2 \times 20 \mathrm{~mL})$ and the combined organics were dried over $\mathrm{MgSO}_{4}$, and the solvent was removed under reduced pressure. The product 2 was purified by distillation (about $10 \%$ of starting camphor was separated) and collected as a colorless liquid. Yield of (1R)-2-endo-1-methoxy-1,2-propanodienyl-1,7,7-trimethylbicyclo[2.2.1] heptan-2-ol (2): $3.33 \mathrm{~g}$ (75\%); b.p. $70-72^{\circ} \mathrm{C} / 0.1$ torr; $[\alpha]_{\mathrm{D}}^{25}+38.0\left(c 1.0, \mathrm{Cl}_{2} \mathrm{CH}_{2}\right)$; IR (neat, $\left.\mathrm{cm}^{-1}\right) 3585,2950,2924,2868,1951,1066,876 ;{ }^{1} \mathrm{H}$ NMR $\left(\mathrm{CDCl}_{3}\right) \delta 5.55(\mathrm{~d}, 1 \mathrm{H}, \mathrm{HC}=\mathrm{C}, \mathrm{J}=7.7 \mathrm{~Hz})$, $5.47(\mathrm{~d}, 1 \mathrm{H}, \mathrm{HC}=\mathrm{C}, \mathrm{J}=7.7 \mathrm{~Hz}), 3.44\left(\mathrm{~s}, 3 \mathrm{H}, \mathrm{OCH}_{3}\right), 2.80(\mathrm{~s}, 1 \mathrm{H}, \mathrm{OH}), 2.06-1.95(\mathrm{~m}, 1 \mathrm{H}, \mathrm{HCH})$, 1.80-1.73 (d, 1H, HCH, J=13Hz) 1.69-1.57 (m, 1H, HCH), 1.48-1.29 (m, 2H, HCH), $1.12(\mathrm{~s}, 3 \mathrm{H}$, $\left.\mathrm{CH}_{3}\right), 1.08-0.88(\mathrm{~m}, 2 \mathrm{H}, \mathrm{HCH}), 1.00\left(\mathrm{~s}, 3 \mathrm{H}, \mathrm{CH}_{3}\right), 0.80\left(\mathrm{~s}, 3 \mathrm{H}, \mathrm{CH}_{3}\right) ;{ }^{13} \mathrm{C} \mathrm{NMR}\left(\mathrm{CDCl}_{3}\right) \delta 197.5$, $138.9,92.1,82.0,56.4,52.9,49.8,44.8,42.5,31.5,26.6,21.1,20.9,11.3$. The distillate product was dissolved in $\mathrm{CH}_{2} \mathrm{Cl}_{2}(30 \mathrm{~mL})$ and washed twice with $1 \mathrm{M} \mathrm{HCl}(15 \mathrm{~mL})$. The organic layer was dried over $\mathrm{MgSO}_{4}$, filtered and the solvent was removed under reduced pressure to give the vinyl ketone 3 as a white solid. Purification was effected by flash silica gel column chromatography using a 1:30 ethyl acetate/hexane mixture as the eluant. Yield of (1R)-2-endo-propenoyl-1,7,7trimethylbicyclo[2.2.1]heptan-2-ol (3): $3.12 \mathrm{~g}\left(100 \%\right.$ from 2); m.p. 72.5-73.0 ${ }^{\circ} \mathrm{C}$; $[\alpha]_{\mathrm{D}}^{25}-34.5(c 1.0$, $\left.\mathrm{Cl}_{2} \mathrm{CH}_{2}\right)$; IR (neat, $\left.\mathrm{cm}^{-1}\right) 3488(\mathrm{OH}), 1685(\mathrm{CO}) ;{ }^{1} \mathrm{H} \mathrm{NMR}\left(\mathrm{CDCl}_{3}\right) \delta 6.88(\mathrm{dd}, 1 \mathrm{H}, \mathrm{HC}=\mathrm{C}$, $\left.\mathrm{J}=10.6 \mathrm{~Hz}, \mathrm{~J}^{\prime}=17.2 \mathrm{~Hz}\right), 6.34\left(\mathrm{dd}, 1 \mathrm{H}, \mathrm{HCH}=\mathrm{C}, \mathrm{J}=2.2 \mathrm{~Hz}, \mathrm{~J}^{\prime}=16.9 \mathrm{~Hz}\right), 5.67(\mathrm{dd}, 1 \mathrm{H}, \mathrm{HCH}=\mathrm{C}$, $\left.\mathrm{J}=2.2 \mathrm{~Hz}, \mathrm{~J}^{\prime}=10.3 \mathrm{~Hz}\right), 2.88(\mathrm{~s}, 1 \mathrm{H}, \mathrm{OH}), 2.34-2.25(\mathrm{~m}, 1 \mathrm{H}, \mathrm{HCH}), 1.88-1.64(\mathrm{~m}, 2 \mathrm{H}, \mathrm{HCH}), 1.44-$

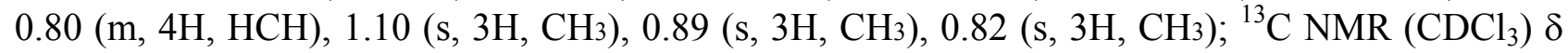
201.6, 131.8, 128.7, 86.9, 52.6, 50.6, 45.2, 41.3, 30.2, 26.5, 20.9, 20.4, 10.6; Anal. calcd for $\mathrm{C}_{13} \mathrm{H}_{20} \mathrm{O}_{2}(208.33) \mathrm{C}, 74.94 ; \mathrm{H}, 9.70$. Found: C, 74.85; H, 10.01 .

\section{(1R)-2-endo-propenoyl-2-exo-methoxy-1,7,7-trimethylbicyclo[2.2.1]heptane. $\underline{4}$}

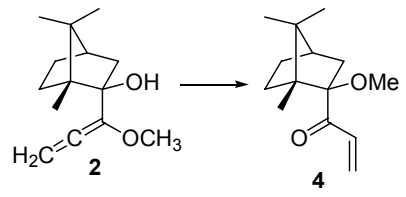

Methyl iodide $(0.51 \mathrm{~mL}, 8 \mathrm{mmol})$ was added to a solution of $\mathrm{NaH}$ $(0.20 \mathrm{~g}, 8 \mathrm{mmol})$ in dry DMF $(12 \mathrm{~mL})$ cooled to $-50{ }^{\circ} \mathrm{C}$ under a nitrogen atmosphere. Then a solution of allene 2 reported above $(0.889 \mathrm{~g}, 4 \mathrm{mmol})$ in $\operatorname{DMF}(4 \mathrm{~mL})$ was added at the same temperature and the mixture was stirred at $0{ }^{\circ} \mathrm{C}$ for 1 hour. The reaction was quenched with a saturated aqueous solution of $\mathrm{NH}_{4} \mathrm{Cl}(10 \mathrm{~mL})$ and then was extracted twice with $\mathrm{Et}_{2} \mathrm{O}(15 \mathrm{~mL})$, and the combined organic layers were washed with water $(2 \times 10 \mathrm{~mL})$. The organic layer was dried over $\mathrm{MgSO}_{4}$, and the solvent was removed under reduced pressure. The residue thus obtained was dissolved in $\mathrm{Et}_{2} \mathrm{O}(8 \mathrm{~mL})$ and a $5 \%$ aqueous solution of $\mathrm{H}_{2} \mathrm{SO}_{4}(8 \mathrm{~mL})$ was added at $0{ }^{\circ} \mathrm{C}$ and the mixture was stirred at the same temperature for 16 hours. The layers were separated, and the aqueous layer was extracted with $\mathrm{CH}_{2} \mathrm{Cl}_{2}(2 \times 10 \mathrm{~mL})$. The combined organics were dried over $\mathrm{MgSO}_{4}$, and the solvent was removed under reduced pressure. Purification of the product 4 was effected by flash silica gel column chromatography using a 1:30 ethyl acetate/hexane mixture as the eluant. Yield of (1R)-2-endo-propenoyl-2-exo-methoxy-1,7,7-trimethylbicyclo[2.2.1]heptane (4): $0.534 \mathrm{~g}$ (60\% from 2); oil; $[\alpha]_{\mathrm{D}}^{25}+27.0$ (c 1.0, $\mathrm{Cl}_{2} \mathrm{CH}_{2}$ ); IR (neat, $\mathrm{cm}^{-1}$ ) 1691 (CO); ${ }^{1} \mathrm{H} \mathrm{NMR}$ $\left(\mathrm{CDCl}_{3}\right) \delta 6.98\left(\mathrm{dd}, 1 \mathrm{H}, \mathrm{HC}=\mathrm{C}, \mathrm{J}=10.3 \mathrm{~Hz}, \mathrm{~J}^{\prime}=17.2 \mathrm{~Hz}\right), 6.33(\mathrm{dd}, 1 \mathrm{H}, \mathrm{HCH}=\mathrm{C}, \mathrm{J}=2.2 \mathrm{~Hz}$, $\left.\mathrm{J}^{\prime}=17.6 \mathrm{~Hz}\right), 5.64\left(\mathrm{dd}, 1 \mathrm{H}, \mathrm{HCH}=\mathrm{C}, \mathrm{J}=2.2 \mathrm{~Hz}, \mathrm{~J}^{\prime}=10.3 \mathrm{~Hz}\right), 3.00\left(\mathrm{~s}, 3 \mathrm{H}, \mathrm{CH}_{3}\right), 2.16(\mathrm{~d}, 1 \mathrm{H}, \mathrm{HCH}$, $\mathrm{J}=12.8), 1.92-1.55(\mathrm{~m}, 3 \mathrm{H}, \mathrm{HCH}), 1.35-1.10(\mathrm{~m}, 2 \mathrm{H}, \mathrm{HCH}), 1.00\left(\mathrm{~s}, 3 \mathrm{H}, \mathrm{CH}_{3}\right), 0.98\left(\mathrm{~s}, 3 \mathrm{H}, \mathrm{CH}_{3}\right)$, 0.87-0.75 (m, 1H, HCH), $0.81(\mathrm{~s}, 3 \mathrm{H}, \mathrm{CH}) ;{ }^{13} \mathrm{C} \mathrm{NMR}\left(\mathrm{CDCl}_{3}\right) \delta 201.6,132.5,127.9,93.5,52.1$, $51.1,50.6,45.2,33.3,30.6,26.0,20.6,20.6,11.3$. 
(1R)-2-endo-propenoyl-2-exo-trimethylsilyloxy-1,7,7-trimethylbicyclo[2.2.1]-heptane. $\underline{5}$

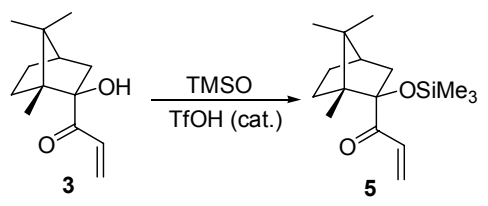
added and the resulting solution was washed with a saturated aqueous solution of $\mathrm{NaHCO}_{3}(2 \times 15$ $\mathrm{mL}$ ). The organic layer was dried over $\mathrm{MgSO}_{4}$, filtered and the solvent was removed under reduced pressure. Hexane $(15 \mathrm{~mL})$ was added to the oily residue and the resulting white solid was filtered and washed with additional hexane $(15 \mathrm{~mL})$. The solvent was evaporated and the crude product was purified by column chromatography (eluant: ethyl acetate/hexane 1:30). Yield (1R)-2-endopropenoyl-2-exo-trimethylsilyloxy-1,7,7-trimethylbicyclo[2.2.1]-heptane (5): $2.78 \mathrm{~g}$ (99\%); oil; $[\alpha]_{\mathrm{D}}^{25}-15.0\left(c\right.$ 1.0, $\left.\mathrm{Cl}_{2} \mathrm{CH}_{2}\right)$; IR (neat, $\left.\mathrm{cm}^{-1}\right) 1696(\mathrm{CO}) ;{ }^{1} \mathrm{H} \mathrm{NMR}\left(\mathrm{CDCl}_{3}\right) \delta 6.92(\mathrm{dd}, 1 \mathrm{H}, \mathrm{HC}=\mathrm{C}$, $\left.\mathrm{J}=10.3 \mathrm{~Hz}, \mathrm{~J}^{\prime}=17.2 \mathrm{~Hz}\right), 6.35\left(\mathrm{dd}, 1 \mathrm{H}, \mathrm{HCH}=\mathrm{C}, \mathrm{J}=2.2 \mathrm{~Hz}, \mathrm{~J}^{\prime}=17.2 \mathrm{~Hz}\right), 5.68(\mathrm{dd}, 1 \mathrm{H}, \mathrm{HCH}=\mathrm{C}$, $\left.\mathrm{J}=2.2 \mathrm{~Hz}, \mathrm{~J}^{\prime}=10.3 \mathrm{~Hz}\right), 2.57(\mathrm{~d}, 1 \mathrm{H}, \mathrm{HCH}, \mathrm{J}=12.5), 1.90-1.74(\mathrm{~m}, 1 \mathrm{H}, \mathrm{HCH}), 1.67-1.40(\mathrm{~m}, 1 \mathrm{H}$, $\mathrm{HCH}), 1.37-0.68(\mathrm{~m}, 4 \mathrm{H}, \mathrm{HCH}), 1.09\left(\mathrm{~s}, 3 \mathrm{H}, \mathrm{CH}_{3}\right), 1.00\left(\mathrm{~s}, 3 \mathrm{H}, \mathrm{CH}_{3}\right), 0.85\left(\mathrm{~s}, 3 \mathrm{H}, \mathrm{CH}_{3}\right), 0.08(\mathrm{~s}$, $\left.9 \mathrm{H}, 3 \mathrm{CH}_{3}\right) ;{ }^{13} \mathrm{C} \mathrm{NMR}\left(\mathrm{CDCl}_{3}\right) \delta 199.8,133.2,127.6,90.0,51.8,50.9,45.5,40.0,30.0,25.8,21.0$, $20.4,1.7$.

(1R)-2-endo-2'-butenoyl-1,7,7-trimethylbicyclo[2.2.1]heptan-2-ol. $\underline{6^{2}}$

To a solution of $2(0.222 \mathrm{~g}, 1 \mathrm{mmol})$ in THF $(4 \mathrm{~mL})$ at $-78^{\circ} \mathrm{C}, \mathrm{nBuLi}(2.5 \mathrm{M}$ in hexanes, $1.2 \mathrm{~mL}, 3$ mmol) was added and the reaction was stirred at $-25{ }^{\circ} \mathrm{C}$ for $4 \mathrm{~h}$. Then, MeI $(0.21 \mathrm{~mL}, 3.5 \mathrm{mmol})$ was added and the mixture was stirred for $2 \mathrm{~h}$ at the same temperature. The reaction was quenched with $1 \mathrm{~N} \mathrm{HCl}(5 \mathrm{~mL})$ and extracted with $\mathrm{CH}_{2} \mathrm{Cl}_{2}(2 \times 15 \mathrm{~mL})$. The organic layer was dried over $\mathrm{MgSO}_{4}$, filtered and the solvent was removed under reduced pressure. The crude product was purified by column chromatography (eluant: ethyl acetate/hexane 1:60) to afford the corresponding enone. $0.150 \mathrm{~g}(67 \%)$; m.p. $82^{\circ} \mathrm{C}$; $[\alpha]_{\mathrm{D}}^{25}-54.0$ (c 1.0, $\mathrm{Cl}_{2} \mathrm{CH}_{2}$ ); IR (neat, cm $\left.{ }^{-1}\right) 3427(\mathrm{OH}), 1680$ $(\mathrm{CO}) ;{ }^{1} \mathrm{H}$ NMR $\left(\mathrm{CDCl}_{3}\right) \delta$ 7.01-6.90 $(\mathrm{dq}, 1 \mathrm{H}, \mathrm{HC}=\mathrm{C}, \mathrm{J}=7.0 \mathrm{~Hz}, \mathrm{~J}=14.7 \mathrm{~Hz}), 6.61(\mathrm{dq}, 1 \mathrm{H}, \mathrm{HC}=\mathrm{C}$, $\mathrm{J}=2.0 \mathrm{~Hz}, \mathrm{~J}=15.0 \mathrm{~Hz}), 3.13(\mathrm{~s}, 1 \mathrm{H}, \mathrm{OH}), 2.24(\mathrm{~d}, 1 \mathrm{H}, \mathrm{HCH}, \mathrm{J}=12.8 \mathrm{~Hz}), 1.87\left(\mathrm{~d}, 3 \mathrm{H}, \mathrm{CH}_{3}, 7.0 \mathrm{~Hz}\right)$, 1.80-1.61 (m, 2H, HCH), 1.40-1.14 (m, 3H, HCH), $1.08\left(\mathrm{~s}, 3 \mathrm{H}, \mathrm{CH}_{3}\right), 1.06-0.72(\mathrm{~m}, 1 \mathrm{H}, \mathrm{HCH})$, $0.85\left(\mathrm{~s}, 3 \mathrm{H}, \mathrm{CH}_{3}\right), 0.80\left(\mathrm{~s}, 3 \mathrm{H}, \mathrm{CH}_{3}\right) ;{ }^{13} \mathrm{C} \mathrm{NMR}\left(\mathrm{CDCl}_{3}\right) \delta 201.5,143.5,127.0,86.6,52.6,50.5$, 45.2, 41.1, 30.2, 26.6, 20.9, 20.4, 18.5, 10.6; Anal. calcd for $\mathrm{C}_{14} \mathrm{H}_{22} \mathrm{O}_{2}(222.36) \mathrm{C}, 75.62 ; \mathrm{H}, 9.99$. Found: C, 75.33; H, 10.07.

\section{General procedure for the Heck reaction}

A mixture of $\mathrm{Pd}_{2}(\mathrm{dba})_{3}(0.0022 \mathrm{~g}, 2.4 \mu \mathrm{mol}), \mathrm{PPh}_{3}(0.0052 \mathrm{~g}, 20 \mu \mathrm{mol})$ and $\mathrm{Et}_{3} \mathrm{~N}(0.28 \mathrm{~mL}, 2$ mmol) in $\mathrm{CH}_{3} \mathrm{CN}$ ( $4 \mathrm{~mL}$ ) was stirred for 30 minutes at room temperature. Then a solution of ketone $3(0.208 \mathrm{~g}, 1 \mathrm{mmol})$ and the corresponding iodobenzene $(3 \mathrm{mmol})$ was added and the mixture was stirred at $80^{\circ} \mathrm{C}$ for $1.5 \mathrm{~h}$. After this time, the reaction was allowed to warm to room temperature and then $1 \mathrm{~N} \mathrm{HCl}(5 \mathrm{~mL})$ was added and the mixture was extracted with $\mathrm{CH}_{2} \mathrm{Cl}_{2}(2 \times 15 \mathrm{~mL})$. The combined organic extracts were dried over $\mathrm{MgSO}_{4}$, filtered, and the solvent evaporated. The crude

\footnotetext{
${ }^{2}$ For alkylations of 1-alkoxyallenes, see: Hausherr, A.; Orschel, B.; Scherer, S.; Reissig, H.-U. Synthesis 2001, 1377-1385.
} 
product was purified by column chromatography (eluant: ethyl acetate/hexane 1:60) to afford the corresponding enone.

\section{(1R)-2-endo-cinnamoyl-1,7,7-trimethylbicyclo[2.2.1]heptan-2-ol. $\underline{7}$}

The title compound was prepared according to general procedure from iodobenzene $(0.34 \mathrm{~mL}, 3$ mmol) in $86 \%$ yield $\left(0.245\right.$ g). m.p. $133{ }^{\circ} \mathrm{C}$; $[\alpha]_{\mathrm{D}}^{25}-28.3\left(c 1.0, \mathrm{Cl}_{2} \mathrm{CH}_{2}\right)$; IR (neat, cm $\left.{ }^{-1}\right) 3462(\mathrm{OH})$, $1649(\mathrm{CO}) ;{ }^{1} \mathrm{H}$ NMR $\left(\mathrm{CDCl}_{3}\right) \delta$ 7.73-7.05 (m, 5H, arom.), $7.69(\mathrm{~d}, 1 \mathrm{H}, \mathrm{HC}=\mathrm{C}, \mathrm{J}=15.7 \mathrm{~Hz}), 7.28(\mathrm{~d}$, $1 \mathrm{H}, \mathrm{HC}=\mathrm{C}, \mathrm{J}=15.7 \mathrm{~Hz}), 3.06(\mathrm{~s}, 1 \mathrm{H}, \mathrm{OH}), 2.41(\mathrm{~d}, 1 \mathrm{H}, \mathrm{HCH}, \mathrm{J}=12.5 \mathrm{~Hz}), 1.98-1.11\left(\mathrm{~m}, 6 \mathrm{H}, 3 \mathrm{CH}_{2}\right)$, $1.19\left(\mathrm{~s}, 3 \mathrm{H}, \mathrm{CH}_{3}\right), 0.97\left(\mathrm{~s}, 3 \mathrm{H}, \mathrm{CH}_{3}\right), 0.88\left(\mathrm{~s}, 3 \mathrm{H}, \mathrm{CH}_{3}\right) ;{ }^{13} \mathrm{C} \mathrm{NMR}\left(\mathrm{CDCl}_{3}\right) \delta 219.0,134.9,130.6$, 129.0, 128.6, 87.3, 53.2, 50.8, 47.7, 43.6, 41.7, 30.6, 27.0, 21.1, 20.6, 19.6, 10.8; Anal. calcd for $\mathrm{C}_{19} \mathrm{H}_{24} \mathrm{O}_{2}(284.43) \mathrm{C}, 80.23 ; \mathrm{H}, 8.52$. Found: $\mathrm{C}, 80.36 ; \mathrm{H}, 8.66$.

\section{(1R)-2-endo-p-methylcinnamoyl-1,7,7-trimethylbicyclo[2.2.1]heptan-2-ol. $\underline{8}$}

The title compound was prepared according to general procedure from 4-iodotoluene $(0.65 \mathrm{~g}, 3$ mmol) in 98\% yield (0.292 g). m.p. $105-106^{\circ} \mathrm{C}$; $[\alpha]_{\mathrm{D}}^{25}-18.9\left(c \mathrm{c} 0.52, \mathrm{Cl}_{2} \mathrm{CH}_{2}\right)$; IR (neat, $\left.\mathrm{cm}^{-1}\right) 3467$ $(\mathrm{OH}), 1675(\mathrm{CO}) ;{ }^{1} \mathrm{H} \mathrm{NMR}\left(\mathrm{CDCl}_{3}\right) \delta 7.41(\mathrm{~d}, 1 \mathrm{H}, \mathrm{HC}=\mathrm{C}, \mathrm{J}=15.8 \mathrm{~Hz}), 7.21(\mathrm{~d}, 2 \mathrm{H}$, arom., $\mathrm{J}=8.1 \mathrm{~Hz}), 6.98(\mathrm{~d}, 1 \mathrm{H}, \mathrm{HC}=\mathrm{C}, \mathrm{J}=15.0 \mathrm{~Hz}), 6.92(\mathrm{~d}, 2 \mathrm{H}$, arom., $\mathrm{J}=8.0 \mathrm{~Hz}), 2.88(\mathrm{~s}, 1 \mathrm{H}, \mathrm{OH}), 2.14(\mathrm{~d}$, $1 \mathrm{H}, \mathrm{HCH}, \mathrm{J}=12.0 \mathrm{~Hz}), 2.12\left(\mathrm{~s}, 3 \mathrm{H}, \mathrm{CH}_{3}\right), 1.74-1.44(\mathrm{~m}, 3 \mathrm{H}, \mathrm{HCH}), 1.23-0.58(\mathrm{~m}, 3 \mathrm{H}, \mathrm{HCH}), 0.91$ (s, 3H, $\left.\mathrm{CH}_{3}\right), 0.69\left(\mathrm{~s}, 3 \mathrm{H}, \mathrm{CH}_{3}\right), 0.61\left(\mathrm{~s}, 3 \mathrm{H}, \mathrm{CH}_{3}\right) ;{ }^{13} \mathrm{C} \mathrm{NMR}\left(\mathrm{CDCl}_{3}\right) \delta 201.5,143.0,140.5,131.9$, 129.3, 128.3, 121.0, 87.0, 52.4, 50.6, 45.3, 41.3, 30.3, 26.5, 21.4, 20.9, 20.4, 10.7; Anal. calcd for $\mathrm{C}_{20} \mathrm{H}_{26} \mathrm{O}_{2}(298.46)$ C, 80.48; H, 8.80. Found: C, 80.54; H, 8.79.

\section{(1R)-2-endo-p-methoxycinnamoyl-1,7,7-trimethylbicyclo[2.2.1]heptan-2-ol. $\underline{9}$}

The title compound was prepared according to general procedure from 4-iodoanisole $(0.70 \mathrm{~g}, 3$ mmol) in $85 \%$ yield $(0.267 \mathrm{~g})$. m.p. $122-123{ }^{\circ} \mathrm{C}$; $[\alpha]_{\mathrm{D}}^{25}-16.6\left(c 1.0, \mathrm{Cl}_{2} \mathrm{CH}_{2}\right)$; IR (neat, $\left.\mathrm{cm}^{-1}\right) 3467$ $(\mathrm{OH}), 1664(\mathrm{CO}) ;{ }^{1} \mathrm{H}$ NMR $\left(\mathrm{CDCl}_{3}\right) \delta 7.66(\mathrm{~d}, 1 \mathrm{H}, \mathrm{HC}=\mathrm{C}, \mathrm{J}=15.8 \mathrm{~Hz}), 7.53(\mathrm{~d}, 2 \mathrm{H}$, arom., $\mathrm{J}=8.8 \mathrm{~Hz}), 7.15(\mathrm{~d}, 1 \mathrm{H}, \mathrm{HC}=\mathrm{C}, \mathrm{J}=15.4 \mathrm{~Hz}), 6.90(\mathrm{~d}, 2 \mathrm{H}, \operatorname{arom} ., \mathrm{J}=8.8 \mathrm{~Hz}), 3.84\left(\mathrm{~s}, 3 \mathrm{H}, \mathrm{CH}_{3}\right), 3.17$ (s, $1 \mathrm{H}, \mathrm{OH}), 2.38$ (d, 1H, HCH, J=12.8Hz), 2.00-1.72 (m, 4H, HCH), 1.49-1.24 (m, 4H, HCH), 1.17 (s, $\left.3 \mathrm{H}, \mathrm{CH}_{3}\right), 0.94\left(\mathrm{~s}, 3 \mathrm{H}, \mathrm{CH}_{3}\right), 0.87\left(\mathrm{~s}, 3 \mathrm{H}, \mathrm{CH}_{3}\right) ;{ }^{13} \mathrm{C} \mathrm{NMR}\left(\mathrm{CDCl}_{3}\right) \delta 201.8,161.5,143.1,130.2$, 127.5, 119.3, 114.3, 87.1, 55.4, 53.0, 50.6, 45.4, 41.5, 30.4, 26.9, 21.0, 20.4, 10.7; Anal. calcd for $\mathrm{C}_{20} \mathrm{H}_{26} \mathrm{O}_{3}$ (314.46) C, 76.39; H, 8.35. Found: C, 76.45; H, 8.29.

\section{(1R)-2-endo-p-chlorocinnamoyl-1,7,7-trimethylbicyclo[2.2.1]heptan-2-ol. $\underline{10}$}

The title compound was prepared according to general procedure from 1-chloro-4-iodobenzene (0.72 g, $3 \mathrm{mmol}$ ) in $92 \%$ yield (0.293 g). m.p. $126-127^{\circ} \mathrm{C}$; $[\alpha]_{\mathrm{D}}^{25}-19.6\left(c 1.0, \mathrm{Cl}_{2} \mathrm{CH}_{2}\right)$; IR (neat, cm ${ }^{-}$

1) $3457(\mathrm{OH}), 1680(\mathrm{CO})$; ${ }^{1} \mathrm{H}$ NMR $\left(\mathrm{CDCl}_{3}\right) \delta 7.58(\mathrm{~d}, 1 \mathrm{H}, \mathrm{HC}=\mathrm{C}, \mathrm{J}=15.8 \mathrm{~Hz}), 7.46(\mathrm{~d}, 2 \mathrm{H}$, arom., $\mathrm{J}=8.4 \mathrm{~Hz})$, ), 7.32 (d, 2H, arom., $\mathrm{J}=8.4 \mathrm{~Hz}), 7.22(\mathrm{~d}, 1 \mathrm{H}, \mathrm{HC}=\mathrm{C}, \mathrm{J}=15.9 \mathrm{~Hz}), 2.95(\mathrm{~s}, 1 \mathrm{H}, \mathrm{OH}), 2.39$ $(\mathrm{d}, 1 \mathrm{H}, \mathrm{HCH}, \mathrm{J}=12.8 \mathrm{~Hz}), 1.96-1.67(\mathrm{~m}, 2 \mathrm{H}, \mathrm{HCH}), 1.46-1.03(\mathrm{~m}, 4 \mathrm{H}, \mathrm{HCH}), 1.15\left(\mathrm{~s}, 3 \mathrm{H}, \mathrm{CH}_{3}\right)$, $0.94\left(\mathrm{~s}, 3 \mathrm{H}, \mathrm{CH}_{3}\right), 0.85\left(\mathrm{~s}, 3 \mathrm{H}, \mathrm{CH}_{3}\right) ;{ }^{13} \mathrm{C} \mathrm{NMR}\left(\mathrm{CDCl}_{3}\right) \delta 201.4,141.6,136.2,133.2,129.5,129.1$, 122.2 , 87.2, 52.8, 50.7, 45.4, 41.6, 30.4, 26.7, 20.9, 20.5, 10.8; Anal. calcd for $\mathrm{C}_{19} \mathrm{H}_{23} \mathrm{O}_{2} \mathrm{Cl}(318.87)$ C, 71.56; H, 7.28. Found: C, 70.97; H, 7.19. 


\section{Diels-Alder reactions with enone 3}

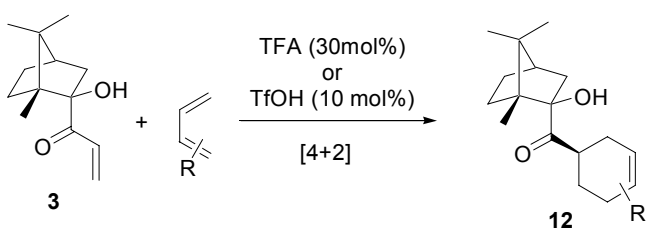

\section{General procedure A}

To a solution of $\alpha$ '-hydroxy vinyl ketone 3 (0.208 g, 1 mmol) in $\mathrm{CH}_{2} \mathrm{Cl}_{2}(4 \mathrm{~mL})$ cooled to $-25^{\circ} \mathrm{C}$ under a nitrogen atmosphere, the correspondig diene $(5 \mathrm{mmol})$ and trifluoroacetic acid $(0.06 \mathrm{~mL}, 0.3 \mathrm{mmol})$ were added dropwise. The mixture was stirred at $-25^{\circ} \mathrm{C}$ for 20 hours and then was quenched with a saturated aqueous solution of $\mathrm{NaHCO}_{3}(10 \mathrm{~mL})$. The resulting mixture was allowed to warm to room temperature, after which the layers were separated, and the aqueous layer was extracted with $\mathrm{CH}_{2} \mathrm{Cl}_{2}(2 \times 15 \mathrm{~mL})$. The combined organics were dried over $\mathrm{MgSO}_{4}$, and the solvent was removed under reduced pressure. Purification of the crude product 12 was effected by flash silica gel column chromatography using a 1:30 ethyl acetate/hexane mixture as the eluant.

\section{General procedure B}

To a solution of $\alpha$ '-hydroxy vinyl ketone $3(0.208 \mathrm{~g}, 1 \mathrm{mmol})$ in $\mathrm{CH}_{2} \mathrm{Cl}_{2}(4 \mathrm{~mL})$ cooled to $-78{ }^{\circ} \mathrm{C}$ under a nitrogen atmosphere, the correspondig diene $(5 \mathrm{mmol})$ and triflic acid $(0.01 \mathrm{~mL}, 0.1 \mathrm{mmol})$ were added dropwise. The mixture was stirred at $-78^{\circ} \mathrm{C}$ for 1 hour and then was quenched with a saturated aqueous solution of $\mathrm{NaHCO}_{3}(10 \mathrm{~mL})$. The resulting mixture was allowed to warm to room temperature, after which the layers were separated, and the aqueous layer was extracted with $\mathrm{CH}_{2} \mathrm{Cl}_{2}(2 \times 15 \mathrm{~mL})$. The combined organics were dried over $\mathrm{MgSO}_{4}$, and the solvent was removed under reduced pressure. Purification of the crude product 12 was effected by flash silica gel column chromatography using a 1:30 ethyl acetate/hexane mixture as the eluant.

(1R)-2-endo-[(1R)-4-methylcyclohex-3-en-1-ylcarbonyl]-1,7,7-trimethylbicyclo[2.2.1]heptan-2ol (12a). The title compound was prepared according to general procedure A from isoprene $(0.50$ $\mathrm{mL}, 5 \mathrm{mmol}) .0 .252 \mathrm{~g}$ (91\%); oil; $[\alpha]_{\mathrm{D}}^{25}-13.0$ (c 1.0, $\mathrm{Cl}_{2} \mathrm{CH}_{2}$ ); IR (neat, $\mathrm{cm}^{-1}$ ) 1696 (CO); ${ }^{1} \mathrm{H} \mathrm{NMR}$ $\left(\mathrm{CDCl}_{3}\right) \delta 5.36(\mathrm{~m}, 1 \mathrm{H}, \mathrm{HC}=\mathrm{C}), 3.00-2.95(\mathrm{~m}, 1 \mathrm{H}, \mathrm{CH}), 2.82(\mathrm{~s}, 1 \mathrm{H}, \mathrm{OH}), 2.24(\mathrm{~d}, 1 \mathrm{H}, \mathrm{HCH}$, $\mathrm{J}=13.2 \mathrm{~Hz}), 2.04-1.68(\mathrm{~m}, 6 \mathrm{H}, \mathrm{HCH}), 1.63\left(\mathrm{~s}, 3 \mathrm{H}, \mathrm{CH}_{3}\right), 1.46-0.93(\mathrm{~m}, 6 \mathrm{H}, \mathrm{HCH}), 1.08(\mathrm{~s}, 3 \mathrm{H}$, $\left.\mathrm{CH}_{3}\right), 0.89\left(\mathrm{~s}, 3 \mathrm{H}, \mathrm{CH}_{3}\right), 0.81\left(\mathrm{~s}, 3 \mathrm{H}, \mathrm{CH}_{3}\right) ;{ }^{13} \mathrm{C} \mathrm{NMR}\left(\mathrm{CDCl}_{3}\right) \delta 218.0,133.5,119.3,87.8,52.6$, $50.2,45.0,42.7,40.7,30.2,29.6,29.2,26.7,26.2,23.4,20.8,20.3,10.6$.

(1R)-2-endo-[(1R)-3,4-dimethylcyclohex-3-en-1-ylcarbonyl]-1,7,7-trimethylbicyclo[2.2.1]heptan-2-ol (12b). The title compound was prepared according to general procedure A or B from 2,3dimethyl-1,3-butadiene (0.56 mL, $5 \mathrm{mmol}) .0 .218 \mathrm{~g}(75 \%)$ (procedure A), $0.285 \mathrm{~g}(98 \%)$ (procedure B); m.p. $59{ }^{\circ} \mathrm{C}$; $[\alpha]_{\mathrm{D}}^{25}-3.0\left(c\right.$ 1.0, $\left.\mathrm{Cl}_{2} \mathrm{CH}_{2}\right)$; IR (neat, $\left.\mathrm{cm}^{-1}\right) 1696(\mathrm{CO}) ;{ }^{1} \mathrm{H} \mathrm{NMR}\left(\mathrm{CDCl}_{3}\right)$ d 3.32-2.95 (m, 1H, CH), $2.85(\mathrm{~s}, 1 \mathrm{H}, \mathrm{OH}), 2.25$ (d, 1H, HCH, J=13.2Hz), 2.15-1.64 (m, 8H, HCH), $1.59\left(\mathrm{~s}, 6 \mathrm{H}, 2 \mathrm{CH}_{3}\right), 1.47-0.57(\mathrm{~m}, 4 \mathrm{H}, \mathrm{CH}), 1.09\left(\mathrm{~s}, 3 \mathrm{H}, \mathrm{CH}_{3}\right), 0.89\left(\mathrm{~s}, 3 \mathrm{H}, \mathrm{CH}_{3}\right), 0.82(\mathrm{~s}, 3 \mathrm{H}$, $\left.\mathrm{CH}_{3}\right) ;{ }^{13} \mathrm{C} \mathrm{NMR}\left(\mathrm{CDCl}_{3}\right) \delta 218.0,125.2,124.0,87.7,52.7,50.2,45.0,43.8,40.7,35.2,31.3,30.2$, 26.8, 26.4, 20.8, 20.3, 19.0, 18.9, 10.6; Anal. calcd for $\mathrm{C}_{19} \mathrm{H}_{30} \mathrm{O}_{2}(290.49) \mathrm{C}, 78.55$; $\mathrm{H}, 10.43$. Found: C, 79.01; H, 10.25.

(1R)-2-endo-[(1R, 2R, 4R)-bicyclo[2.2.1]hept-5-en-2-ylcarbonyl]-1,7,7-trimethylbicyclo[2.2.1]heptan-2-ol (12c). The title compound was prepared according to general procedure B from 
cyclopentadiene $(0.40 \mathrm{~mL}, 5 \mathrm{mmol})$ in 1 h. $0.269 \mathrm{~g}(98 \%)$; m.p. $102-103{ }^{\circ} \mathrm{C} ;[\alpha]_{\mathrm{D}}^{25}+71.0$ (c 1.0, $\left.\mathrm{Cl}_{2} \mathrm{CH}_{2}\right)$; IR (neat, $\left.\mathrm{cm}^{-1}\right) 1701(\mathrm{CO}) ;{ }^{1} \mathrm{H} \mathrm{NMR}\left(\mathrm{CDCl}_{3}\right) \delta 6.23(\mathrm{dd}, 1 \mathrm{H}, \mathrm{HC}=\mathrm{C}, \mathrm{J}=2.9 \mathrm{~Hz}$, $\left.\mathrm{J}^{\prime}=5.5 \mathrm{~Hz}\right), 5.69(\mathrm{dd}, 1 \mathrm{H}, \mathrm{HC}=\mathrm{C}, \mathrm{J}=2.9 \mathrm{~Hz}, \mathrm{~J}=5.5 \mathrm{~Hz}), 3.45(\mathrm{~m}, 1 \mathrm{H}, \mathrm{CH}), 3.14(\mathrm{~m}, 1 \mathrm{H}, \mathrm{CH}), 3.10(\mathrm{~s}$, 1H, OH), $2.92(\mathrm{~m}, 1 \mathrm{H}, \mathrm{CH}), 2.32(\mathrm{~d}, 1 \mathrm{H}, \mathrm{HCH}, \mathrm{J}=13.6 \mathrm{~Hz}), 2.07-1.97$ (m, 1H, HCH), 1.88-1.70 (m, $3 \mathrm{H}, \mathrm{HCH}), 1.46-1.09(\mathrm{~m}, 5 \mathrm{H}, \mathrm{HCH}), 1.07\left(\mathrm{~s}, 3 \mathrm{H}, \mathrm{CH}_{3}\right), 1.03-0.80(\mathrm{~m}, 1 \mathrm{H}, \mathrm{HCH}), 0.82\left(\mathrm{~s}, 3 \mathrm{H}, \mathrm{CH}_{3}\right)$, $0.81\left(\mathrm{~s}, 3 \mathrm{H}, \mathrm{CH}_{3}\right) ;{ }^{13} \mathrm{C} \mathrm{NMR}\left(\mathrm{CDCl}_{3}\right) \delta 216.6,137.6,130.8,86.0,53.3,50.5,49.7,47.6,46.8,45.1$, 43.3, 40.2, 30.6, 29.9, 27.2, 20.7, 20.1, 9.9; Anal. calcd for $\mathrm{C}_{18} \mathrm{H}_{26} \mathrm{O}_{2}$ (274.44) C, 78.77; H, 9.57. Found: C, 78.59; H, 9.56.

(1R)-2-endo-[(1R，2R，4R)-bicyclo[2.2.1]oct-5-en-2-ylcarbonyl]-1,7,7-trimethylbicyclo[2.2.1]heptan-2-ol (12d). The title compound was prepared according to general procedure $\mathrm{B}$ from 1,3cyclohexadiene (0.48 mL, $5 \mathrm{mmol}) .0 .245 \mathrm{~g}$ (85\%); m.p. 83-84 ${ }^{\circ} \mathrm{C} ;[\alpha]_{\mathrm{D}}^{25}-10.0\left(c 1.0, \mathrm{Cl}_{2} \mathrm{CH}_{2}\right)$; IR (neat, $\left.\mathrm{cm}^{-1}\right) 1685(\mathrm{CO}) ;{ }^{1} \mathrm{H}$ NMR $\left(\mathrm{CDCl}_{3}\right) \delta 6.35(\mathrm{t}, 1 \mathrm{H}, \mathrm{HC}=\mathrm{C}, \mathrm{J}=8.0 \mathrm{~Hz}), 6.04(\mathrm{t}, 1 \mathrm{H}, \mathrm{HC}=\mathrm{C}$, $\mathrm{J}=7.0 \mathrm{~Hz}), 3.13(\mathrm{~m}, 1 \mathrm{H}, \mathrm{CH}), 3.10(\mathrm{~s}, 1 \mathrm{H}, \mathrm{OH}), 2.68-2.64(\mathrm{~m}, 2 \mathrm{H}, \mathrm{CH}), 2.15(\mathrm{~d}, 1 \mathrm{H}, \mathrm{HCH}$, $\mathrm{J}=13.6 \mathrm{~Hz}), 2.05-1.95(\mathrm{~m}, 1 \mathrm{H}, \mathrm{HCH}), 1.87-1.42(\mathrm{~m}, 6 \mathrm{H}, \mathrm{HCH}), 1.38-1.12(\mathrm{~m}, 4 \mathrm{H}, \mathrm{HCH}), 1.08(\mathrm{~s}$, $\left.3 \mathrm{H}, \mathrm{CH}_{3}\right), 1.00-0.80(\mathrm{~m}, 1 \mathrm{H}, \mathrm{HCH}), 0.81\left(\mathrm{~s}, 6 \mathrm{H}, 2 \mathrm{CH}_{3}\right) ;{ }^{13} \mathrm{C} \mathrm{NMR}\left(\mathrm{CDCl}_{3}\right) \delta 216.6,134.9,130.3$, 86.1, 53.3, 49.8, 45.5, 45.1, 40.5, 32.2, 31.2, 29.8, 29.5, 27.2, 26.6, 23.5, 20.7, 20.1, 10.0; Anal. calcd for $\mathrm{C}_{19} \mathrm{H}_{28} \mathrm{O}_{2}(288.47) \mathrm{C}, 79.10 ; \mathrm{H}, 9.80$. Found: C, 79.18; H, 9.76.

\section{Diels-Alder reactions with enone 6-10}

To a solution of the corresponding $\alpha$ '-hydroxy vinyl ketone (1 mmol) in $\mathrm{CH}_{2} \mathrm{Cl}_{2}(4 \mathrm{~mL})$ cooled to $78{ }^{\circ} \mathrm{C}$ under a nitrogen atmosphere, the correspondig diene $(5 \mathrm{mmol})$ and trific acid $(0.01 \mathrm{~mL}, 0.1$ $\mathrm{mmol})$ or $(0.03 \mathrm{~mL}, 0.3 \mathrm{mmol})$ were added dropwise. The mixture was stirred at $-78{ }^{\circ} \mathrm{C}$ for 0.5 to 3 hours and then was quenched with a saturated aqueous solution of $\mathrm{NaHCO}_{3}(10 \mathrm{~mL})$. The resulting mixture was allowed to warm to room temperature, after which the layers were separated, and the aqueous layer was extracted with $\mathrm{CH}_{2} \mathrm{Cl}_{2}(2 \times 15 \mathrm{~mL})$. The combined organics were dried over $\mathrm{MgSO}_{4}$, and the solvent was removed under reduced pressure. Purification of the crude product was effected by flash silica gel column chromatography using a 1:30 ethyl acetate/hexane mixture as the eluant.

(1R)-2-endo-[(1S， 6S)-4,6-dimethylcyclohex-3-en-1-ylcarbonyl]-1,7,7-trimethylbicyclo[2.2.1]heptan-2-ol. (13)

The title compound was prepared according to general procedure from $6(0.222 \mathrm{~g}, 1 \mathrm{mmol})$, isoprene $(0.50 \mathrm{~mL}, 5 \mathrm{mmol})$ and TfOH $(0.1 \mathrm{mmol})$ in $0.5 \mathrm{~h} .0 .270 \mathrm{~g}(93 \%)$; oil; $[\alpha]_{\mathrm{D}}^{25}+49.0\left(c 1.0, \mathrm{Cl}_{2} \mathrm{CH}_{2}\right)$; IR (neat, $\left.\mathrm{cm}^{-1}\right) 3534(\mathrm{OH}), 1691(\mathrm{CO}) ;{ }^{1} \mathrm{H}$ NMR $\left(\mathrm{CDCl}_{3}\right) \delta 5.31(\mathrm{~m}, 1 \mathrm{H}, \mathrm{HC}=\mathrm{C}), 2.93-2.80(\mathrm{~m}, 1 \mathrm{H}$, $\mathrm{CH}), 2.34-2.19(\mathrm{~m}, 1 \mathrm{H}, \mathrm{CH}), 2.32(\mathrm{~s}, 1 \mathrm{H}, \mathrm{OH}), 2.13-1.82(\mathrm{~m}, 3 \mathrm{H}, \mathrm{HCH}), 1.80-1.65(\mathrm{~m}, 4 \mathrm{H}, \mathrm{HCH})$, $1.60\left(\mathrm{~s}, 3 \mathrm{H}, \mathrm{CH}_{3}\right), 1.41-0.88(\mathrm{~m}, 4 \mathrm{H}, \mathrm{HCH}), 1.09\left(\mathrm{~s}, 3 \mathrm{H}, \mathrm{CH}_{3}\right), 0.93\left(\mathrm{~s}, 3 \mathrm{H}, \mathrm{CH}_{3}\right), 0.85\left(\mathrm{~s}, 3 \mathrm{H}, \mathrm{CH}_{3}\right)$, $0.80\left(\mathrm{~s}, 3 \mathrm{H}, \mathrm{CH}_{3}\right) ;{ }^{13} \mathrm{C} \mathrm{NMR}\left(\mathrm{CDCl}_{3}\right) \delta 217.4,133.6,119.1,87.8,52.3,50.7,48.0,44.8,41.1,38.9$, $32.3,30.8,29.9,26.4,23.1,20.9,20.5,20.2,10.8$.

(1R)-2-endo-[(1S, [2.2.1]heptan-2-ol. (14)

The title compound was prepared according to general procedure from $6(0.222 \mathrm{~g}, 1 \mathrm{mmol}), 2,3-$ dimethyl-1,3-butadiene $(0.56 \mathrm{~mL}, 5 \mathrm{mmol})$ and TfOH $(0.1 \mathrm{mmol})$ in $1 \mathrm{~h} .0 .289 \mathrm{~g}(95 \%)$; oil; $[\alpha]_{\mathrm{D}}^{25}$ +54.0 (c 1.0, $\left.\mathrm{Cl}_{2} \mathrm{CH}_{2}\right)$; IR (neat, $\left.\mathrm{cm}^{-1}\right) 3519(\mathrm{OH}), 1611(\mathrm{CO}) ;{ }^{1} \mathrm{H} \mathrm{NMR}\left(\mathrm{CDCl}_{3}\right) \delta$ 2.97-2.84 (m, $1 \mathrm{H}, \mathrm{CH}), 2.40(\mathrm{~s}, 1 \mathrm{H}, \mathrm{OH}), 2.29(\mathrm{~d}, 1 \mathrm{H}, \mathrm{HCH}, \mathrm{J}=12.5 \mathrm{~Hz}), 2.00-1.59(\mathrm{~m}, 8 \mathrm{H}, \mathrm{CH}), 1.55(\mathrm{~s}, 6 \mathrm{H}, 2$ $\left.\mathrm{CH}_{3}\right), 1.39-0.88(\mathrm{~m}, 4 \mathrm{H}, \mathrm{CH}), 1.08\left(\mathrm{~s}, 3 \mathrm{H}, \mathrm{CH}_{3}\right), 0.92\left(\mathrm{~s}, 3 \mathrm{H}, \mathrm{CH}_{3}\right), 0.82\left(\mathrm{~d}, 3 \mathrm{H}, \mathrm{CH}_{3}, \mathrm{~J}=5.9 \mathrm{~Hz}\right)$, 
$0.80\left(\mathrm{~s}, 3 \mathrm{H}, \mathrm{CH}_{3}\right) ;{ }^{13} \mathrm{C} \mathrm{NMR}\left(\mathrm{CDCl}_{3}\right) \delta 217.4,125.2,123.8,87.7,52.3,50.6,50.0,44.9$, $41.0,40.6,37.0,32.6,29.9,26.5,20.8,20.5,20.0,18.7,18.7,10.9$.

(1R)-2-endo-[(1R, 2R， 3S， 4S)-3-methylbicyclo[2.2.1]hept-5-en-2-ylcarbonyl]-1,7,7trimethylbicyclo[2.2.1] heptan-2-ol. (15)

The title compound was prepared according to general procedure from $\mathbf{6}(0.222 \mathrm{~g}, 1 \mathrm{mmol})$, cyclopentadiene $(0.40 \mathrm{~mL}, 5 \mathrm{mmol})$ and TFA $(0.3 \mathrm{mmol})$ at $-25^{\circ} \mathrm{C}$ for $1 \mathrm{~h} .0 .274 \mathrm{~g}(95 \%)$; m.p. 63$64{ }^{\circ} \mathrm{C} ;[\alpha]_{\mathrm{D}}^{25}+101.0\left(c\right.$ 1.0, $\left.\mathrm{Cl}_{2} \mathrm{CH}_{2}\right)$; IR (neat, $\left.\mathrm{cm}^{-1}\right) 3508,(\mathrm{OH}), 1685(\mathrm{CO}) ;{ }^{1} \mathrm{H}$ NMR $\left(\mathrm{CDCl}_{3}\right) \delta$ $6.32\left(\mathrm{dd}, 1 \mathrm{H}, \mathrm{HC}=\mathrm{C}, \mathrm{J}=3.3 \mathrm{~Hz}, \mathrm{~J}^{\prime}=5.9 \mathrm{~Hz}\right), 5.69(\mathrm{dd}, 1 \mathrm{H}, \mathrm{HC}=\mathrm{C}, \mathrm{J}=2.9 \mathrm{~Hz}, \mathrm{~J} '=5.9 \mathrm{~Hz}), 3.10(\mathrm{~m}, 1 \mathrm{H}$, $\mathrm{CH}), 2.98(\mathrm{~m}, 1 \mathrm{H}, \mathrm{CH}), 2.95(\mathrm{~s}, 1 \mathrm{H}, \mathrm{OH}), 2.49(\mathrm{~m}, 1 \mathrm{H}, \mathrm{CH}), 2.33(\mathrm{~d}, 1 \mathrm{H}, \mathrm{HCH}, \mathrm{J}=13.9 \mathrm{~Hz}), 2.06-$ $1.95(\mathrm{~m}, 1 \mathrm{H}, \mathrm{HCH}), 1.88-1.70(\mathrm{~m}, 3 \mathrm{H}, \mathrm{HCH}), 1.70-1.19(\mathrm{~m}, 4 \mathrm{H}, \mathrm{HCH}), 1.10\left(\mathrm{~d}, 3 \mathrm{H}, \mathrm{CH}_{3}\right.$, $\mathrm{J}=8.1 \mathrm{~Hz}), 1.08\left(\mathrm{~s}, 3 \mathrm{H}, \mathrm{CH}_{3}\right), 1.04-0.83(\mathrm{~m}, 1 \mathrm{H}, \mathrm{HCH}), 0.89\left(\mathrm{~s}, 3 \mathrm{H}, \mathrm{CH}_{3}\right), 0.81\left(\mathrm{~s}, 3 \mathrm{H}, \mathrm{CH}_{3}\right) ;{ }^{13} \mathrm{C}$ NMR $\left(\mathrm{CDCl}_{3}\right) \delta 216.0,138.3,131.2,85.9,55.7,53.4,50.1,49.8,48.2,46.8,47.1,45.0,40.0,38.0$, 29.8, 27.0, 20.7, 20.6, 20.1, 10.0; Anal. calcd for $\mathrm{C}_{19} \mathrm{H}_{28} \mathrm{O}_{2}$ (288.47) C, 79.10; H, 9.80. Found: C, $79.23 ; \mathrm{H}, 9.59$.

(1R)-2-endo-[(1R，2R，3S，4S)-3-methylbicyclo[2.2.1]oct-5-en-2-ylcarbonyl]-1,7,7-trimethylbicyclo[2.2.1]heptan-2-ol. (16)

The title compound was prepared according to general procedure from $\mathbf{6}(0.222 \mathrm{~g}, 1 \mathrm{mmol}), 1,3-$ cyclohexadiene $(0.48 \mathrm{~mL}, 5 \mathrm{mmol})$ and TfOH $(0.1 \mathrm{mmol})$ in $1.5 \mathrm{~h} .0 .272 \mathrm{~g}(90 \%)$; oil; $[\alpha]_{\mathrm{D}}^{25}+12.0$ (c 1.0, $\left.\mathrm{Cl}_{2} \mathrm{CH}_{2}\right)$; IR (neat, $\left.\mathrm{cm}^{-1}\right) 1685(\mathrm{CO}) ;{ }^{1} \mathrm{H}$ NMR $\left(\mathrm{CDCl}_{3}\right) \delta 6.46(\mathrm{t}, 1 \mathrm{H}, \mathrm{HC}=\mathrm{C}, \mathrm{J}=8.1 \mathrm{~Hz}), 5.87$ $(\mathrm{t}, 1 \mathrm{H}, \mathrm{HC}=\mathrm{C}, \mathrm{J}=7.7 \mathrm{~Hz}), 2.95(\mathrm{~s}, 1 \mathrm{H}, \mathrm{OH}), 2.63(\mathrm{~m}, 3 \mathrm{H}, \mathrm{HCH}), 2.30-2.26(\mathrm{~m}, 1 \mathrm{H}, \mathrm{HCH}), 2.17(\mathrm{~d}$, $1 \mathrm{H}, \mathrm{HCH}, \mathrm{J}=13.9 \mathrm{~Hz}), 2.10-1.63(\mathrm{~m}, 4 \mathrm{H}, \mathrm{HCH}), 1.57-0.80(\mathrm{~m}, 6 \mathrm{H}, \mathrm{HCH}), 1.07\left(\mathrm{~s}, 3 \mathrm{H}, \mathrm{CH}_{3}\right), 1.00$ $\left(\mathrm{d}, 3 \mathrm{H}, \mathrm{CH}_{3}, \mathrm{~J}=7.0 \mathrm{~Hz}\right), 0.86\left(\mathrm{~s}, 3 \mathrm{H}, \mathrm{CH}_{3}\right), 0.80\left(\mathrm{~s}, 3 \mathrm{H}, \mathrm{CH}_{3}\right) ;{ }^{13} \mathrm{C} \mathrm{NMR}\left(\mathrm{CDCl}_{3}\right) \delta 216.1,137.0$, 129.0, 85.8, 54.5, 53.5, 49.9, 45.0, 40.4, 36.2, 34.2, 33.2, 29.9, 27.1, 26.7, 20.6, 20.1, 19.3, 17.7, 10.0 .

(1R)-2-endo-[(1S, 6S)-4-methyl-6-phenylcyclohex-3-en-1-ylcarbonyl]-1,7,7-trimethylbicyclo[2.2.1] heptan-2-ol. (17)

The title compound was prepared according to general procedure from $7(0.284 \mathrm{~g}, 1 \mathrm{mmol})$, isoprene $(0.50 \mathrm{ml}, 5 \mathrm{mmol})$ and TfOH $(0.3 \mathrm{mmol})$ in $2 \mathrm{~h} .0 .300 \mathrm{~g}(85 \%)$; oil; $[\alpha]_{\mathrm{D}}^{25}+12.6$ (c 2.6, $\left.\mathrm{Cl}_{2} \mathrm{CH}_{2}\right)$; IR (neat, $\left.\mathrm{cm}^{-1}\right) 3550(\mathrm{OH}), 1696(\mathrm{CO}) ;{ }^{1} \mathrm{H}$ NMR $\left(\mathrm{CDCl}_{3}\right) \delta 7.25-7.04(\mathrm{~m}, 5 \mathrm{H}$, arom. $), 5.40(\mathrm{~m}, 1 \mathrm{H}$, $\mathrm{HC}=\mathrm{C}), 3.52-3.41(\mathrm{~m}, 1 \mathrm{H}, \mathrm{HCPh}), 3.06-2.92(\mathrm{~m}, 2 \mathrm{H}, \mathrm{HCCO}), 2.60-2.20(\mathrm{~m}, 2 \mathrm{H}, \mathrm{HCH}), 1.90(\mathrm{~d}$, $1 \mathrm{H}, \mathrm{HCH}, \mathrm{J}=13.9 \mathrm{~Hz}), 1.70\left(\mathrm{~s}, 3 \mathrm{H}, \mathrm{CH}_{3}\right), 1.76-1.36(\mathrm{~m}, 3 \mathrm{H}, \mathrm{HCH}), 1.19-0.52(\mathrm{~m}, 5 \mathrm{H}, \mathrm{HCH}), 0.88$ $\left(\mathrm{s}, 3 \mathrm{H}, \mathrm{CH}_{3}\right), 0.81\left(\mathrm{~s}, 3 \mathrm{H}, \mathrm{CH}_{3}\right), 0.67\left(\mathrm{~s}, 3 \mathrm{H}, \mathrm{CH}_{3}\right), 0.49(\mathrm{~s}, 1 \mathrm{H}, \mathrm{OH}) ;{ }^{13} \mathrm{C} \mathrm{NMR}\left(\mathrm{CDCl}_{3}\right) \delta 216.4$, $143.5,133.2,128.3,128.3,126.9,119.3,87.8,51.9,51.0,47.8,44.8,44.2,41.8,36.2,30.4,30.2$, 26.0, 23.3, 20.9, 20.6, 11.2 .

(1R)-2-endo-[(1R, 2S, 3S, 4S)-3-phenylbicyclo[2.2.1]hept-5-en-2-ylcarbonyl]-1,7,7-trimethylbicyclo[2.2.1] heptan-2-ol. (18)

The title compound was prepared according to general procedure from $7(0.284 \mathrm{~g}, 1 \mathrm{mmol})$, cyclopentadiene $(0.40 \mathrm{~mL}, 5 \mathrm{mmol})$ and $\mathrm{TfOH}(0.3 \mathrm{mmol})$ in 3 h. $0.305 \mathrm{~g}(87 \%)$; oil; $[\alpha]_{\mathrm{D}}^{25}+129.0$ (c 1.0, $\left.\mathrm{Cl}_{2} \mathrm{CH}_{2}\right)$; IR (neat, $\left.\mathrm{cm}^{-1}\right) 3514(\mathrm{OH}), 1680(\mathrm{CO}) ;{ }^{1} \mathrm{H}$ NMR $\left(\mathrm{CDCl}_{3}\right) \delta$ 7.31-7.16 (m, 5H, arom.), $6.48(\mathrm{dd}, 1 \mathrm{H}, \mathrm{HC}=\mathrm{C}, \mathrm{J}=3.3 \mathrm{~Hz}, \mathrm{~J} '=5.5 \mathrm{~Hz}), 5.87(\mathrm{dd}, 1 \mathrm{H}, \mathrm{HC}=\mathrm{C}, \mathrm{J}=2.9 \mathrm{~Hz}, \mathrm{~J} '=5.5 \mathrm{~Hz}), 3.65$ $\left(\mathrm{dd}, 1 \mathrm{H}, \mathrm{HCPh}, \mathrm{J}=3.3 \mathrm{~Hz}, \mathrm{~J}^{\prime}=5.5 \mathrm{~Hz}\right), 3.28(\mathrm{~m}, 1 \mathrm{H}, \mathrm{CH}), 3.02(\mathrm{dd}, 1 \mathrm{H}, \mathrm{HCCO}, \mathrm{J}=1.5 \mathrm{~Hz}, \mathrm{~J} '=5.5 \mathrm{~Hz})$, $2.89(\mathrm{~m}, 1 \mathrm{H}, \mathrm{CH}), 2.85(\mathrm{~s}, 1 \mathrm{H}, \mathrm{OH}), 2.33(\mathrm{~d}, 1 \mathrm{H}, \mathrm{HCH}, \mathrm{J}=13.9 \mathrm{~Hz}), 2.06-1.68(\mathrm{~m}, 3 \mathrm{H}, \mathrm{HCH}), 1.66-$ $1.54(\mathrm{~m}, 2 \mathrm{H}, \mathrm{HCH}), 1.25-0.63(\mathrm{~m}, 3 \mathrm{H}, \mathrm{HCH}), 1.06\left(\mathrm{~s}, 3 \mathrm{H}, \mathrm{CH}_{3}\right), 0.85\left(\mathrm{~s}, 3 \mathrm{H}, \mathrm{CH}_{3}\right), 0.75(\mathrm{~s}, 3 \mathrm{H}$, 
$\left.\mathrm{CH}_{3}\right) ;{ }^{13} \mathrm{C} \mathrm{NMR}\left(\mathrm{CDCl}_{3}\right) \delta 215.1,143.6,138.7,132.5,128.3,127.2,126.1,86.1,53.9$, 53.3, 51.0, 49.9, 48.8, 47.9, 44.9, 40.3, 29.4, 27.0, 20.6, 20.0, 10.1.

(1R)-2-endo-[(1S, 6S)-3,4 -dimethyl-6-p-methylphenylcyclohex-3-en-1-ylcarbonyl]-1,7,7trimethylbicyclo[2.2.1] heptan-2-ol. (19)

The title compound was prepared according to general procedure from $8(0.298 \mathrm{~g}, 1 \mathrm{mmol}), 2,3-$ dimethyl-1,3-butadiene $(0.56 \mathrm{~mL}, 5 \mathrm{mmol})$ and TfOH $(0.3 \mathrm{mmol})$ in $1.5 \mathrm{~h} .0 .342 \mathrm{~g}(90 \%)$; oil; $[\alpha]_{\mathrm{D}}^{25}$ + 32.1 (c 1.45, $\left.\mathrm{Cl}_{2} \mathrm{CH}_{2}\right)$; IR (neat, $\left.\mathrm{cm}^{-1}\right) 3549(\mathrm{OH}), 1701(\mathrm{CO}) ;{ }^{1} \mathrm{H} \mathrm{NMR}\left(\mathrm{CDCl}_{3}\right) \delta$ 7.18-7.01 (m, 4H, arom.), 3.49 (m, 1H, HCAr), 2.90 (m, 1H, HCCO), 2.50-1.91 (m, 3H, HCH), 2.22 (s, 3H, $\left.\mathrm{CH}_{3} \mathrm{C}_{6} \mathrm{H}_{4}\right), 1.60\left(\mathrm{~s}, 6 \mathrm{H}, 2 \mathrm{CH}_{3}\right), 1.55-1.40(\mathrm{~m}, 3 \mathrm{H}, \mathrm{HCH}), 1.38-0.68(\mathrm{~m}, 5 \mathrm{H}, \mathrm{HCH}), 0.89(\mathrm{~s}, 3 \mathrm{H}$, $\left.\mathrm{CH}_{3}\right), 0.82\left(\mathrm{~s}, 3 \mathrm{H}, \mathrm{CH}_{3}\right), 0.68\left(\mathrm{~s}, 3 \mathrm{H}, \mathrm{CH}_{3}\right), 0.56(\mathrm{~s}, 1 \mathrm{H}, \mathrm{OH}) ;{ }^{13} \mathrm{C} \mathrm{NMR}\left(\mathrm{CDCl}_{3}\right) \delta 216.4,140.4$, 136.4, 128.9, 128.3, 128.1, 124.9, 124.0, 87.8, 51.9, 51.0, 48.8, 44.9, 44.2, 41.8, 37.9, 36.6, 30.3, 26.0, 21.0, 21.0, 20.6, 18.8, 18.7, 11.3.

(1R)-2-endo-[(1R, 2S, 3S, 4S)-3-p-methoxyphenylbicyclo[2.2.1]hept-5-en-2-ylcarbonyl]-1,7,7trimethyl-bicyclo[2.2.1] heptan-2-ol. (20)

The title compound was prepared according to general procedure from 9 (0.314 g, $1 \mathrm{mmol})$, cyclopentadiene $(0.40 \mathrm{~mL}, 5 \mathrm{mmol})$ and TfOH $(0.3 \mathrm{mmol})$ in 1 h. $0.320 \mathrm{~g}(84 \%)$; oil; $[\alpha]_{\mathrm{D}}^{25}+123.5$ (c $\left.0.9, \mathrm{Cl}_{2} \mathrm{CH}_{2}\right)$; IR (neat, $\left.\mathrm{cm}^{-1}\right) 3503(\mathrm{OH}), 1685(\mathrm{CO}) ;{ }^{1} \mathrm{H} \mathrm{NMR}\left(\mathrm{CDCl}_{3}\right) \delta 7.12(\mathrm{~d}, 2 \mathrm{H}, \mathrm{J}=8.8 \mathrm{~Hz}$, arom.), 6.80 (d, $2 \mathrm{H}, \mathrm{J}=8.8 \mathrm{~Hz}$, arom.), $6.44(\mathrm{dd}, 1 \mathrm{H}, \mathrm{HC}=\mathrm{C}, \mathrm{J}=2.2 \mathrm{~Hz}, \mathrm{~J}=6.6 \mathrm{~Hz}), 5.82$ (dd, $1 \mathrm{H}$, $\left.\mathrm{HC}=\mathrm{C}, \mathrm{J}=3.3 \mathrm{~Hz}, \mathrm{~J}^{\prime}=5.9 \mathrm{~Hz}\right), 3.71\left(\mathrm{~s}, 3 \mathrm{H}, \mathrm{CH}_{3} \mathrm{O}\right), 3.54(\mathrm{dd}, 1 \mathrm{H}, \mathrm{HCAr}, \mathrm{J}=2.9 \mathrm{~Hz}, \mathrm{~J}=5.5 \mathrm{~Hz}), 3.22(\mathrm{~m}$, $1 \mathrm{H}, \mathrm{CH}), 2.93(\mathrm{~m}, 1 \mathrm{H}, \mathrm{HCCO}), 2.81(\mathrm{~s}, 1 \mathrm{H}, \mathrm{OH}), 2.76(\mathrm{~m}, 1 \mathrm{H}, \mathrm{CH}), 2.26(\mathrm{~d}, 1 \mathrm{H}, \mathrm{HCH}, \mathrm{J}=13.9 \mathrm{~Hz})$, 2.12-1.84 (m, 3H, HCH), 1.79-1.47 (m, 3H, HCH), 1.20-1.04 (m, 1H, HCH), $1.00\left(\mathrm{~s}, 3 \mathrm{H}, \mathrm{CH}_{3}\right)$, 0.85-0.59 (m, 1H, HCH), $0.78\left(\mathrm{~s}, 3 \mathrm{H}, \mathrm{CH}_{3}\right), 0.69\left(\mathrm{~s}, 3 \mathrm{H}, \mathrm{CH}_{3}\right) ;{ }^{13} \mathrm{C}$ NMR $\left(\mathrm{CDCl}_{3}\right) \delta 215.1,157.9$, 138.9, 132.5, 128.2, 113.8, 86.2, 55.2, 54.3, 53.5 51.3, 50.0, 48.3, 48.0, 45.0, 40.3, 29.5, 27.1, 20.7, $20.1,10.1$.

(1R)-2-endo-[(1S, 6S)-3,4 -dimethyl-6-p-chlorophenylcyclohex-3-en-1-ylcarbonyl]-1,7,7-trimethylbicyclo[2.2.1] heptan-2-ol. (21)

The title compound was prepared according to general procedure from $\mathbf{1 0}(0.319 \mathrm{~g}, 1 \mathrm{mmol}), 2,3-$ dimethyl-1,3-butadiene $(0.56 \mathrm{~mL}, 5 \mathrm{mmol})$ and $\mathrm{TfOH}(0.1 \mathrm{mmol})$ in $1 \mathrm{~h} .0 .381 \mathrm{~g}(95 \%)$; oil; $[\alpha]_{\mathrm{D}}^{25}$ +28.4 (c 2.6, $\left.\mathrm{Cl}_{2} \mathrm{CH}_{2}\right)$; IR (neat, $\left.\mathrm{cm}^{-1}\right) 3565(\mathrm{OH}), 1691(\mathrm{CO}) ;{ }^{1} \mathrm{H} \mathrm{NMR}\left(\mathrm{CDCl}_{3}\right) \delta$ 7.25-7.04 (m, $4 \mathrm{H}$, arom.), 3.47 (m, 1H, HCAr, J=5.5Hz, J'=11.0Hz), $3.05(\mathrm{~m}, 1 \mathrm{H}, \mathrm{HCCO}, \mathrm{J}=5.1 \mathrm{~Hz}, \mathrm{~J}=11.4 \mathrm{~Hz})$, 2.43-1.97 (m, 4H, HCH), $1.89(\mathrm{~d}, 1 \mathrm{H}, \mathrm{HCH}, \mathrm{J}=13.2 \mathrm{~Hz}), 1.59\left(\mathrm{~s}, 6 \mathrm{H}, 2 \mathrm{CH}_{3}\right), 1.55-1.40(\mathrm{~m}, 3 \mathrm{H}$, $\mathrm{HCH}), 1.35-0.60(\mathrm{~m}, 4 \mathrm{H}, \mathrm{HCH}), 0.91\left(\mathrm{~s}, 3 \mathrm{H}, \mathrm{CH}_{3}\right), 0.82\left(\mathrm{~s}, 3 \mathrm{H}, \mathrm{CH}_{3}\right), 0.69\left(\mathrm{~s}, 3 \mathrm{H}, \mathrm{CH}_{3}\right) ;{ }^{13} \mathrm{C} \mathrm{NMR}$ $\left(\mathrm{CDCl}_{3}\right) \delta 215.8,142.2,132.3,129.7,128.2,124.8,124.0,87.5,52.0,50.9,48.5,44.8,43.7,41.6$, $38.2,36.8,30.0,26.1,20.9,20.5,18.8,18.7,11.1$.

\section{Detachment of the Auxiliary and Stereochemical Assignments}

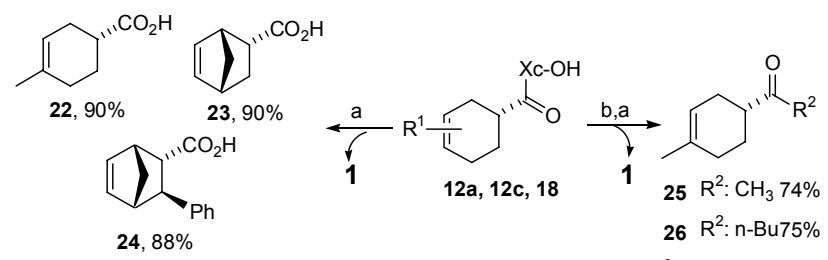

Conditions: (a). $\left(\mathrm{NH}_{4}\right)_{2} \mathrm{Ce}\left(\mathrm{NO}_{3}\right)_{6}(\mathrm{CAN})$ (3 equiv.), $\mathrm{CH}_{3} \mathrm{CN}-\mathrm{H}_{2} \mathrm{O}, 0^{\circ} \mathrm{C}, 10$ min. (b) $\mathrm{R}^{2} \mathrm{Li}$ (3 equiv.), THF, $0^{\circ} \mathrm{C}, 1.5 \mathrm{~h}$. 


\section{General procedure for the oxidation of Diels-Alder adducts}

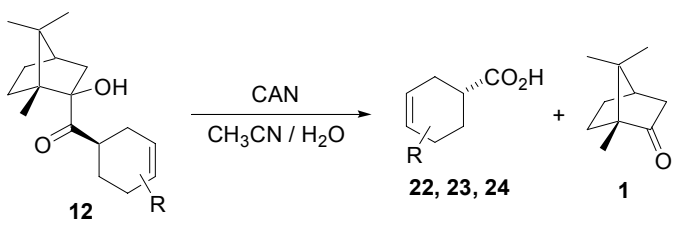

To a solution of the corresponding $\alpha$.-hydroxy ketone $\mathbf{1 2}$ $(1 \mathrm{mmol})$ in acetonitrile $(12 \mathrm{~mL})$ at $0{ }^{\circ} \mathrm{C}$ was added dropwise a solution of cerium ammonium nitrate (CAN) $(1.64 \mathrm{~g}, 3 \mathrm{mmol})$ in water $(6 \mathrm{~mL})$ and the mixture was stirred at the same temperature for 10 minutes. Then water $(3 \mathrm{~mL})$ was added and the mixture was extracted with $\mathrm{CH}_{2} \mathrm{Cl}_{2}(2 \times 20 \mathrm{~mL})$. The combined organic extracts were washed with a saturated aqueous solution of $\mathrm{NaHCO}_{3}(2 \times 10 \mathrm{~mL})$, dried over $\mathrm{MgSO}_{4}$, filtered, and the solvent evaporated to afford the starting (R)-(+)-camphor in $90 \%$ yield. The basic aqueous layer was acidified by adding concentrate $\mathrm{HCl}$ and then extracted with $\mathrm{CH}_{2} \mathrm{Cl}_{2}(2$ x $20 \mathrm{~mL}$ ). The combined extracts were dried over $\mathrm{MgSO}_{4}$, filtered and the solvent removed under reduced pressure. The crude product was purified by column chromatography (eluant: ethyl acetate/hexane 1:30) to afford the corresponding carboxylic acid.

\section{(1R)-4-methylcyclohex-3-enecarboxylic $\operatorname{acid}^{3} . \underline{22}$}

The title compound was prepared according to general procedure from 12a $(0.276 \mathrm{~g}, 1 \mathrm{mmol}) .0 .126$ g (90\%); m.p. $80{ }^{\circ} \mathrm{C}$; $[\alpha]_{\mathrm{D}}^{25}+71.2\left(c\right.$ 2.22, $\mathrm{Cl}_{2} \mathrm{CH}_{2}$ ); IR (neat, $\mathrm{cm}^{-1}$ ) $2986(\mathrm{OH}), 1706(\mathrm{CO}) ;{ }^{1} \mathrm{H} \mathrm{NMR}$ $\left(\mathrm{CDCl}_{3}\right) \delta 11.78(\mathrm{sb}, 1 \mathrm{H}, \mathrm{OH}), 5.35(\mathrm{sb}, 1 \mathrm{H}, \mathrm{HC}=\mathrm{C}), 2.58-2.44(\mathrm{~m}, 1 \mathrm{H}, \mathrm{HCCO}), 2.31-2.16(\mathrm{~m}, 2 \mathrm{H}$, $\mathrm{HCH}), 2.07-1.95(\mathrm{~m}, 3 \mathrm{H}, \mathrm{HCH}), 1.80-1.58(\mathrm{~m}, 1 \mathrm{H}, \mathrm{HCH}), 1.63\left(\mathrm{~s}, 3 \mathrm{H}, \mathrm{CH}_{3}\right) ;{ }^{13} \mathrm{C} \mathrm{NMR}\left(\mathrm{CDCl}_{3}\right) \delta$ 182.7, 133.7, 118.9, 39.1, 29.1, 27.4, 25.2, 23.5; Anal. calcd for $\mathrm{C}_{8} \mathrm{H}_{12} \mathrm{O}_{2}$ (140.2) C, 68.53; H, 8.64. Found: C, 68.76; H, 8.53.

\section{(1R, 2R, 4R)-bicyclo[2.2.1]hept-5-ene-2-carboxylic acid ${ }^{4} . \underline{23}$}

The title compound was prepared according to general procedure from 12c $(0.274 \mathrm{~g}, 1 \mathrm{mmol})$. 0.124 g (90\%); m.p. 34-35 ${ }^{\circ} \mathrm{C}$; $[\alpha]_{\mathrm{D}}^{25}+140.0$ (c 1.0, EtOH); IR (neat, $\mathrm{cm}^{-1}$ ) 2986 (OH), 1706 (CO); ${ }^{1} \mathrm{H}$ NMR $\left(\mathrm{CDCl}_{3}\right) \delta 6.19(\mathrm{dd}, 1 \mathrm{H}, \mathrm{HC}=\mathrm{C}, \mathrm{J}=2.9 \mathrm{~Hz}, \mathrm{~J}=5.7 \mathrm{~Hz}), 5.97(\mathrm{dd}, 1 \mathrm{H}, \mathrm{HC}=\mathrm{C}, \mathrm{J}=2.9 \mathrm{~Hz}$, $\left.\mathrm{J}^{\prime}=5.5 \mathrm{~Hz}\right), 3.21(\mathrm{~m}, 1 \mathrm{H}, \mathrm{HCC}), 3.02-2.89(\mathrm{~m}, 1 \mathrm{H}, \mathrm{HCCO}), 2.90(\mathrm{~m}, 1 \mathrm{H}, \mathrm{HCC}), 1.96-1.83(\mathrm{~m}, 1 \mathrm{H}$, $\mathrm{HCH}), 1.47-1.24(\mathrm{~m}, 3 \mathrm{H}, \mathrm{HCH}) ;{ }^{13} \mathrm{C} \mathrm{NMR}\left(\mathrm{CDCl}_{3}\right) \delta 181.0,137.8,132.3,49.7,45.7,43.3,42.6$, 29.1; Anal. calcd for $\mathrm{C}_{8} \mathrm{H}_{10} \mathrm{O}_{2}$ (138.18) C, 69.53; H, 7.31. Found: C, 69.49; H, 7.25.

\section{(1R, 2S, 3S, 4S)-3-phenylbicyclo[2.2.1]hept-5-ene-2-carboxylic acid. $\underline{24}$}

The title compound was prepared according to general procedure from $\mathbf{1 8}(0.350 \mathrm{~g}, 1 \mathrm{mmol}) .0 .188$ g (88\%); m.p. $41{ }^{\circ} \mathrm{C} ;[\alpha]_{\mathrm{D}}^{25}+126.0\left(c\right.$ 1.0, $\left.\mathrm{Cl}_{2} \mathrm{CH}_{2}\right)$; IR (neat, $\left.\mathrm{cm}^{-1}\right) 2975(\mathrm{OH}), 1700(\mathrm{CO}) ;{ }^{1} \mathrm{H}$ NMR $\left(\mathrm{CDCl}_{3}\right) \delta 7.35-7.17(\mathrm{~m}, 5 \mathrm{H}$, arom.), $6.43(\mathrm{dd}, 1 \mathrm{H}, \mathrm{HC}=\mathrm{C}, \mathrm{J}=3.3 \mathrm{~Hz}, \mathrm{~J}=5.9 \mathrm{~Hz}), 6.18(\mathrm{dd}, 1 \mathrm{H}$, $\left.\mathrm{HC}=\mathrm{C}, \mathrm{J}=2.6 \mathrm{~Hz}, \mathrm{~J}^{\prime}=5.5 \mathrm{~Hz}\right) 3.32(\mathrm{~m}, 1 \mathrm{H}, \mathrm{HCC}), 3.10-3.04(\mathrm{~m}, 3 \mathrm{H}, \mathrm{HCCO}, \mathrm{HCC}, \mathrm{HCPh}), 1.79(\mathrm{~d}$, $1 \mathrm{H}, \mathrm{HCC}, \mathrm{J}=9.3 \mathrm{~Hz}), 1.60(\mathrm{dd}, 1 \mathrm{H}, \mathrm{HCH}, \mathrm{J}=1.8 \mathrm{~Hz}, \mathrm{~J}=8.8 \mathrm{~Hz}) ;{ }^{13} \mathrm{C} \mathrm{NMR}\left(\mathrm{CDCl}_{3}\right) \delta 181.1,143.8$, 139.1, 134.5, 128.4, 127.3, 126.0, 52.5, 48.2, 47.3, 47.2, 46.2; Anal. calcd for $\mathrm{C}_{14} \mathrm{H}_{14} \mathrm{O}_{2}(381.58) \mathrm{C}$,

\footnotetext{
${ }^{3}[\alpha]_{\mathrm{D}}^{20}=+69.2\left(\mathrm{c}=0.48, \mathrm{CHCl}_{3}\right)$ Burke M. J.; Allan M. M.; Parvez M.; Keay B. A. Tetrahedron Asymmetry 2000, 2733-2739.

${ }^{4}$ Compound (-)-23: $[\alpha]_{\mathrm{D}}{ }^{20}=-139.0(\mathrm{c}=1.38, \mathrm{EtOH})$ Choy W.; Reed L.A.; Masamune S. J. Org. Chem. 1983, 48, 1137-1139.
} 
78.47; H, 6.60. Found: $\mathrm{C}, 78.59 ; \mathrm{H}, 6.65$. Optical rotation for the corresponding benzyl ester: $[\alpha]_{\mathrm{D}}^{25}+119.2\left(c 1.2, \mathrm{CH}_{2} \mathrm{Cl}_{2}\right)^{5}$.

\section{General procedure for ketone synthesis}

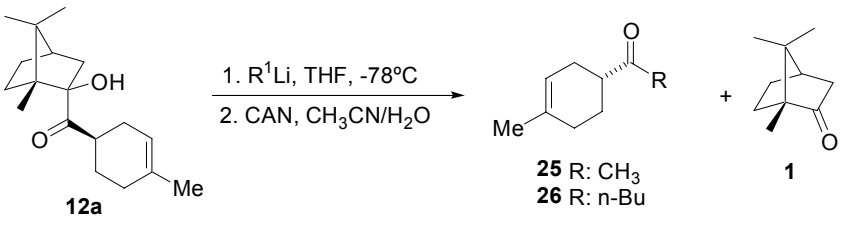

nBuLi (2.5 $\mathrm{M}$ in hexanes, $1.2 \mathrm{~mL}, 3 \mathrm{mmol})$ or $\mathrm{MeLi}\left(1.4 \mathrm{M}\right.$ in $\left.\mathrm{Et}_{2} \mathrm{O}, 2.14 \mathrm{~mL}, 3 \mathrm{mmol}\right)$ was added to a solution of the corresponding $\alpha$ 'hydroxy ketone $12 \mathrm{a}(0.276 \mathrm{~g}, 1 \mathrm{mmol})$ in THF $(3 \mathrm{~mL})$ at $-78^{\circ} \mathrm{C}$ and the solution was stirred at $0{ }^{\circ} \mathrm{C}$ for $1.5 \mathrm{~h}$. The reaction mixture was quenched with a saturated aqueous solution of $\mathrm{NH}_{4} \mathrm{Cl}(5$ $\mathrm{ml}$ ) and the resulting mixture was allowed to warm to room temperature, after which the layers were separated and the aqueous layer was extracted twice with $\mathrm{CH}_{2} \mathrm{Cl}_{2}(15 \mathrm{~mL})$. The combined organic layers were dried over $\mathrm{MgSO}_{4}$ and the solvent removed under reduced pressure. The residue thus obtained was subjected to oxidative scission by treatment with CAN, under the same conditions reported above. The resulting crude product 25 or 26 was purified by column chromatography (eluant: ethyl acetate/hexane 1:30).

\section{Methyl-[(1R)-4-methylcyclohex-3-enyl]ketone. $\underline{25}$}

The title compound was prepared according to general procedure from 12a and MeLi. $0.102 \mathrm{~g}$ (74\%); b.p. $90{ }^{\circ} \mathrm{C} / 15 \mathrm{mmHg} ;[\alpha]_{\mathrm{D}}^{25}+26.0$ (c 0.36, $\mathrm{Cl}_{2} \mathrm{CH}_{2}$ ); IR (neat, $\mathrm{cm}^{-1}$ ) 1711 (CO); ${ }^{1} \mathrm{H} \mathrm{NMR}$ $\left(\mathrm{CDCl}_{3}\right) \delta 5.37(\mathrm{~m}, 1 \mathrm{H}, \mathrm{HC}=\mathrm{C}), 2.53-2.43(\mathrm{~m}, 1 \mathrm{H}, \mathrm{HCC}), 2.19-1.83\left(\mathrm{~m}, 4 \mathrm{H}, \mathrm{CH}_{2}\right), 2.15(\mathrm{~s}, 3 \mathrm{H}$, $\left.\mathrm{CH}_{3} \mathrm{CO}\right), 1.67-1.48\left(\mathrm{~m}, 2 \mathrm{H}, \mathrm{CH}_{2}\right), 1.63\left(\mathrm{~s}, 3 \mathrm{H}, \mathrm{CH}_{3}\right) ;{ }^{13} \mathrm{C} \mathrm{NMR}\left(\mathrm{CDCl}_{3}\right) \delta 211.7,133.7,119.2,47.2$, $29.5,28.0,27.1,24.9,23.4$.

\section{Butyl-[(1R)-4-methylcyclohex-3-enyl]ketone. $\underline{26}$}

The title compound was prepared according to general procedure from 12a and nBuLi. $0.135 \mathrm{~g}$ (75\%); b.p. $95{ }^{\circ} \mathrm{C} / 18 \mathrm{mmHg} ;[\alpha]_{\mathrm{D}}^{25}+89.0$ (c 1.0, $\mathrm{Cl}_{2} \mathrm{CH}_{2}$ ); IR (neat, $\mathrm{cm}^{-1}$ ) 1706 (CO); ${ }^{1} \mathrm{H} \mathrm{NMR}$ $\left(\mathrm{CDCl}_{3}\right) \delta 5.37-5.36(\mathrm{t}, 1 \mathrm{H}, \mathrm{HC}=\mathrm{C}, \mathrm{J}=2.2 \mathrm{~Hz}), 2.58-2.35(\mathrm{~m}, 1 \mathrm{H}, \mathrm{HCCO}), 2.25-1.84(\mathrm{~m}, 6 \mathrm{H}, \mathrm{HCH})$, $1.62\left(\mathrm{~s}, 3 \mathrm{H}, \mathrm{CH}_{3}\right), 1.59-1.37(\mathrm{~m}, 4 \mathrm{H}, \mathrm{HCH}), 1.34-1.18(\mathrm{~m}, 2 \mathrm{H}, \mathrm{HCH}), 0.88\left(\mathrm{t}, 3 \mathrm{H}, \mathrm{CH}_{3}, \mathrm{~J}=7.3 \mathrm{~Hz}\right)$; ${ }^{13} \mathrm{C} \mathrm{NMR}\left(\mathrm{CDCl}_{3}\right) \delta 214.0,133.6,119.3,46.5,40.5,29.6,27.2,25.9,25.1,23.5,22.5,14.0$.

\section{Determination of isomeric ratios}

Diastereomeric and endo:exo ratios of the Diels-Alder adducts were determined by ${ }^{13} \mathrm{C}$ NMR and/or by HPLC, using a Merck LiChrospher 100 RP-18 column (eluent acetonitrile/water 70:30, flow rate $1 \mathrm{~mL} / \mathrm{min}$ ).

The enantiomeric purity of carboxylic acids $\mathbf{2 2}$ and $\mathbf{2 3}$ was determined by HPLC analysis of their methyl esters using a Chiralcel OD-H and OB-H columns, respectively, and hexane as the eluant (flow $0.5 \mathrm{~mL} / \mathrm{min}$ ), and it was confirmed by comparison of the chromatograms with those corresponding to racemic products. The enantiomeric purity of ketone $\mathbf{2 5}$ was determined by HPLC

${ }^{5}[\alpha]_{\mathrm{D}}^{20}=+121\left(\mathrm{c}=1.33, \mathrm{CHCl}_{3}\right)$ Evans D. A.; Chapman K. T.; Bisaha J. J. Am. Chem. Soc. 1988, 110, 12381256 . 
analysis, using a Chiralcel OB-H column and a mixture isopropanol/hexane 2:98 as the eluant (flow $0.5 \mathrm{~mL} / \mathrm{min}$ ), and it was confirmed by comparison of the chromatogram with that of the racemic ketone. The enantiomeric purity of ketone $\mathbf{2 6}$ was determined by HPLC analysis using a Chiralcel OD-R column and a mixture mathanol/water $0.32 / 0.18$ as the eluant (flow $0.5 \mathrm{~mL} / \mathrm{min}$ ) and it was confirmed by comparison of the chromatogram with that of the racemic ketone.

The mixture of regioisomers from the reaction of $\alpha$ '-hydroxy vinyl ketone 3 with isoprene was determined by ${ }^{13} \mathrm{C}$ NMR analysis of the crude product, and it was confirmed by ${ }^{13} \mathrm{C}$ NMR analysis of the isomeric carboxylic acids formed from the oxidative cleavage of the adducts with cerium ammonium nitrate (CAN). 

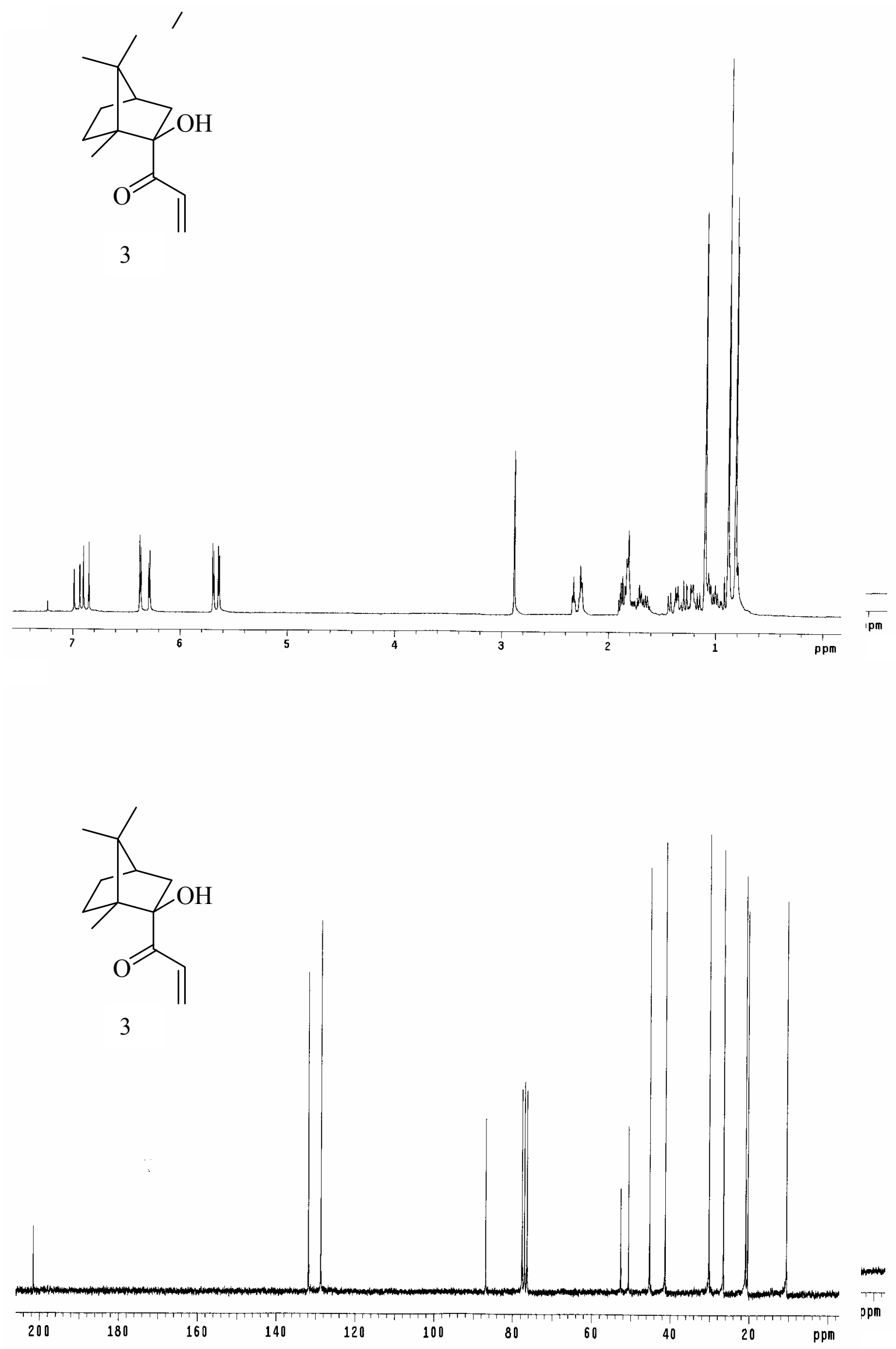

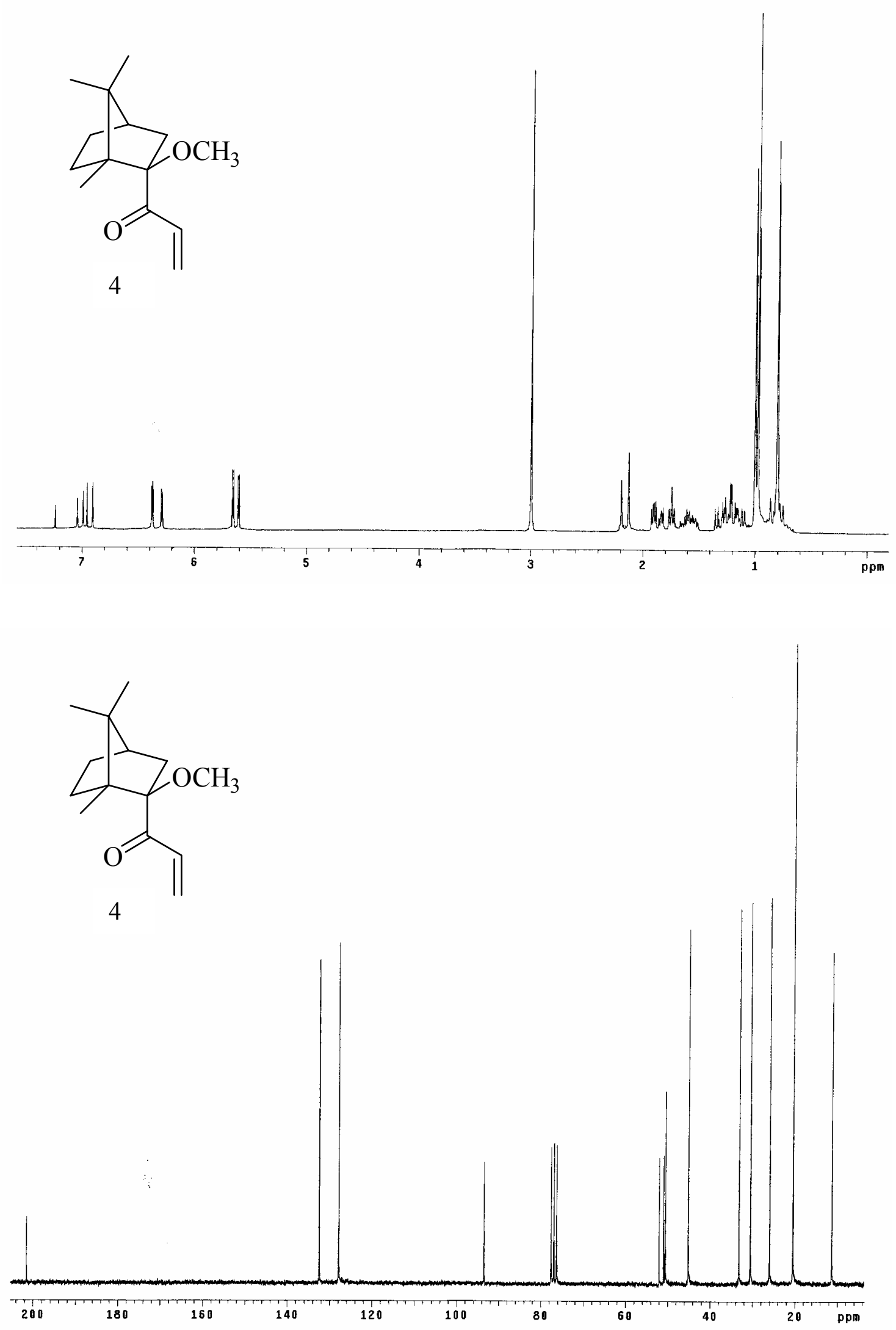

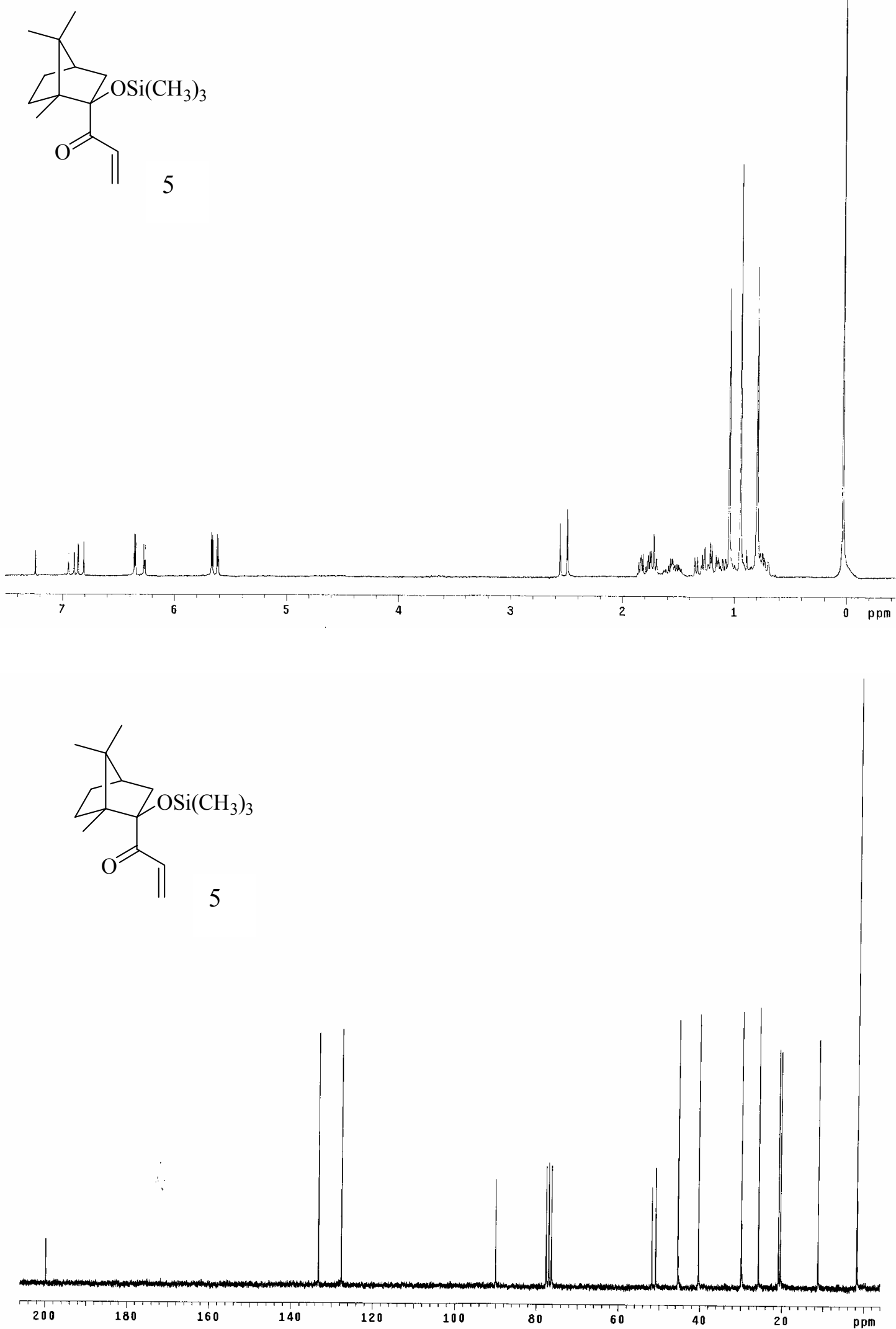


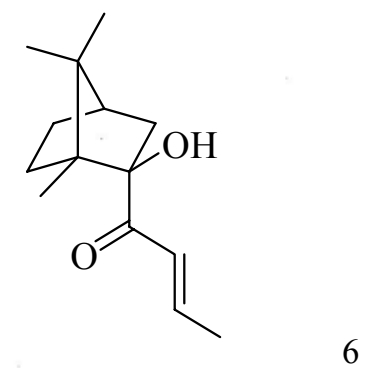

6
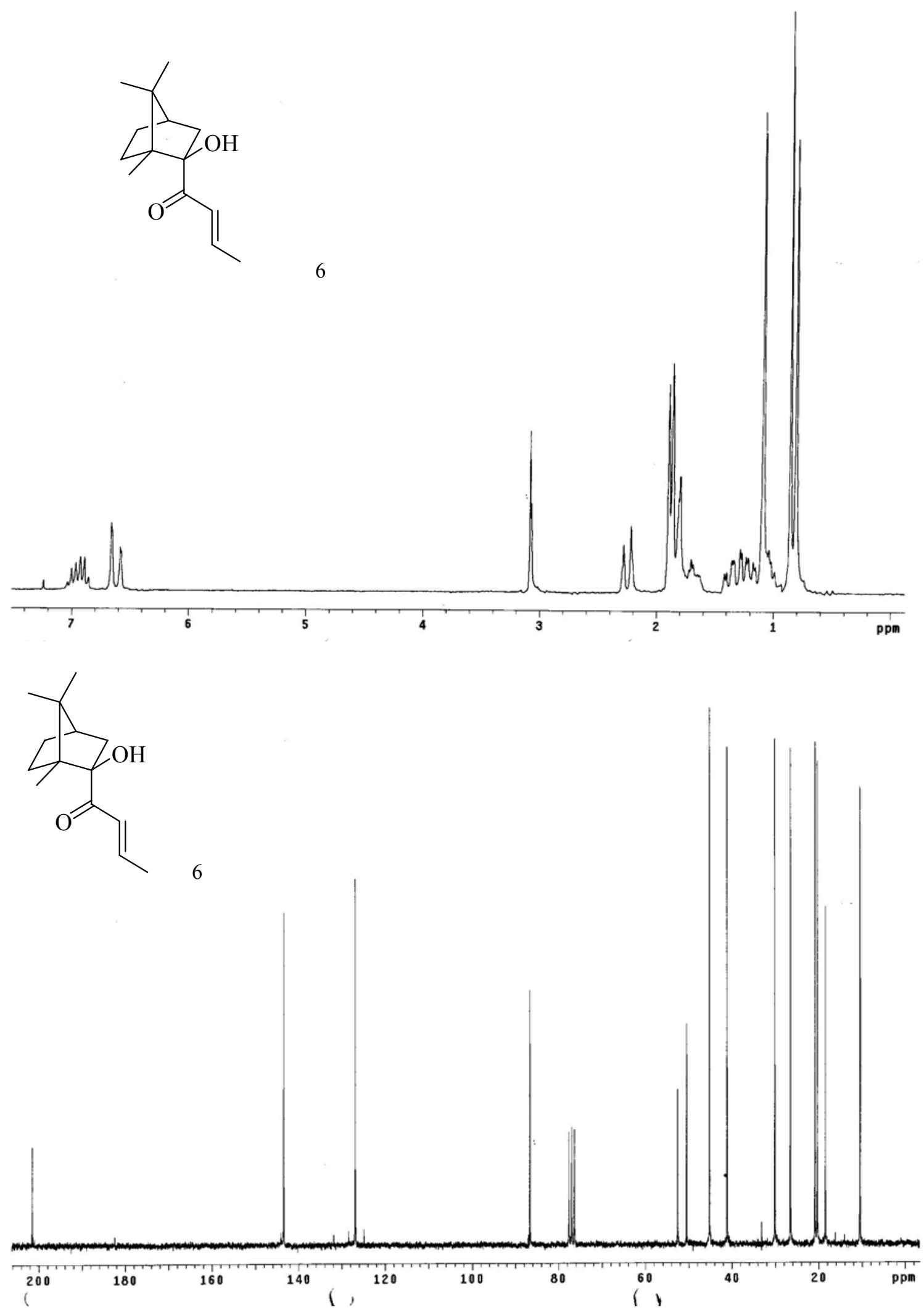

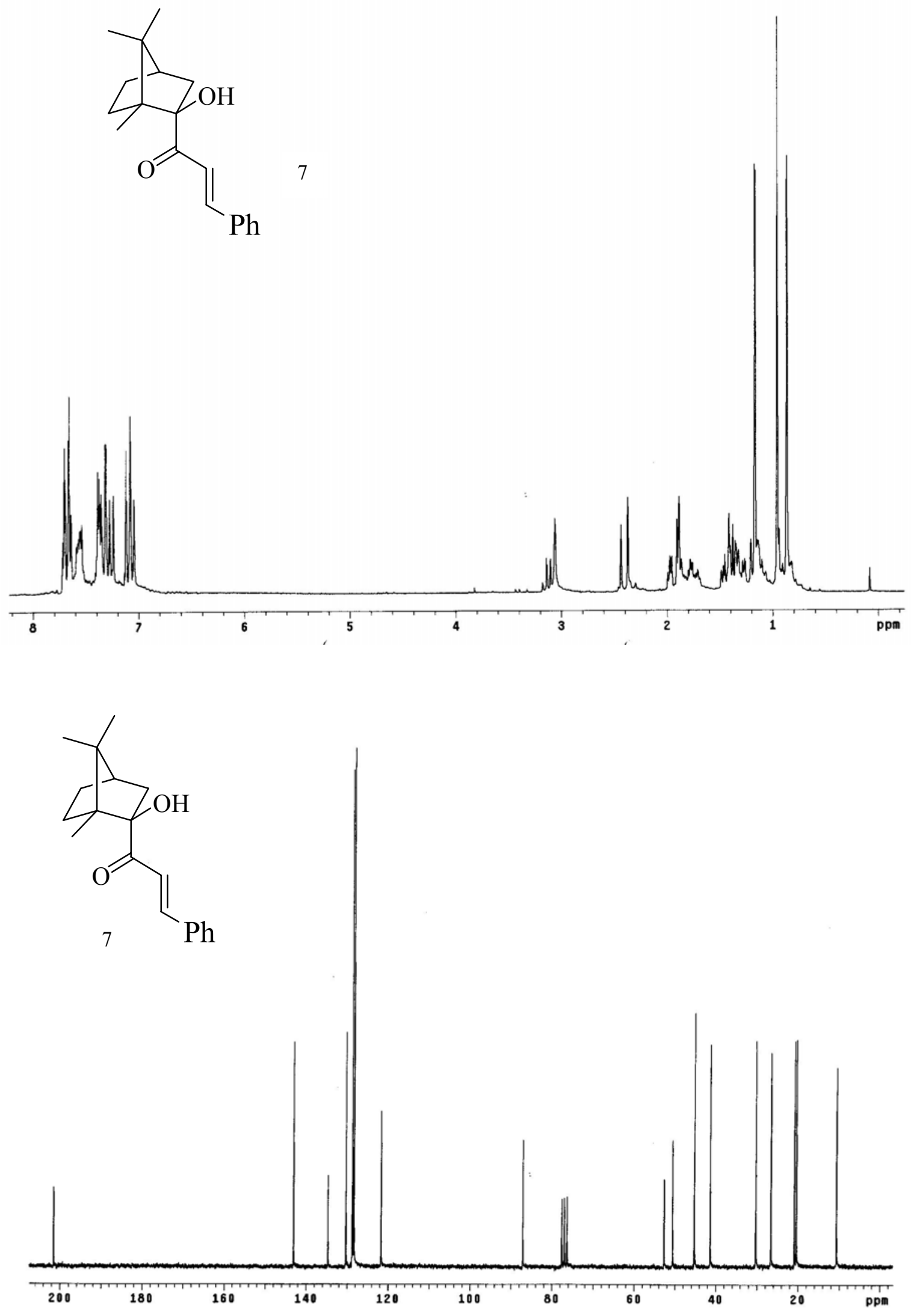

S16 

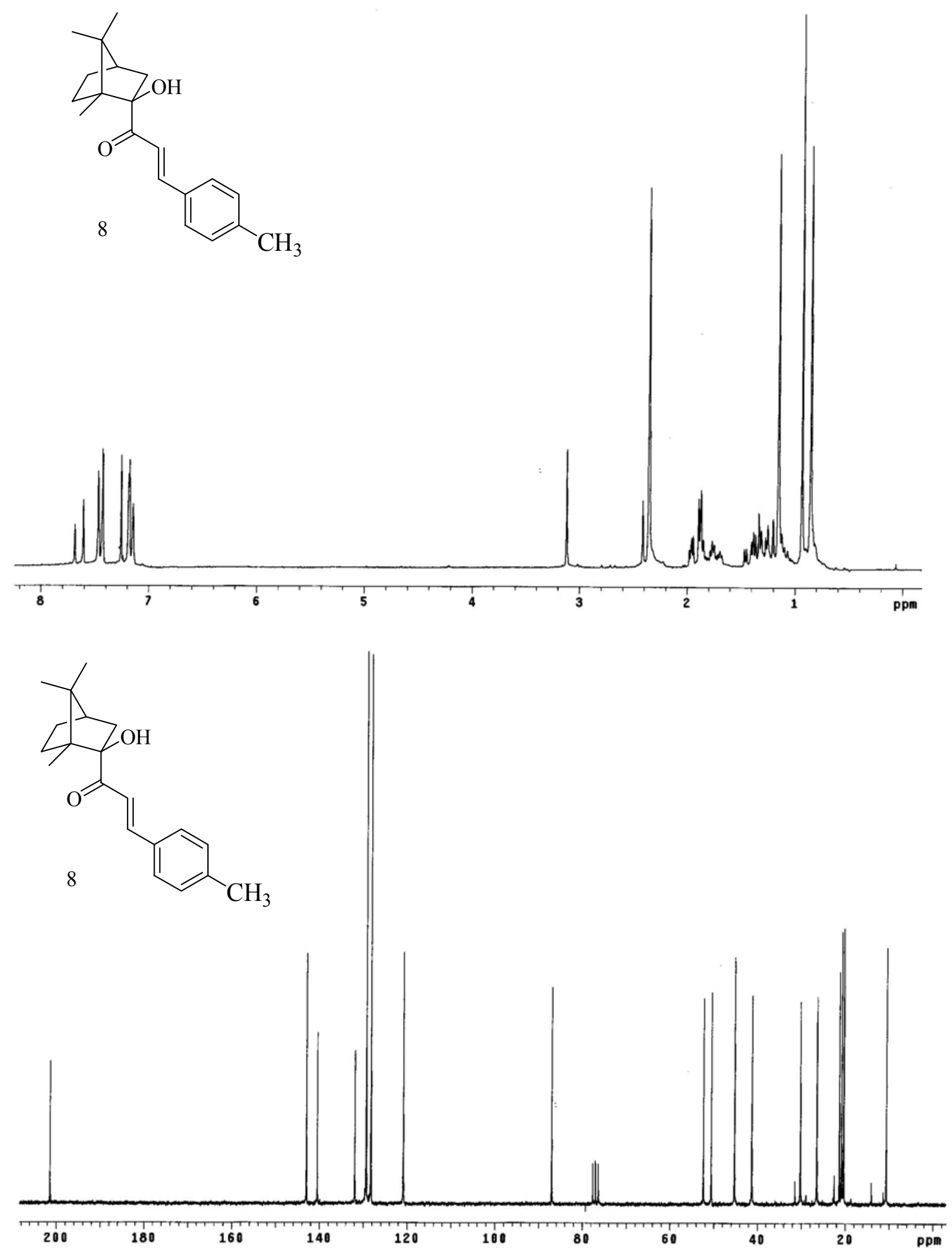

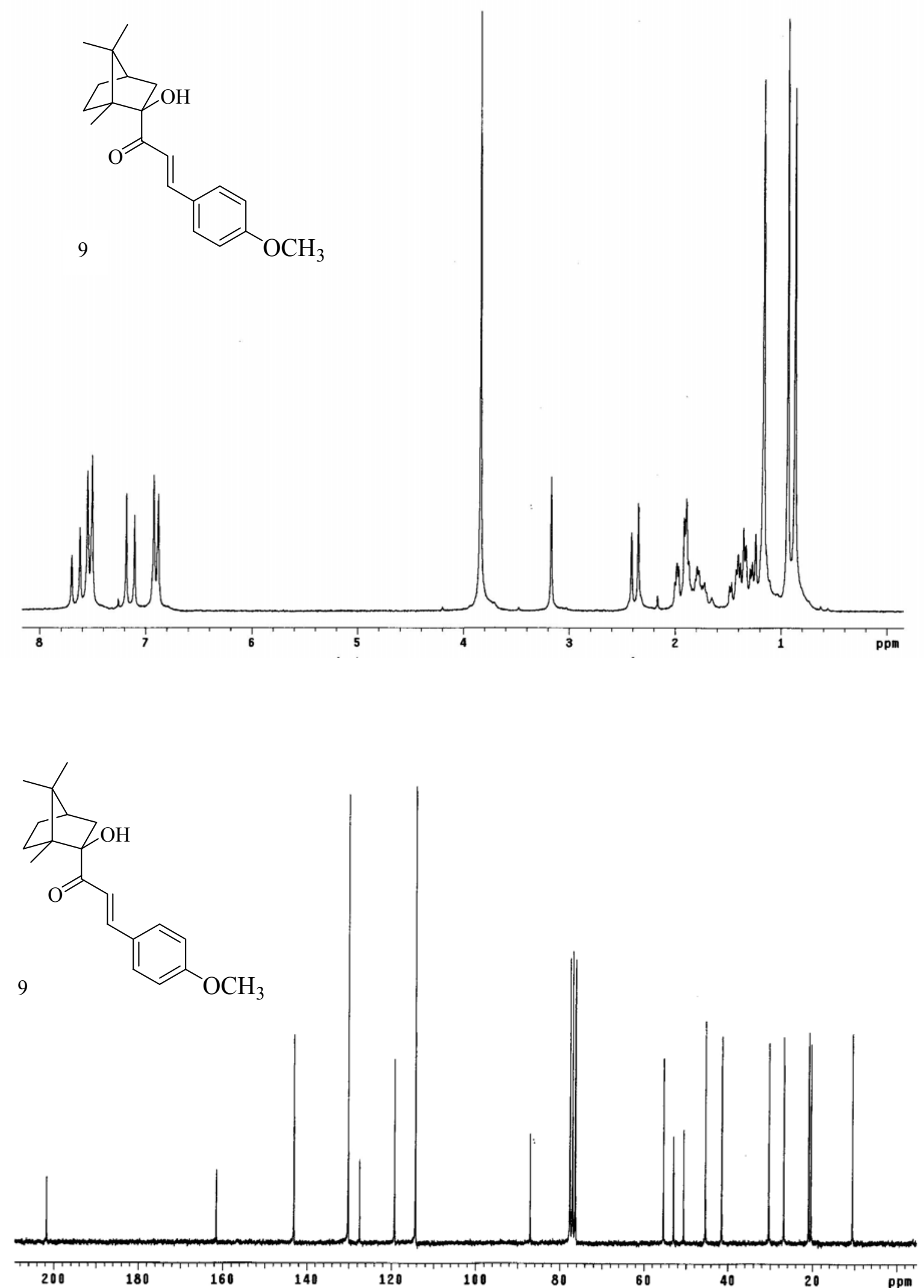

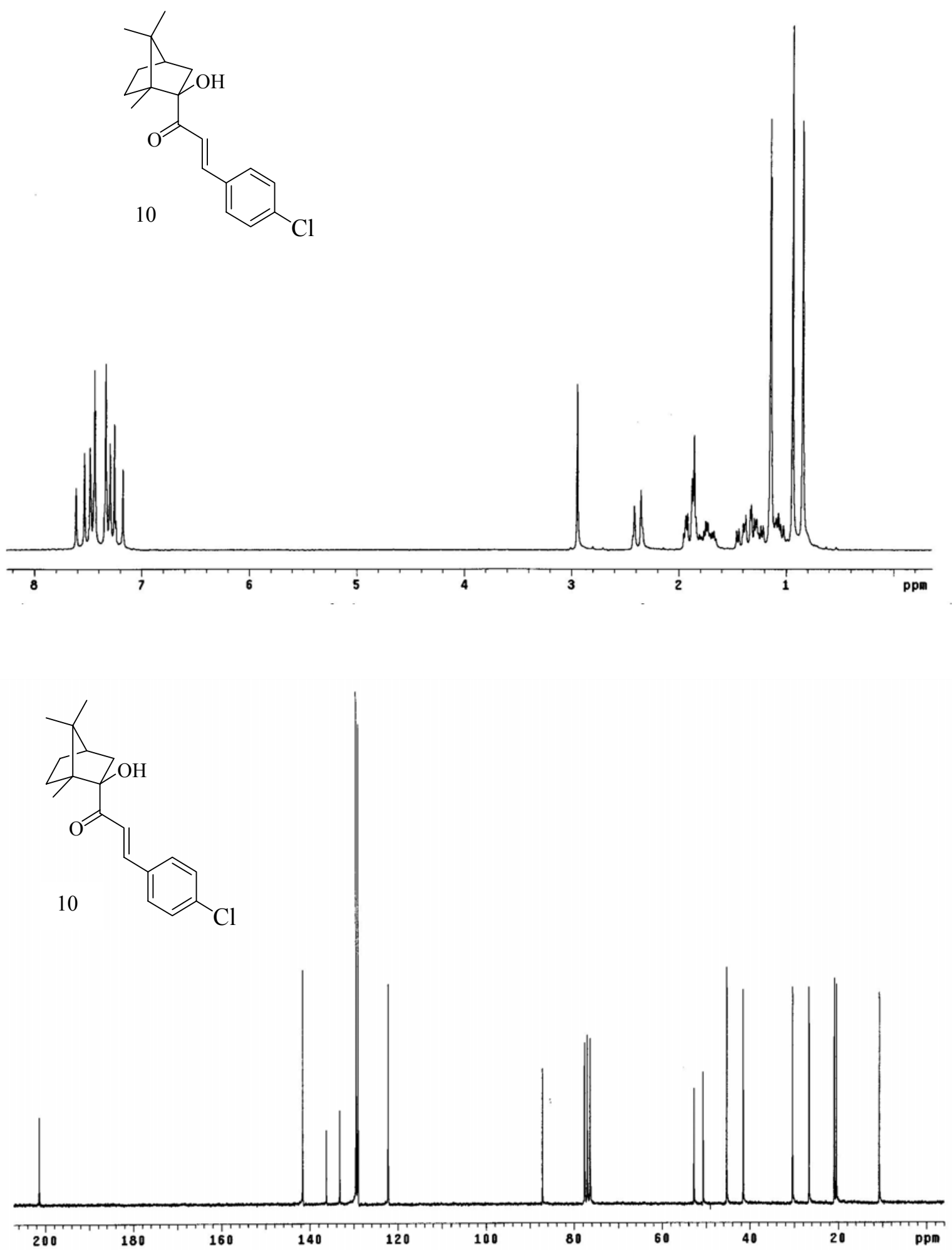


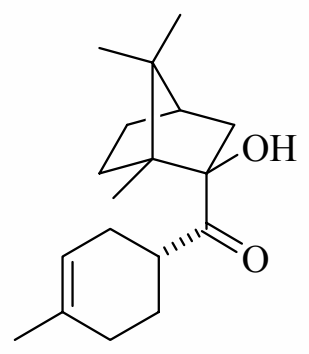

$12 \mathrm{a}$
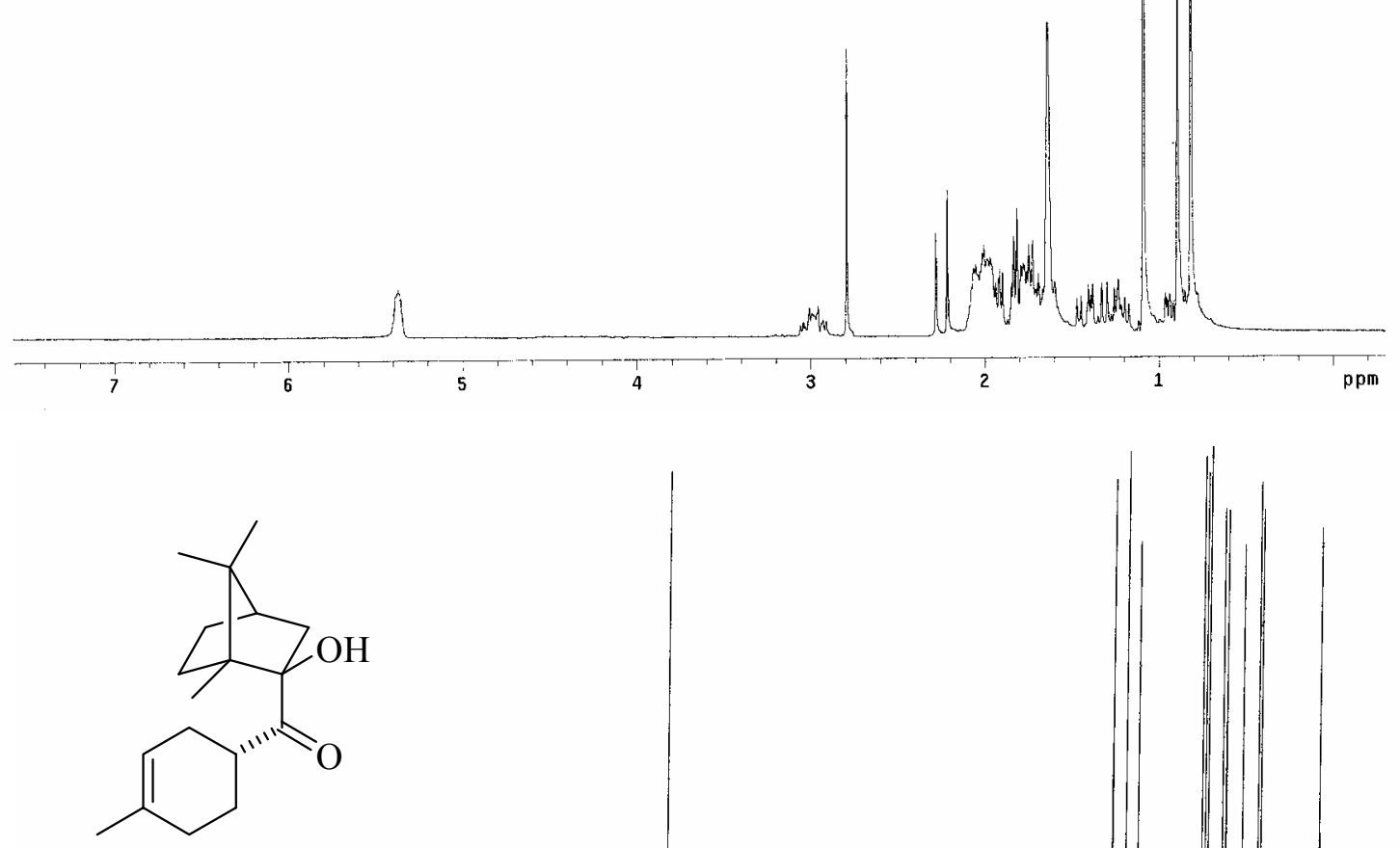

$12 \mathrm{a}$

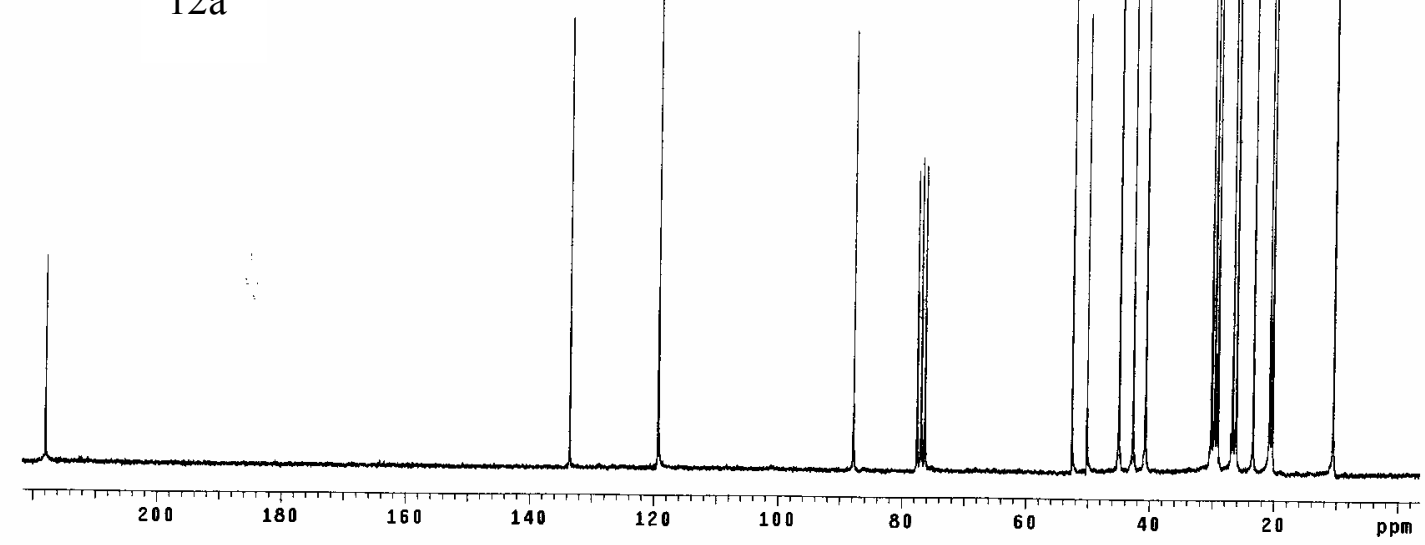




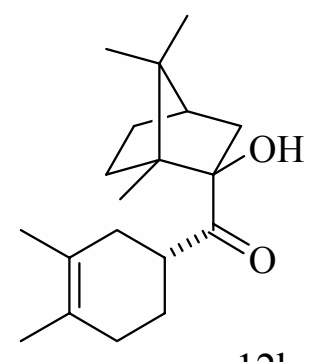

$12 b$

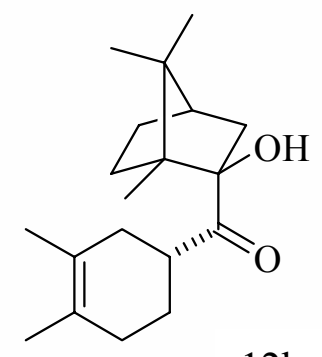

$12 b$

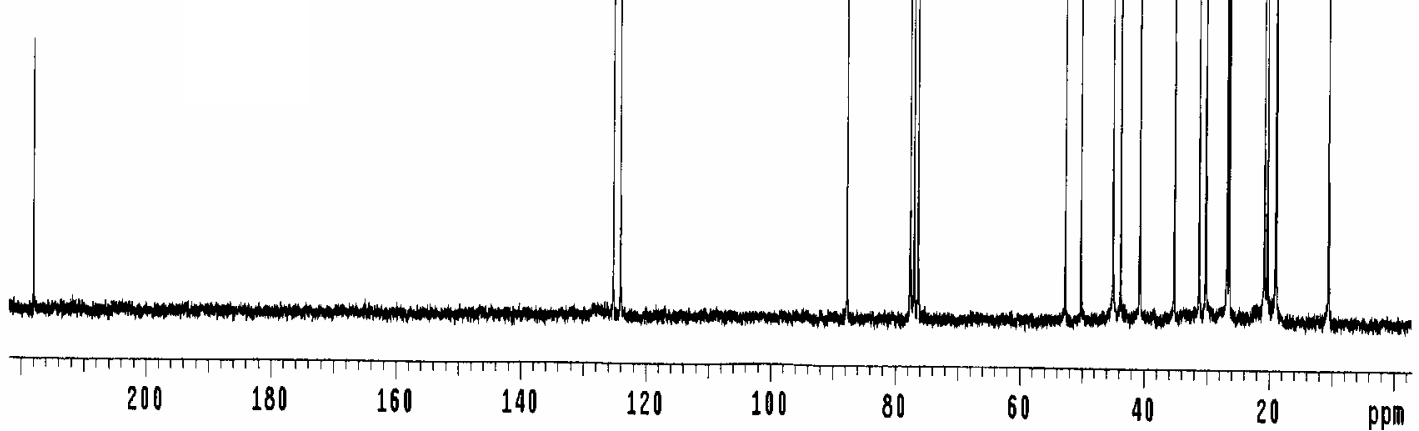




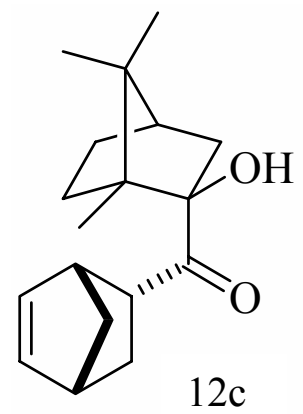

$12 \mathrm{c}$

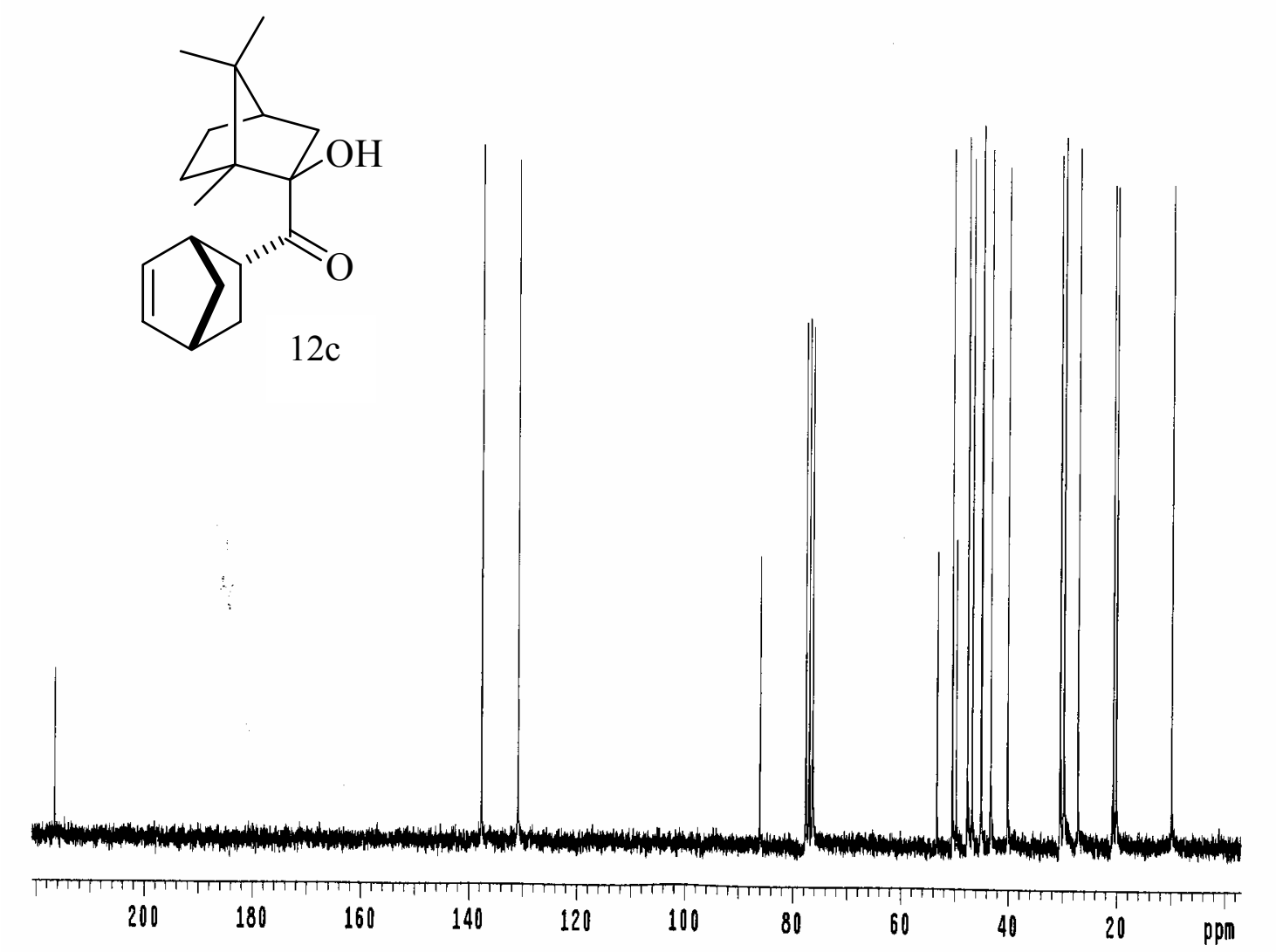




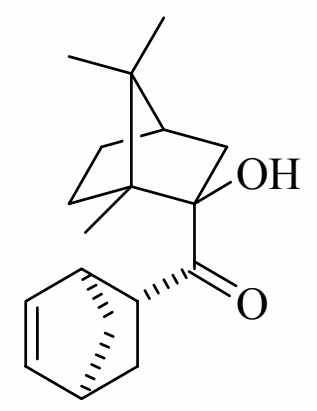

Exo

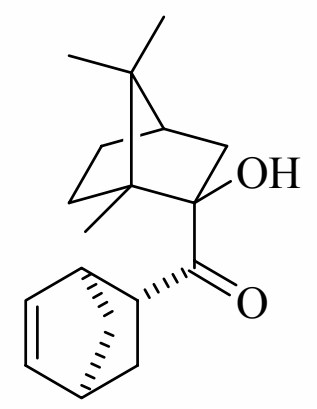

Exo
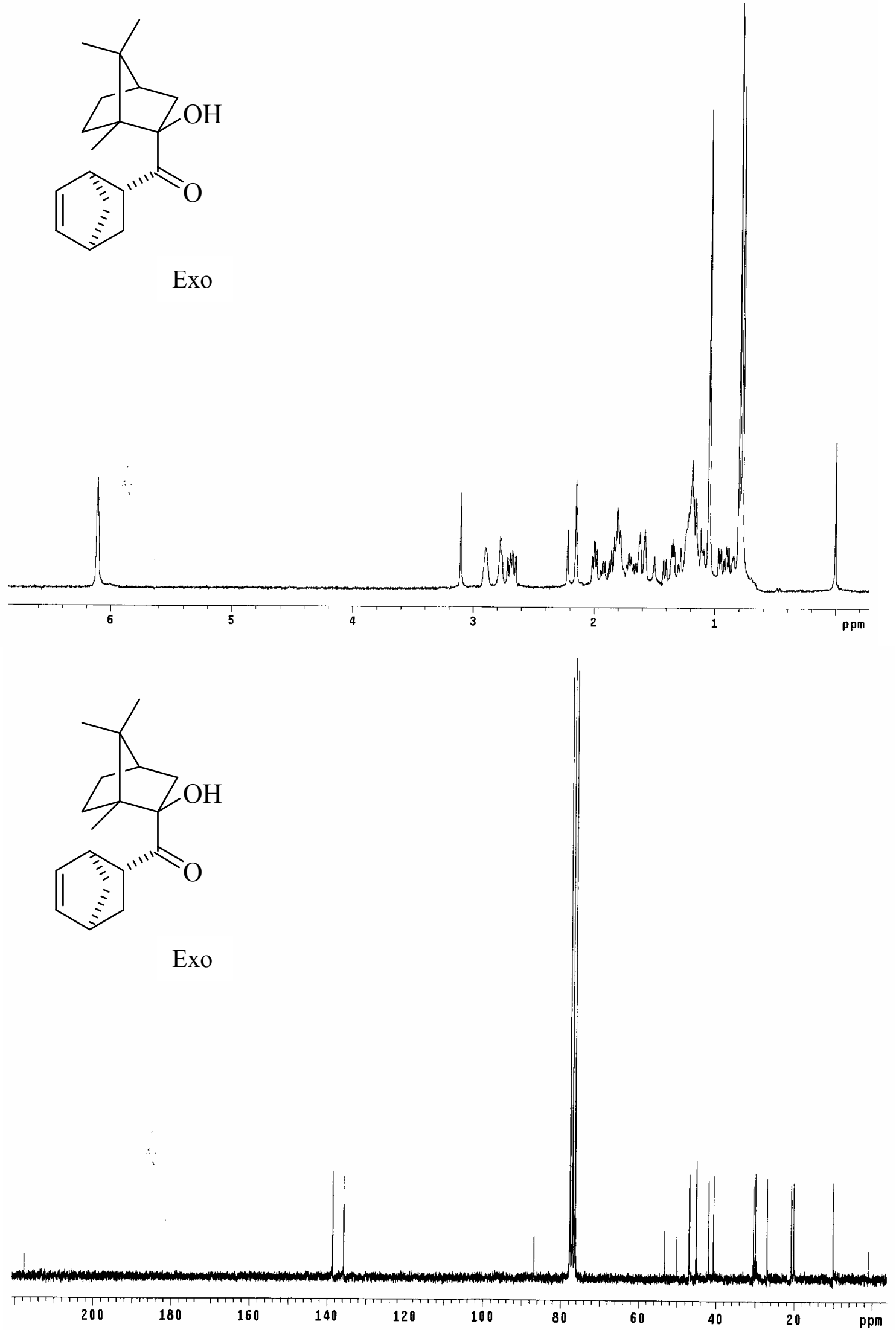

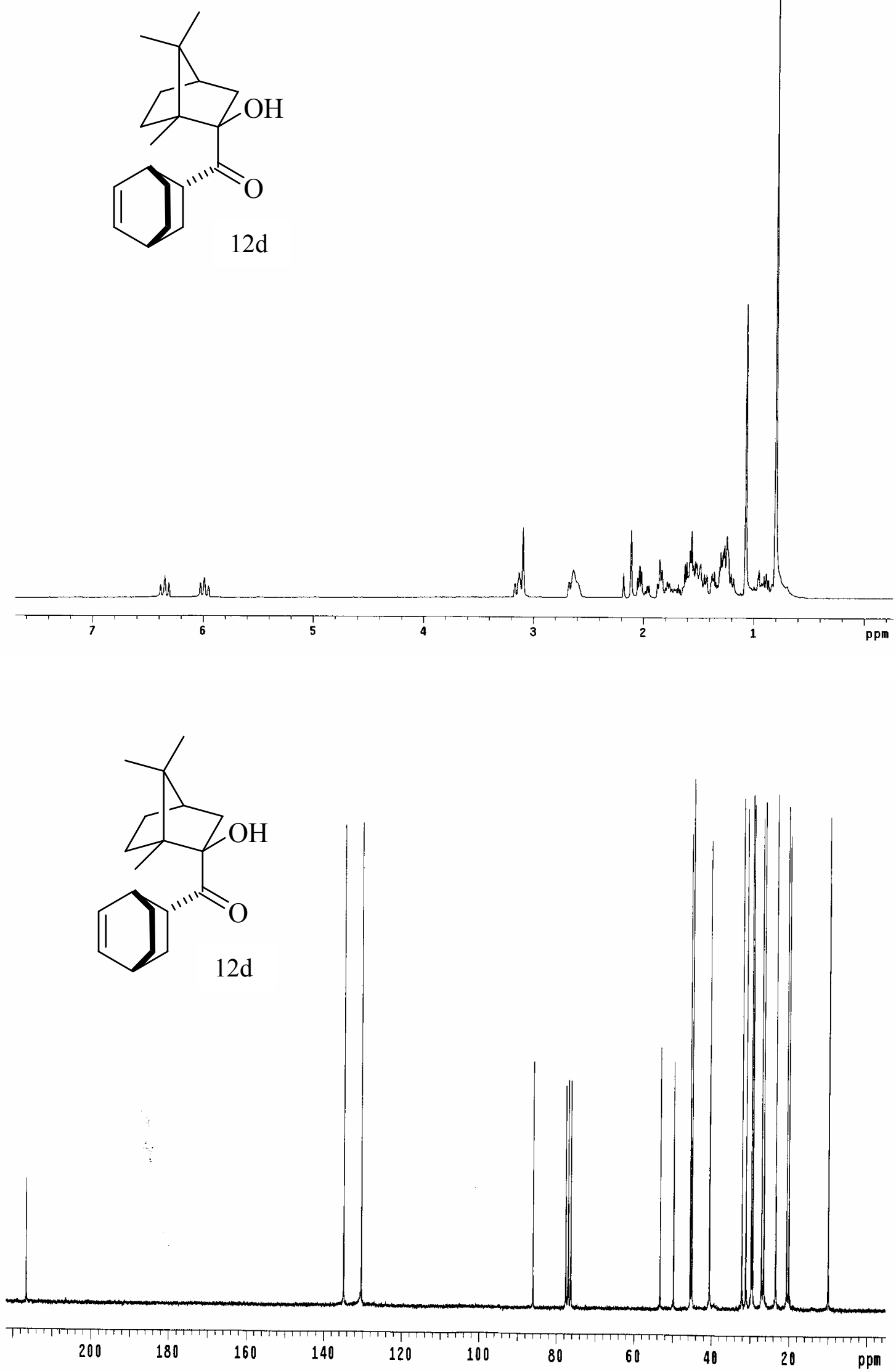


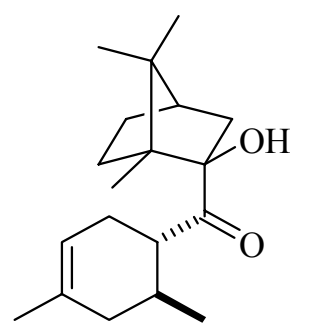

13

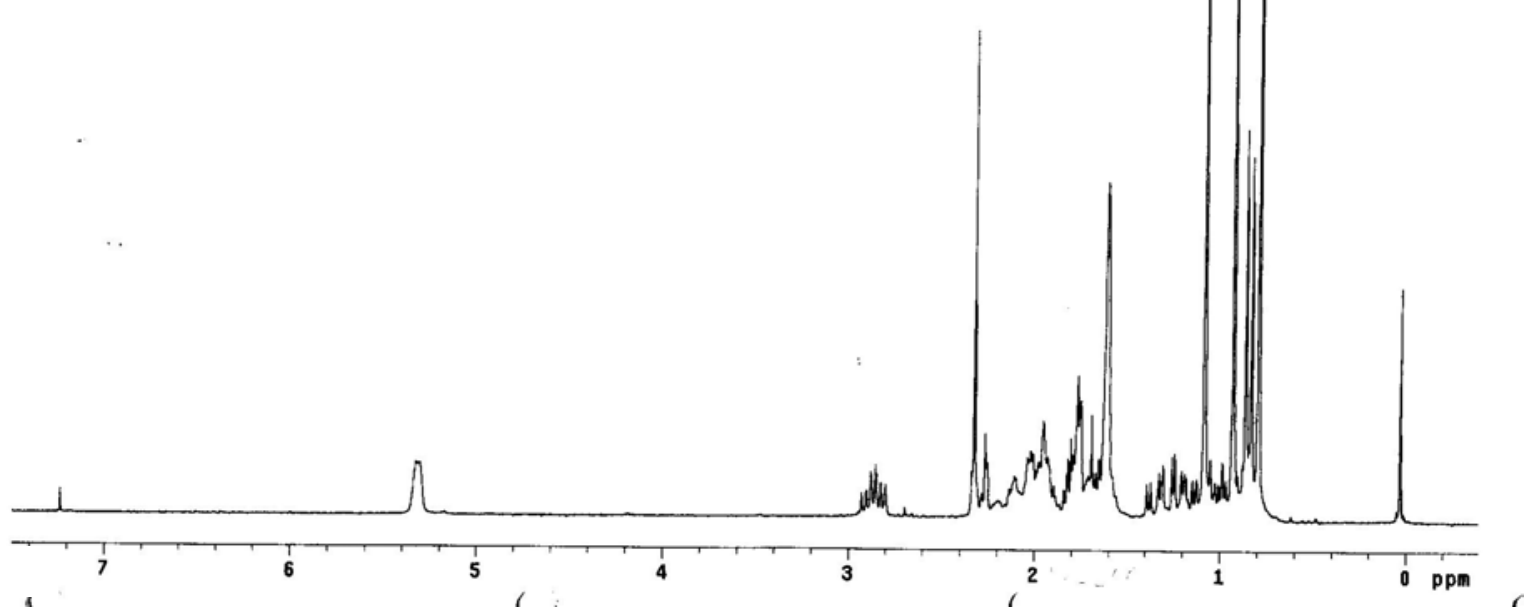

13

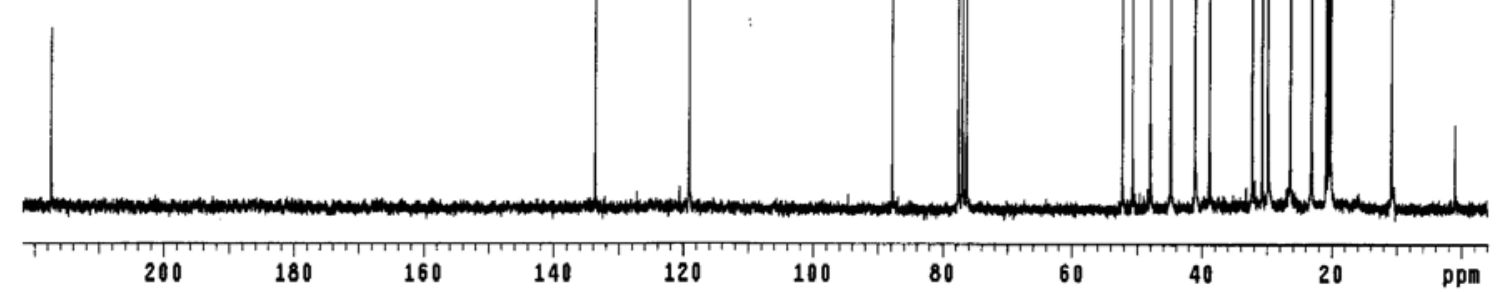



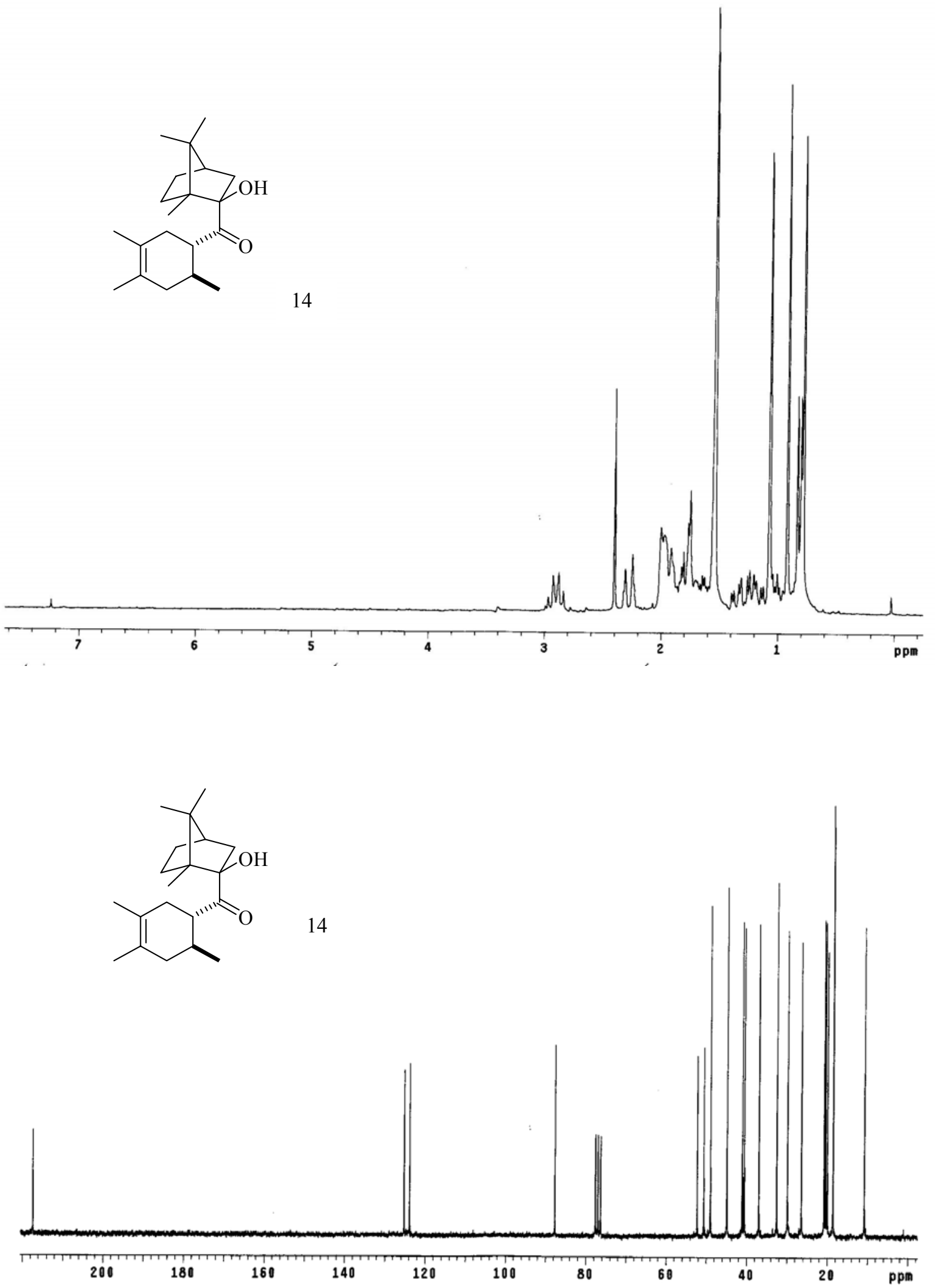


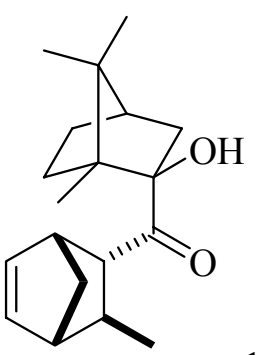

15
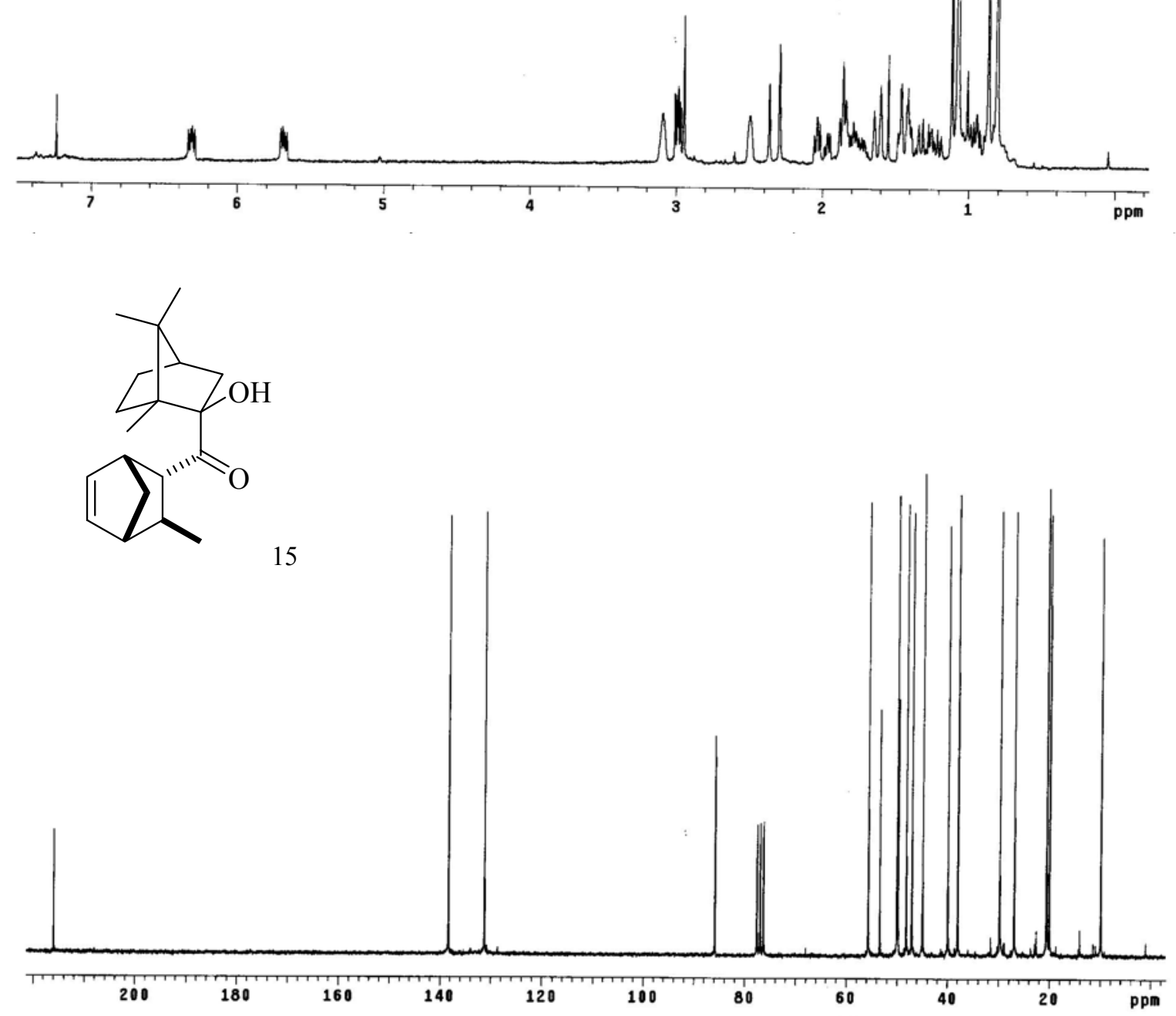

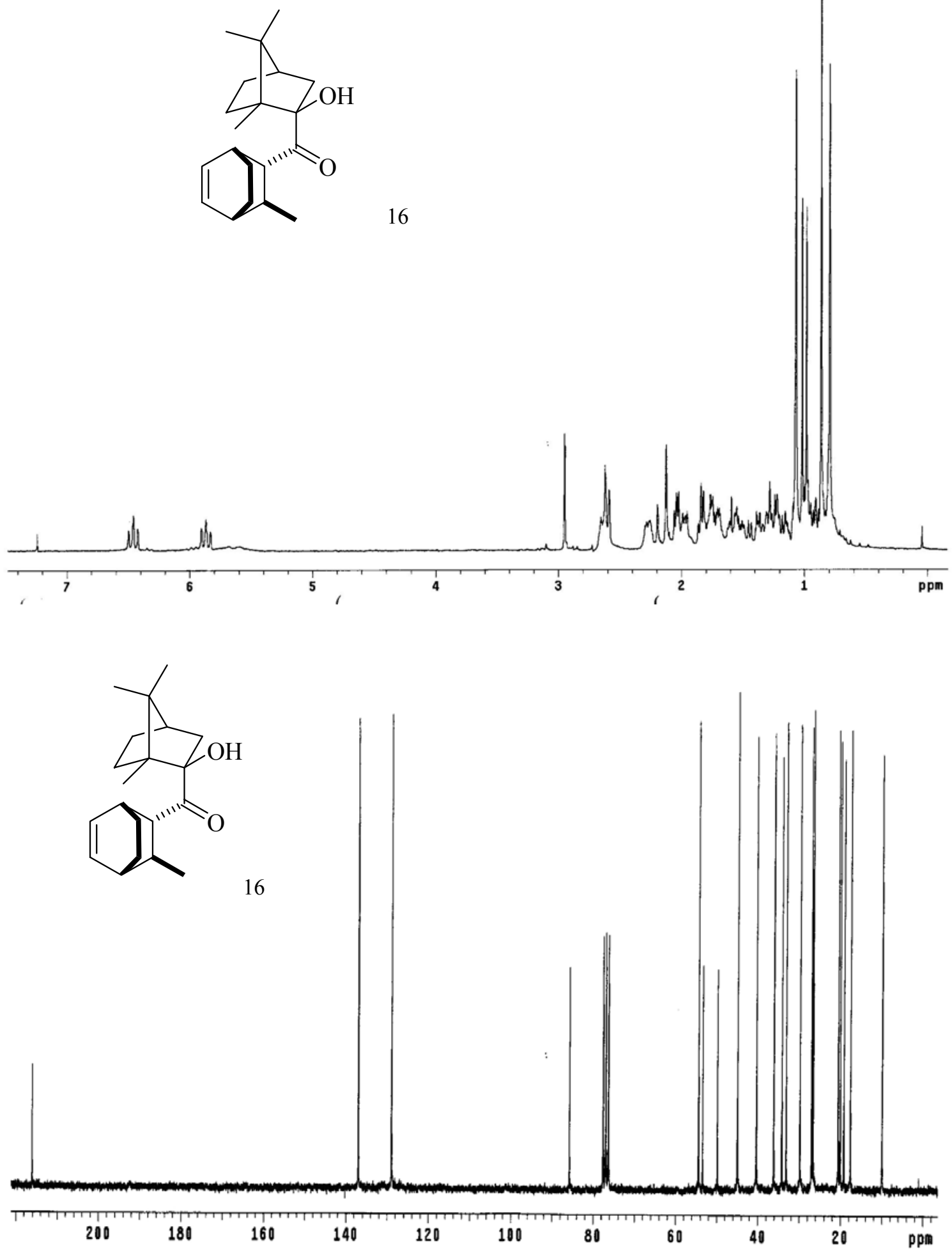

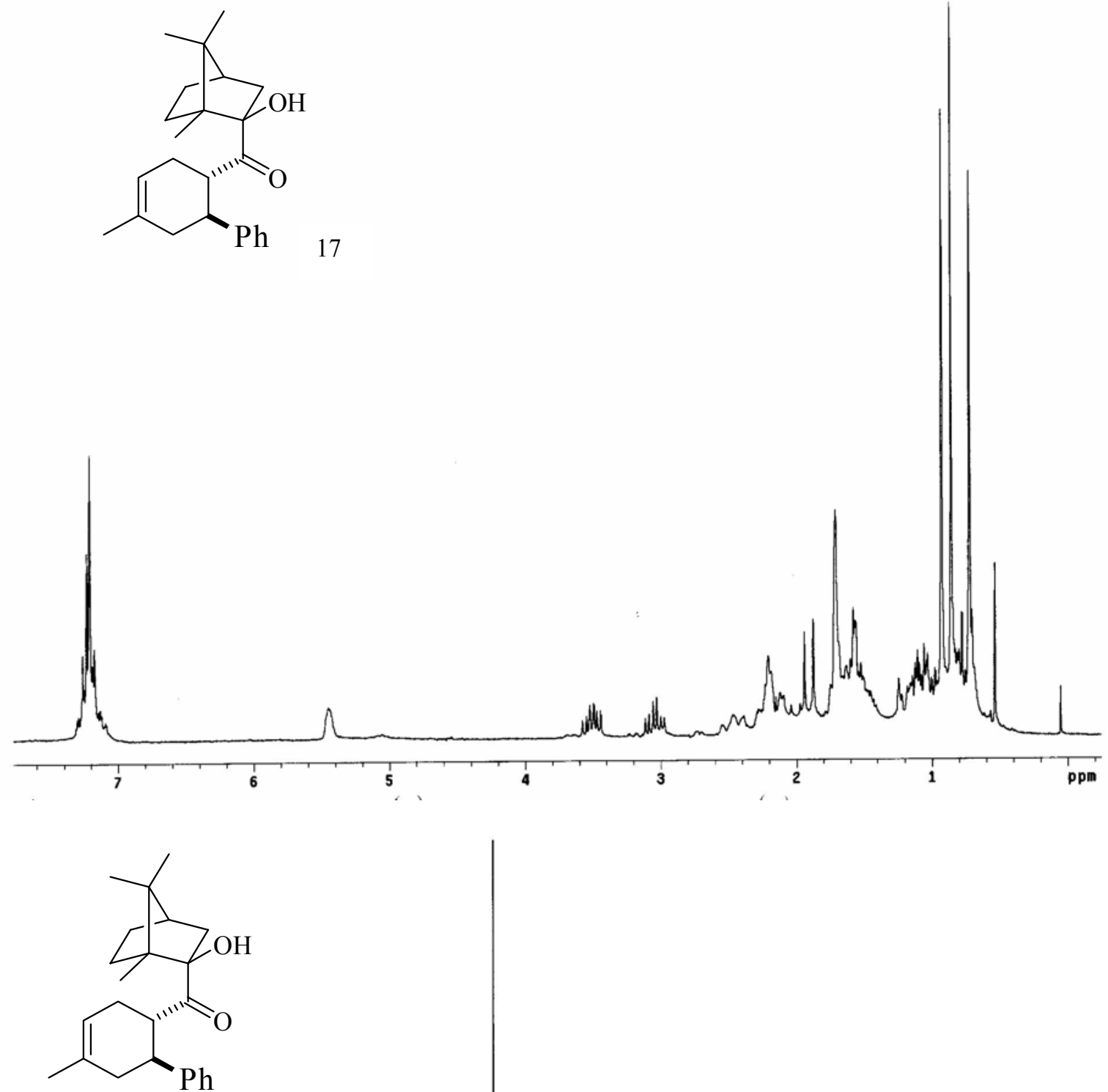

17

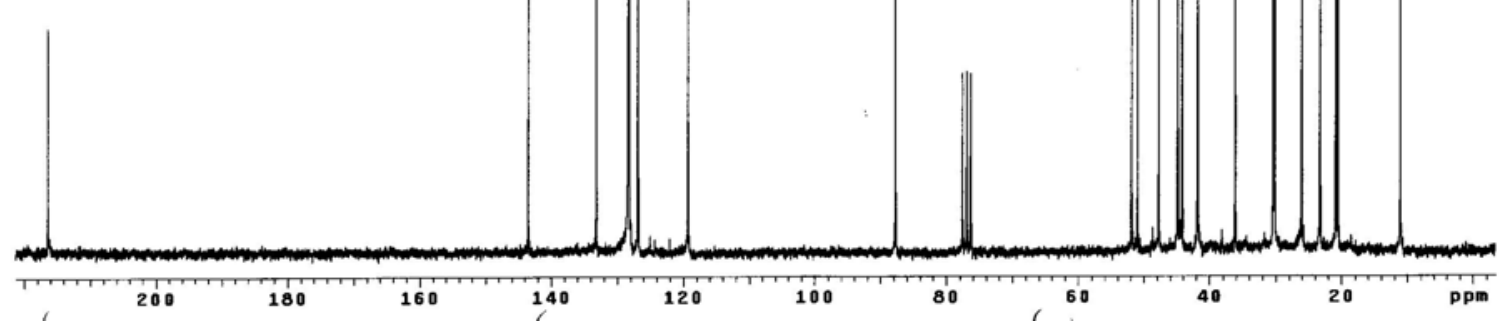



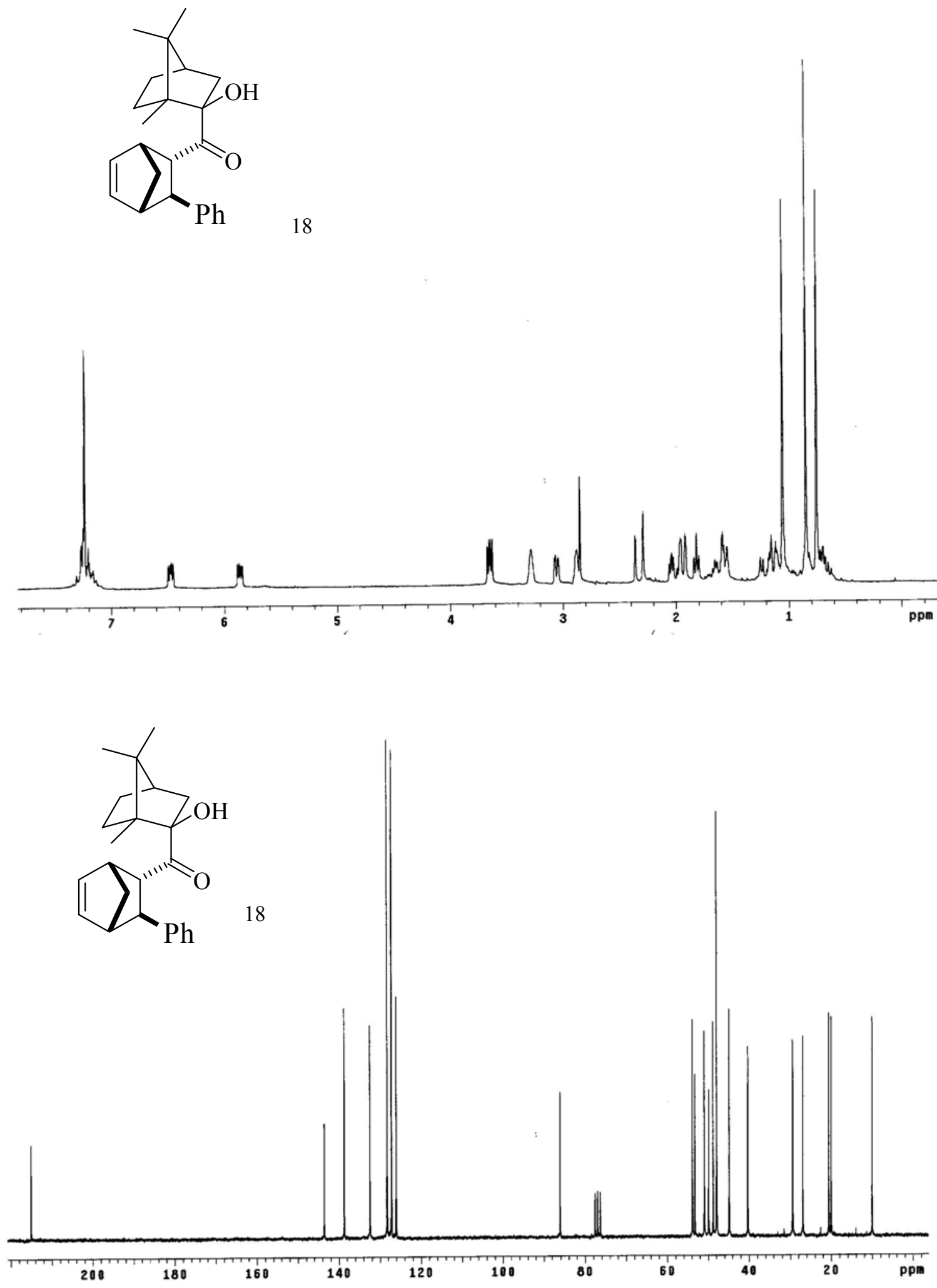

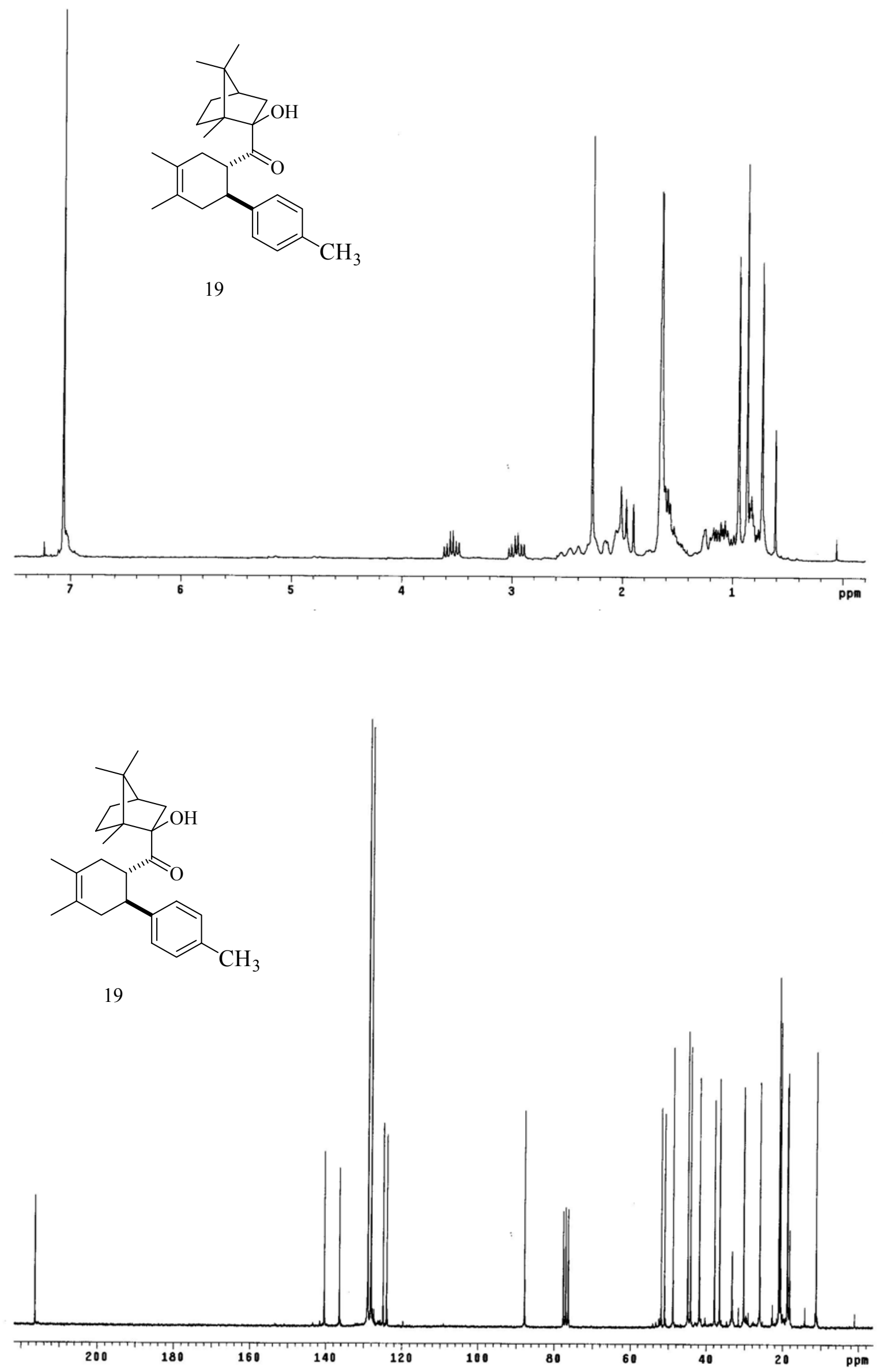

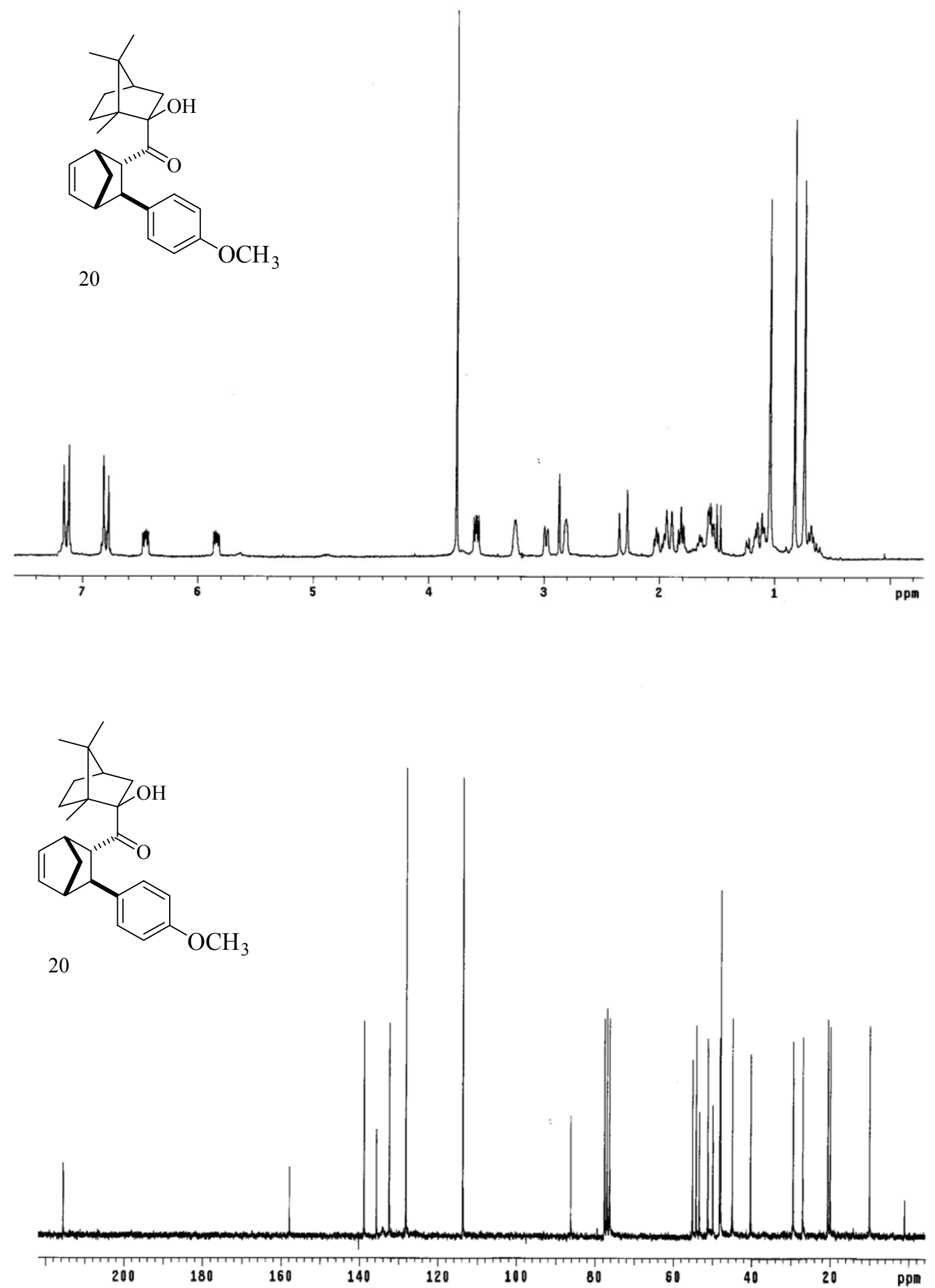


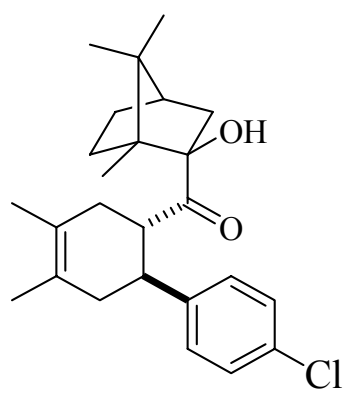

21
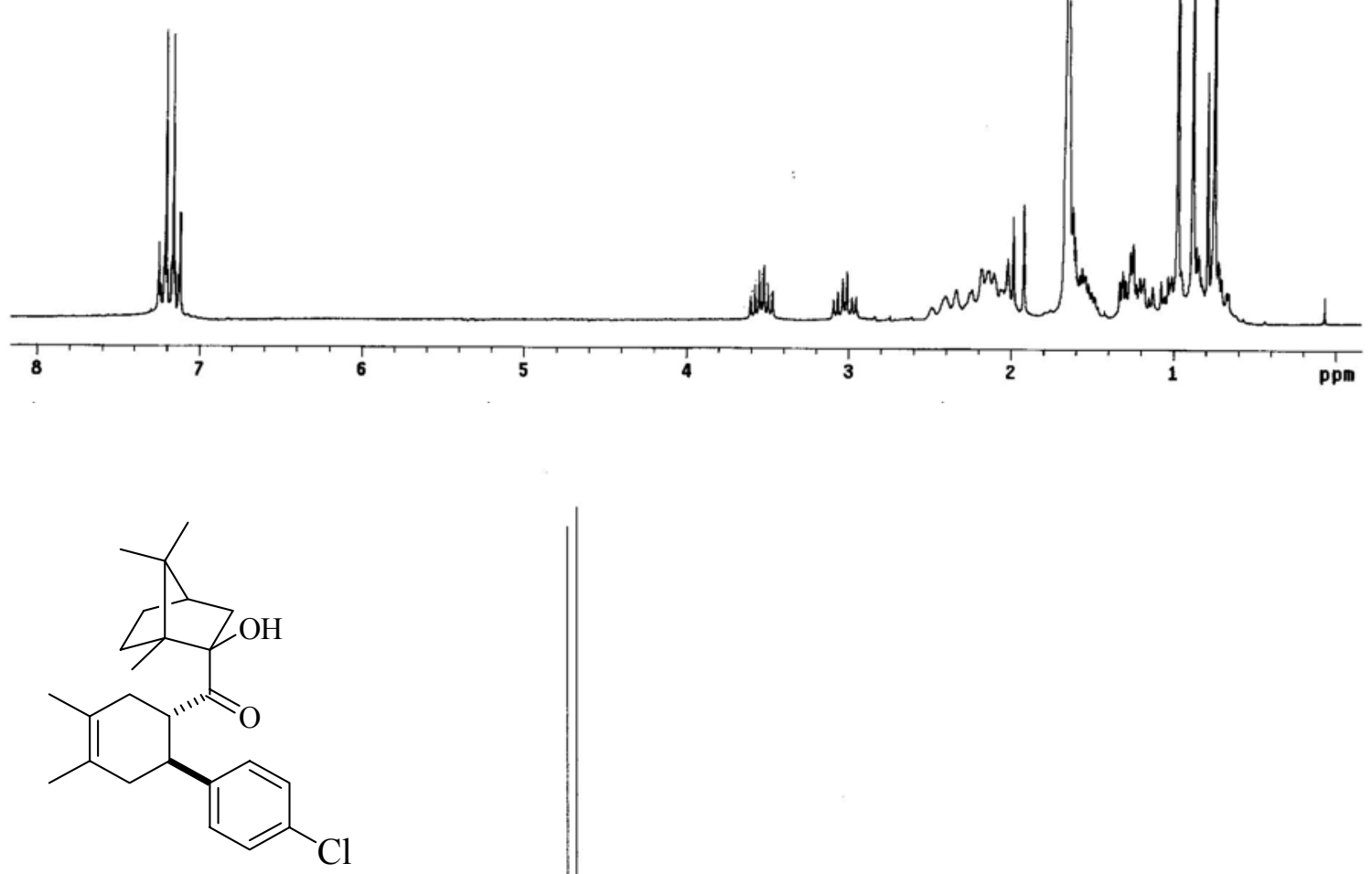

21

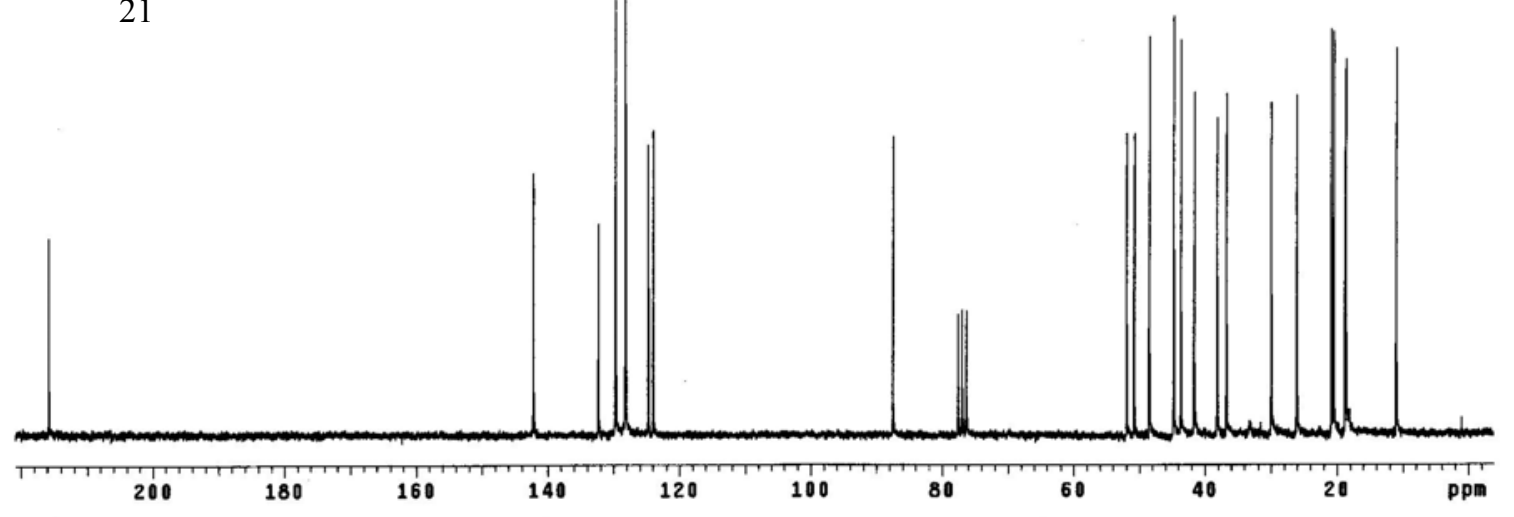


<smiles>CC1=CC[C@H](C(=O)O)CC1</smiles>

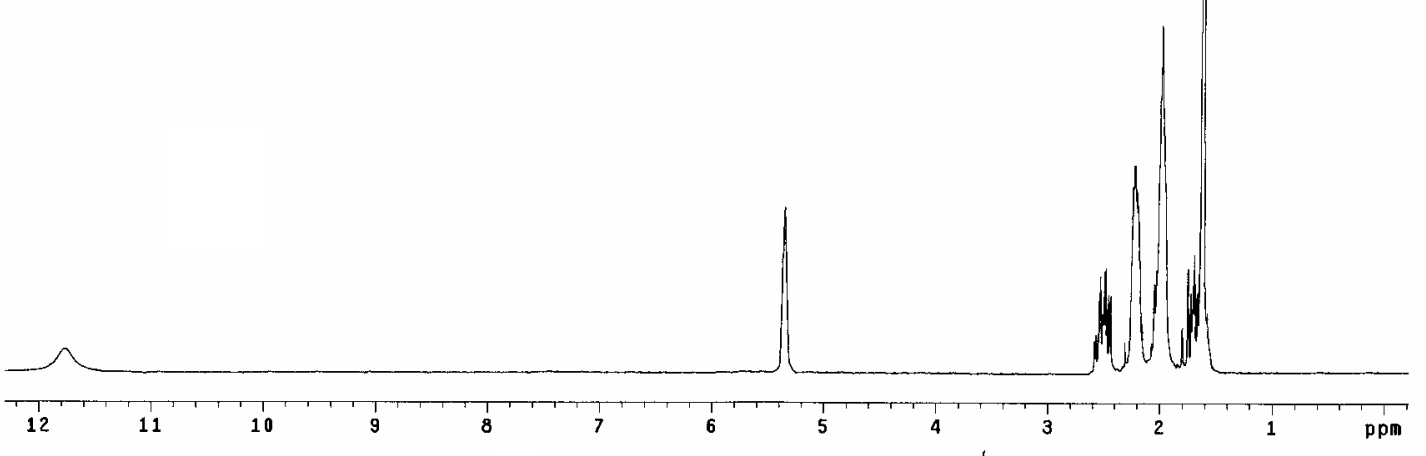<smiles>CC1=CC[C@H](C(=O)O)CC1</smiles>

22

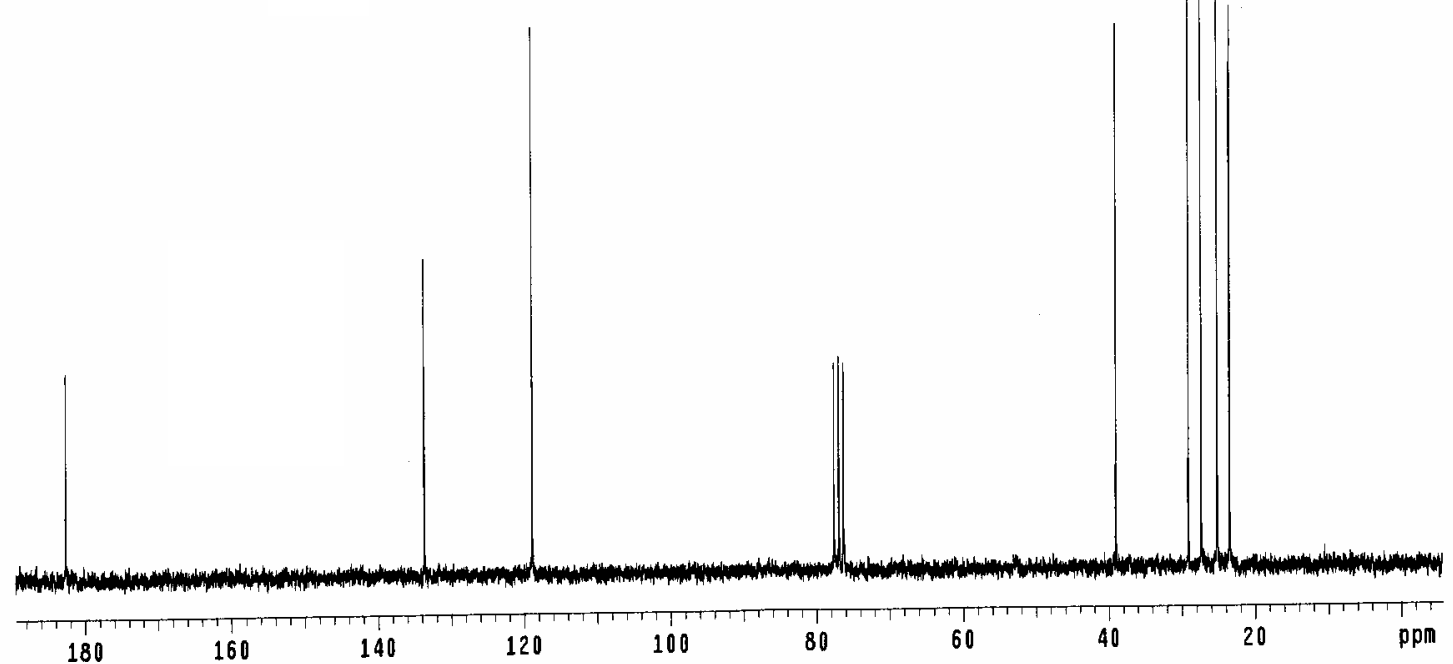


$D^{\prime \prime C O} \mathrm{CO}_{2} \mathrm{H}$

23

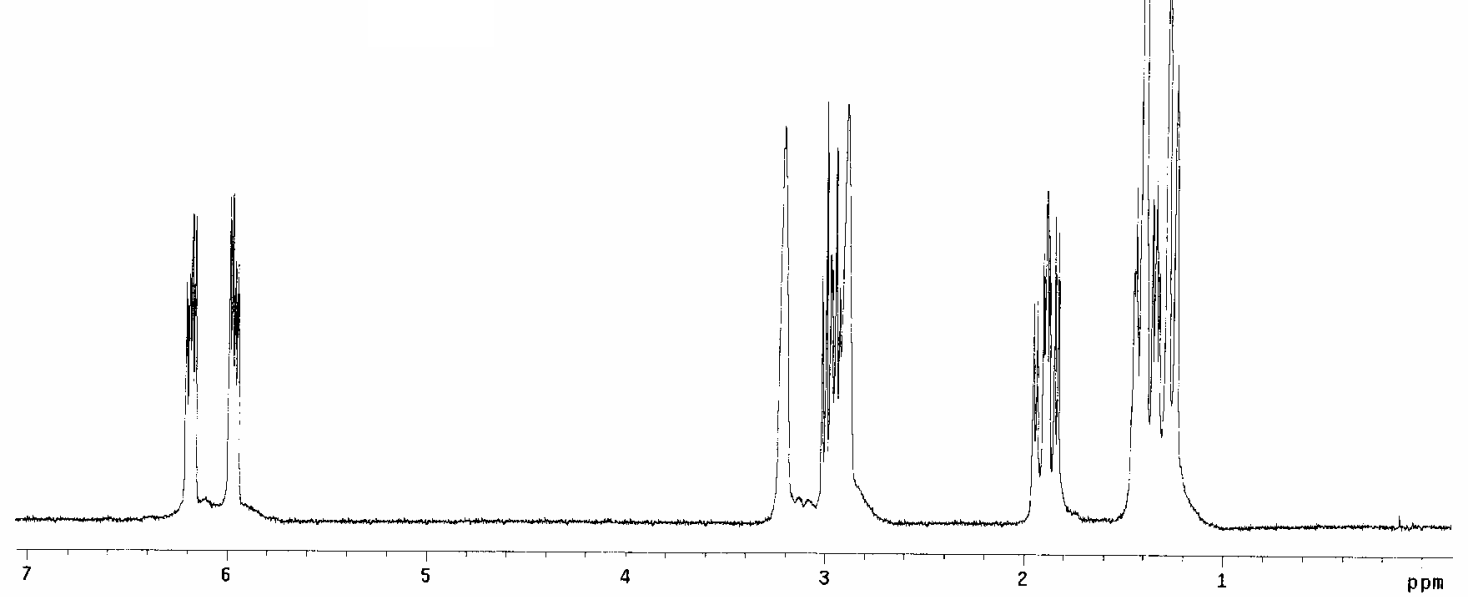

$\int_{23}^{\prime \prime \prime C_{2} \mathrm{H}}$

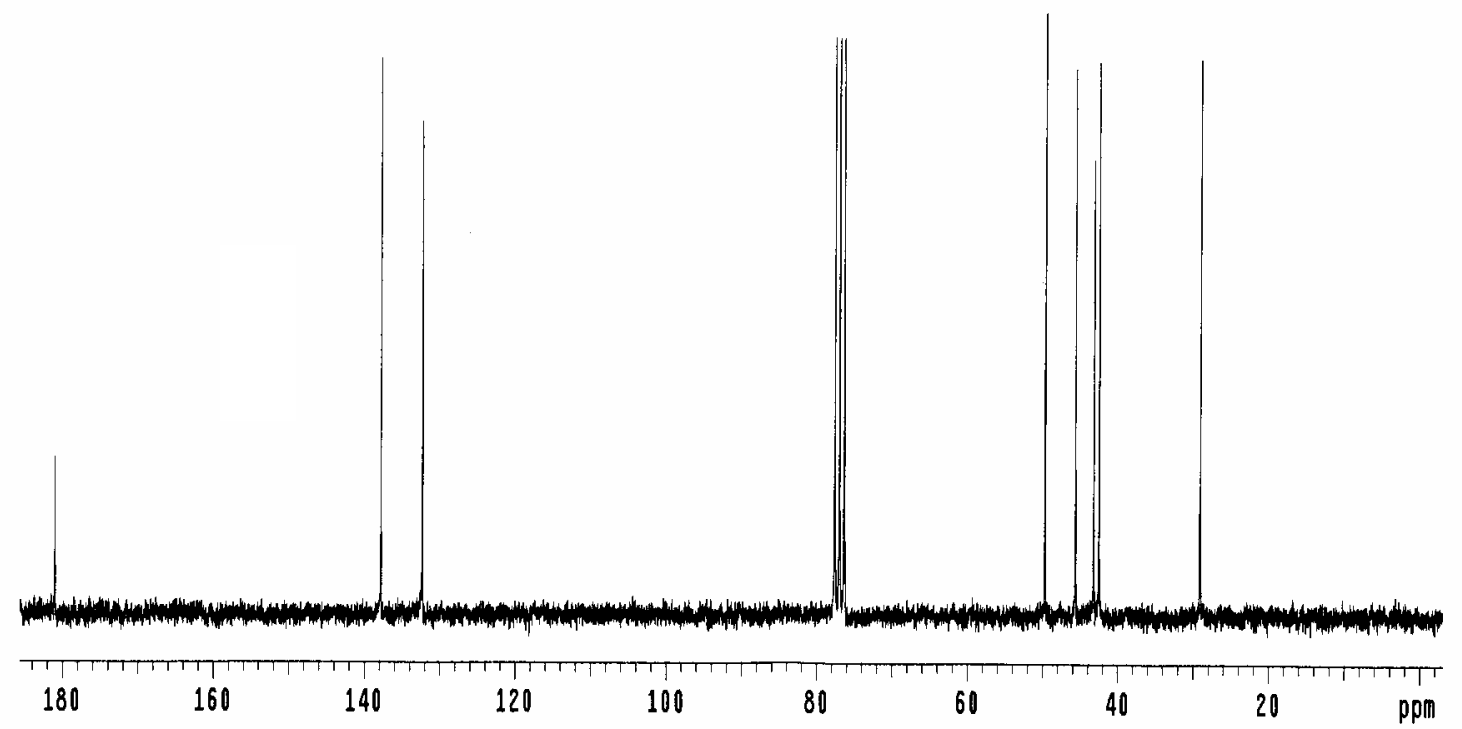



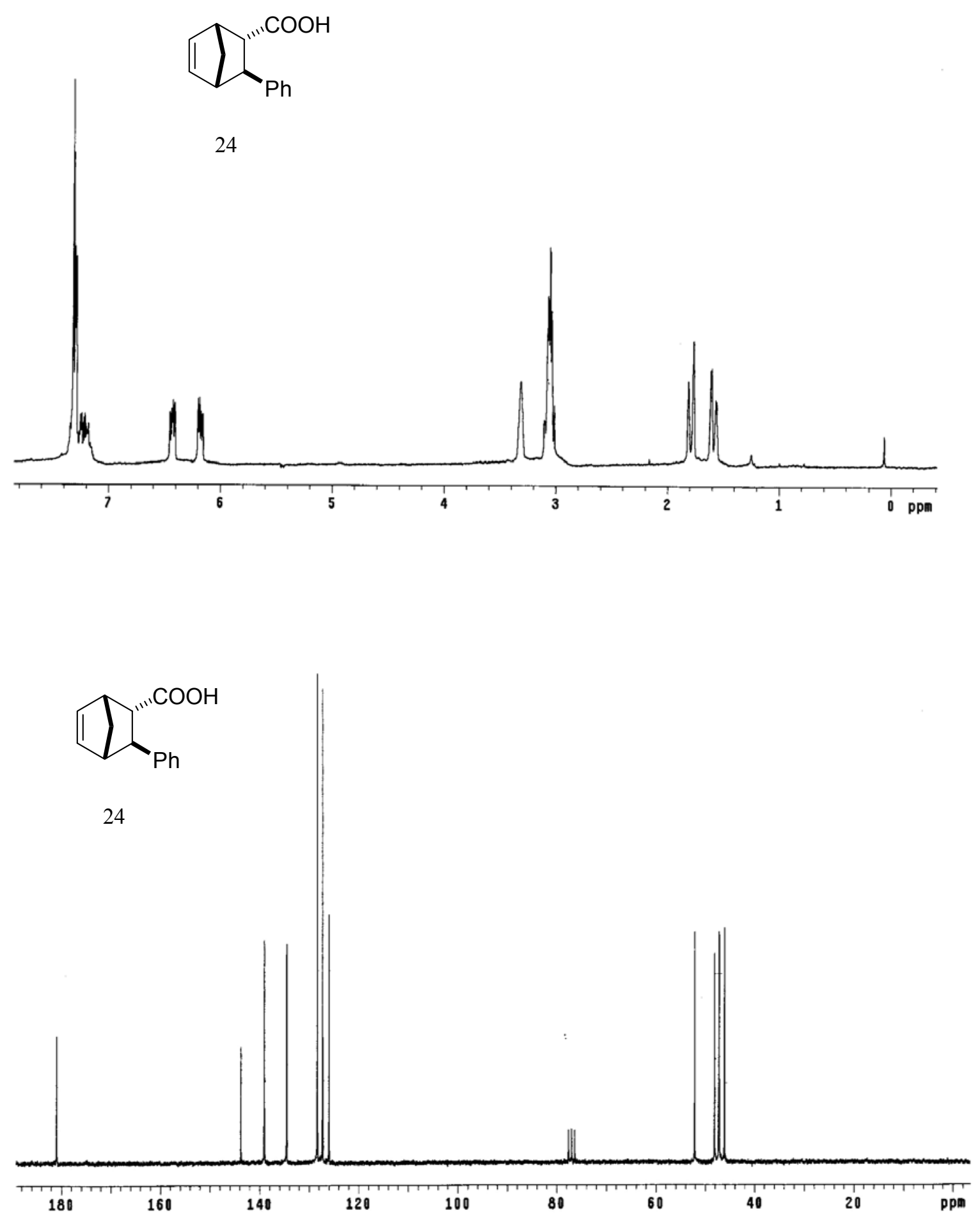
<smiles>CC(=O)[C@H]1CC=C(C)CC1</smiles>

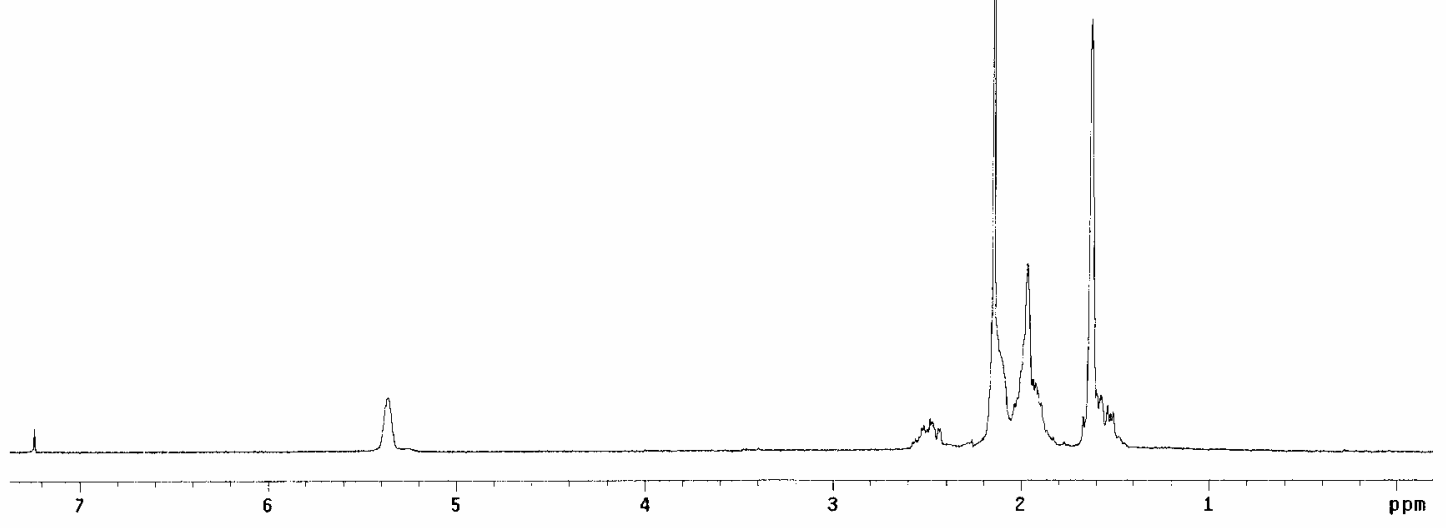<smiles>CC(=O)[C@H]1CC=C(C)CC1</smiles>

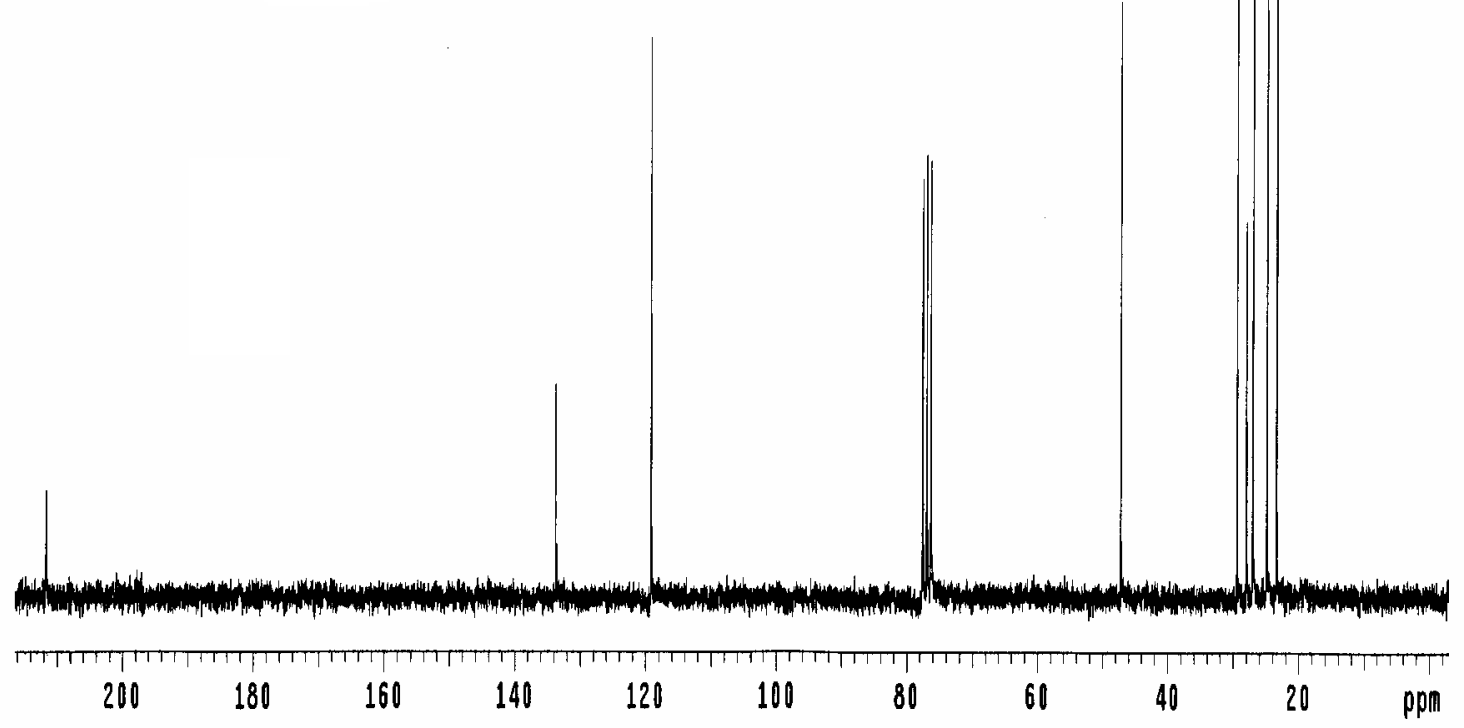


<smiles>CCCCC(=O)[C@H]1CC=C(C)CC1</smiles>

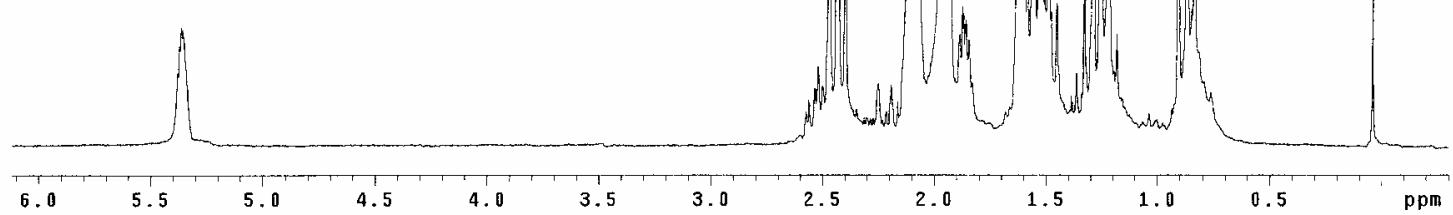<smiles>CCCCC(=O)[C@H]1CC=C(C)CC1</smiles>

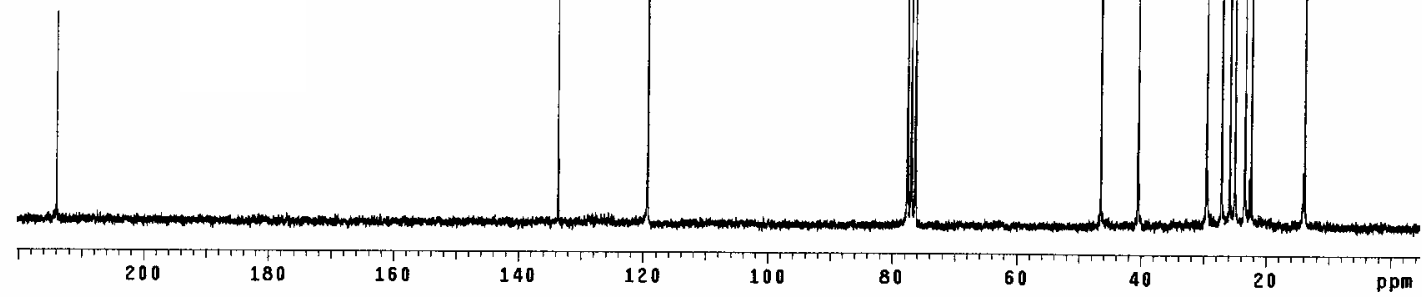




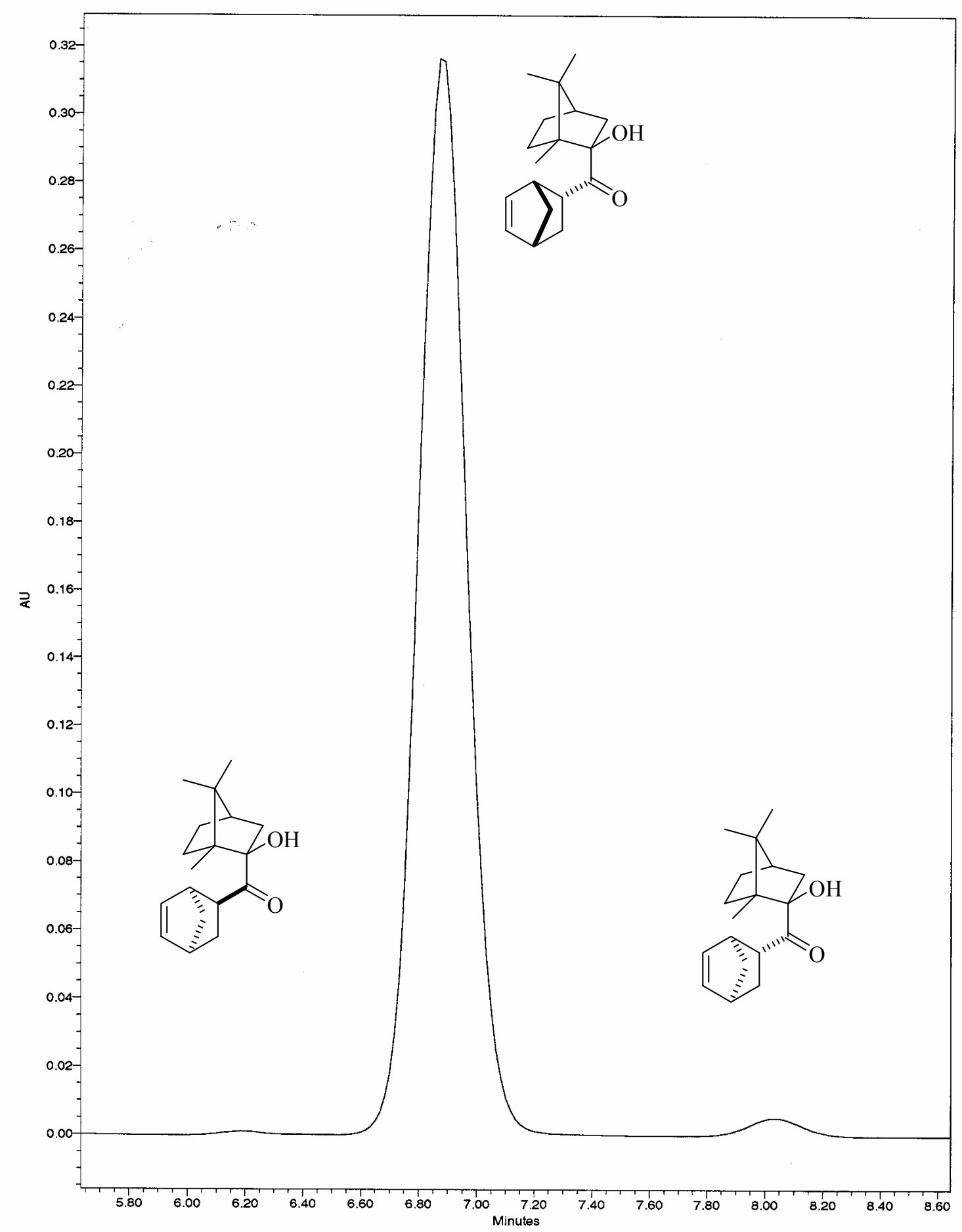

\begin{tabular}{|r|r|r|r|r|r|r|r|l|l|l|}
\hline 2. & 6.184 & 7909 & 0.21 & 895 & $\mathrm{bb}$ & & & Unknown & \\
\hline 2. & 6.874 & 3722540 & 98.14 & 317797 & $\mathrm{bb}$ & & & Unknown & \\
\hline
\end{tabular}




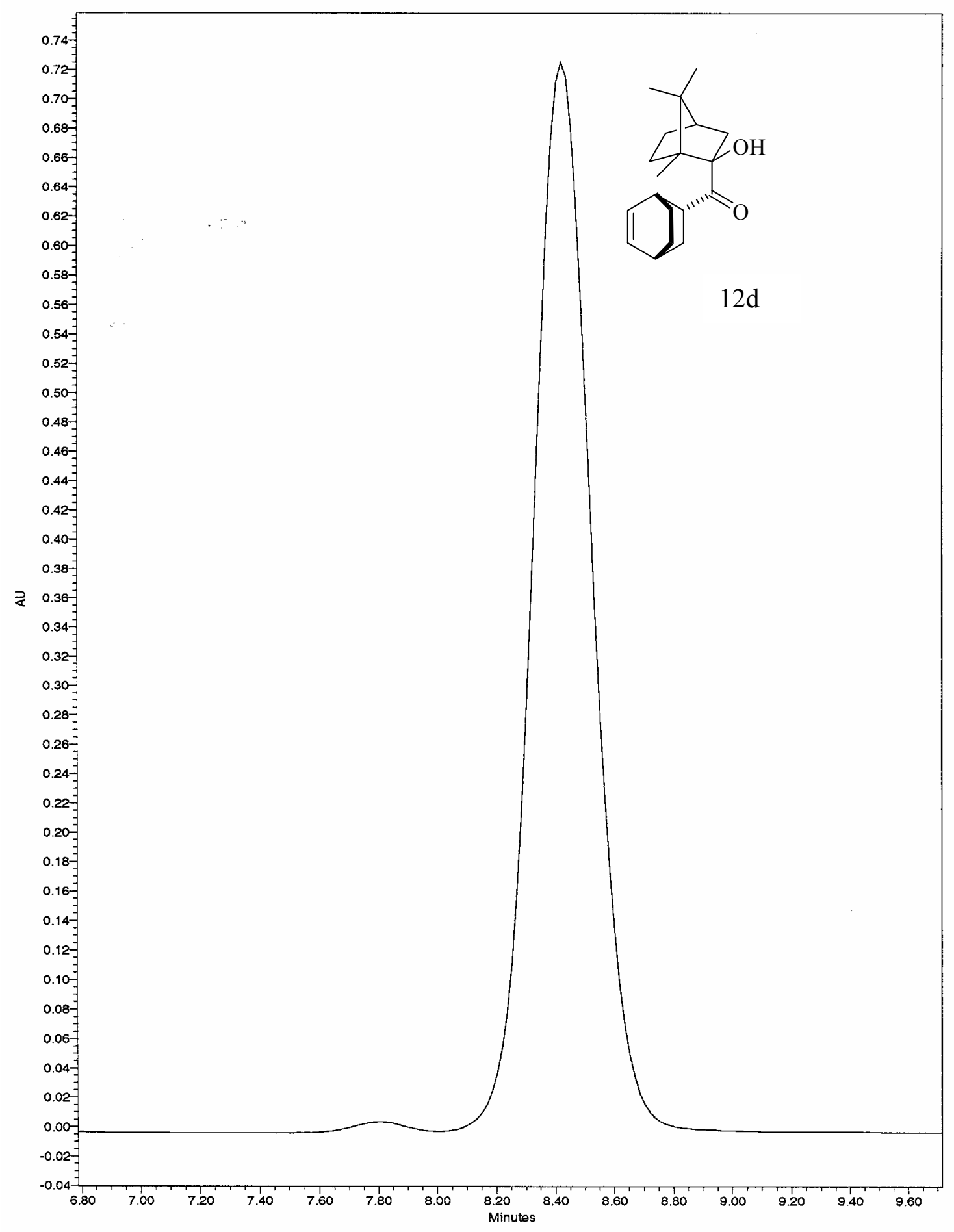

\begin{tabular}{|c|c|c|c|c|c|c|c|c|c|}
\hline S & 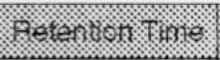 & sies & \% & 1) & 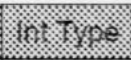 & 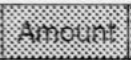 & xins & 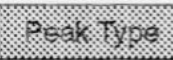 & Sodes \\
\hline : & 7.803 & 62259 & 0.61 & 6023 & $\mathrm{bb}$ & & & Unknown & \\
\hline 2. & 8.422 & 10169522 & 99.39 & 727853 & $\mathrm{bb}$ & & & Unknown & \\
\hline
\end{tabular}



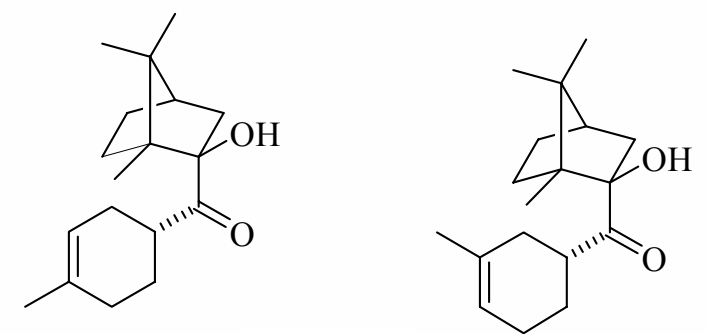

70:30
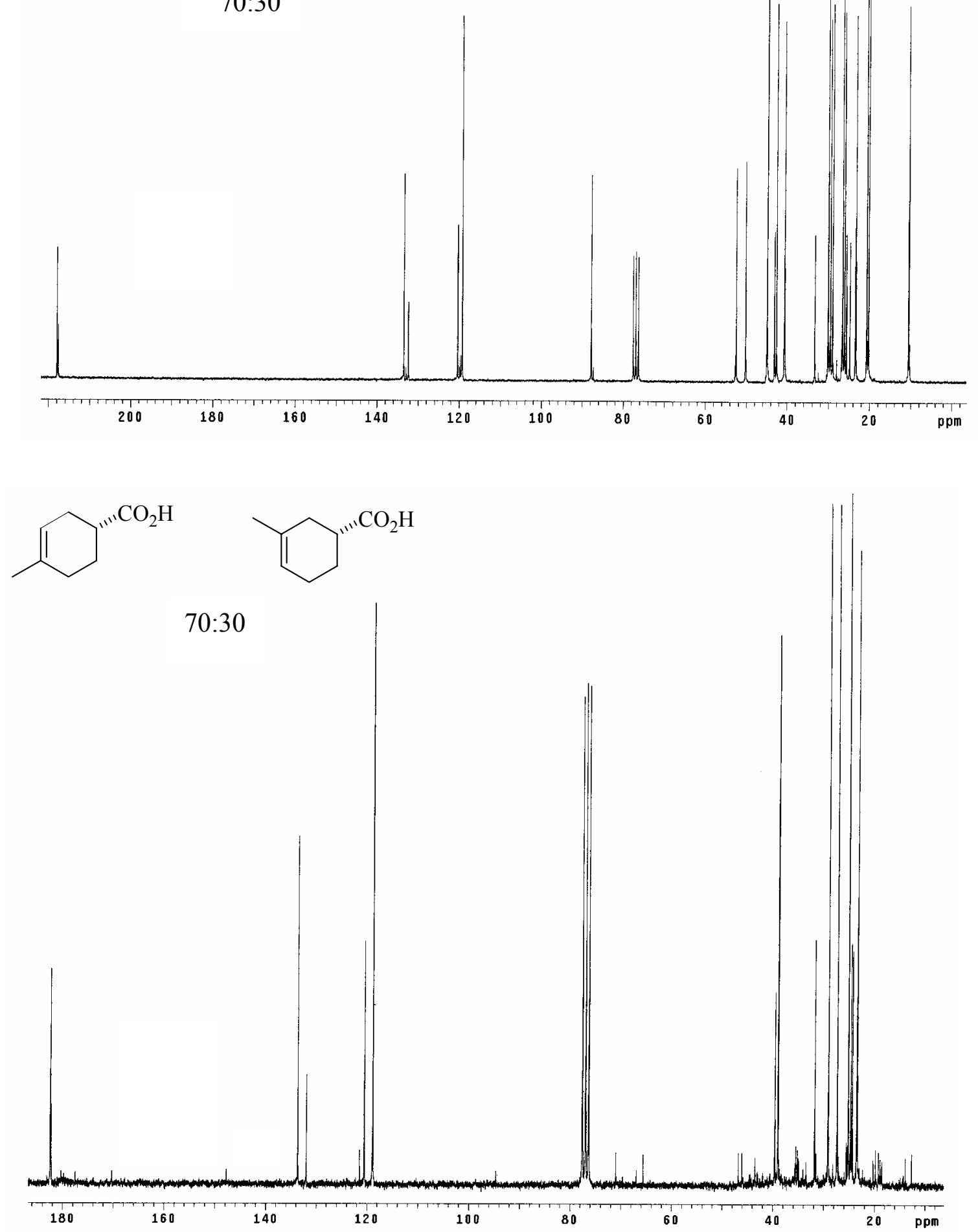
<smiles>CC1=CC[C@H](C(=O)C2(O)CC3CCC2C3(C)C)CC1</smiles><smiles>CC1=CCC[C@H](C(=O)C2(O)CC3CCC2C3(C)O)C1</smiles>

94:6

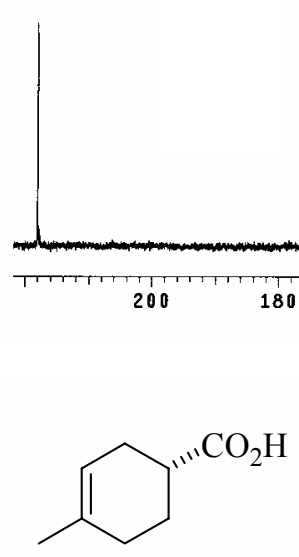<smiles>CC1=CCC[C@H](C(=O)O)C1</smiles>

94:6

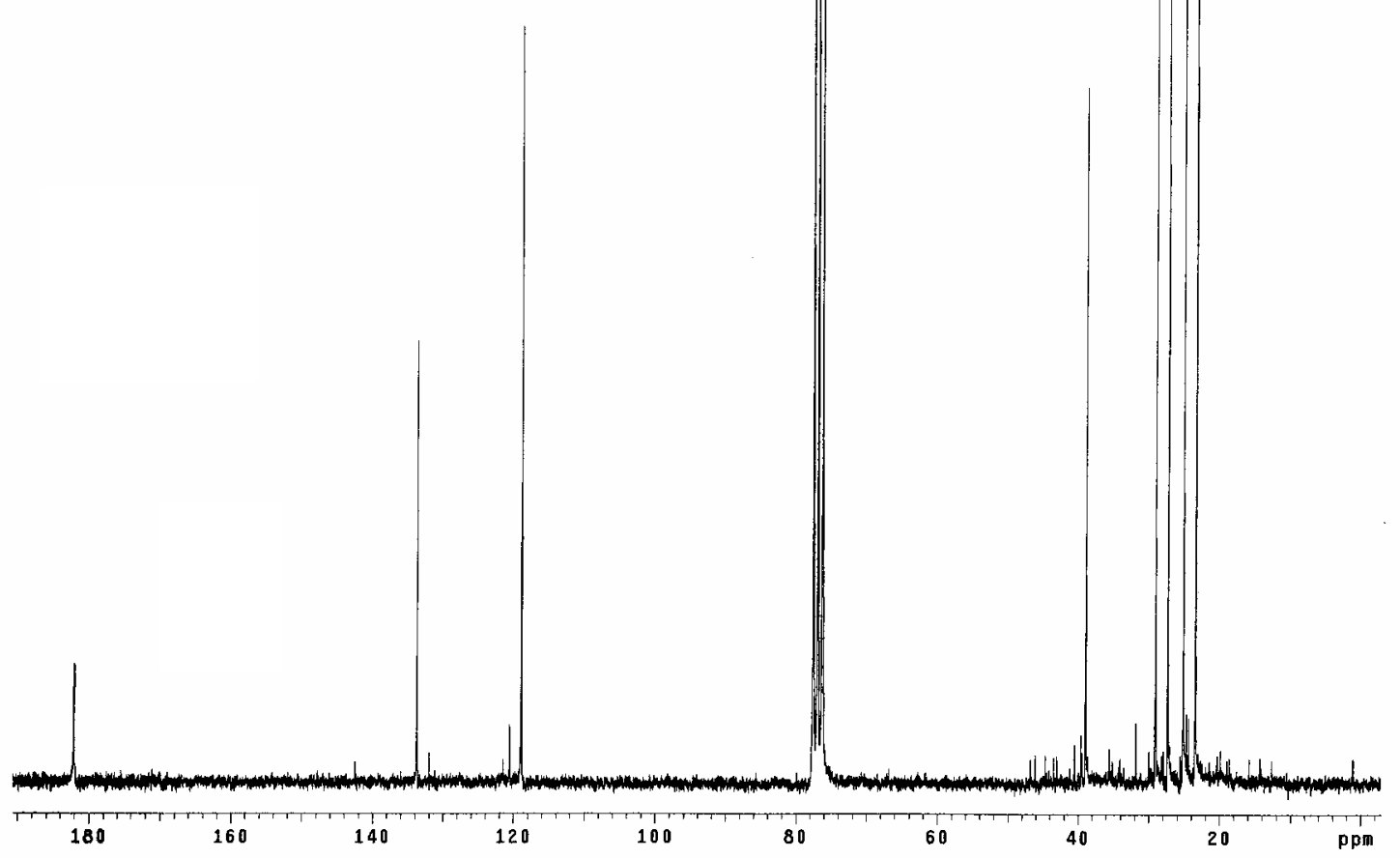



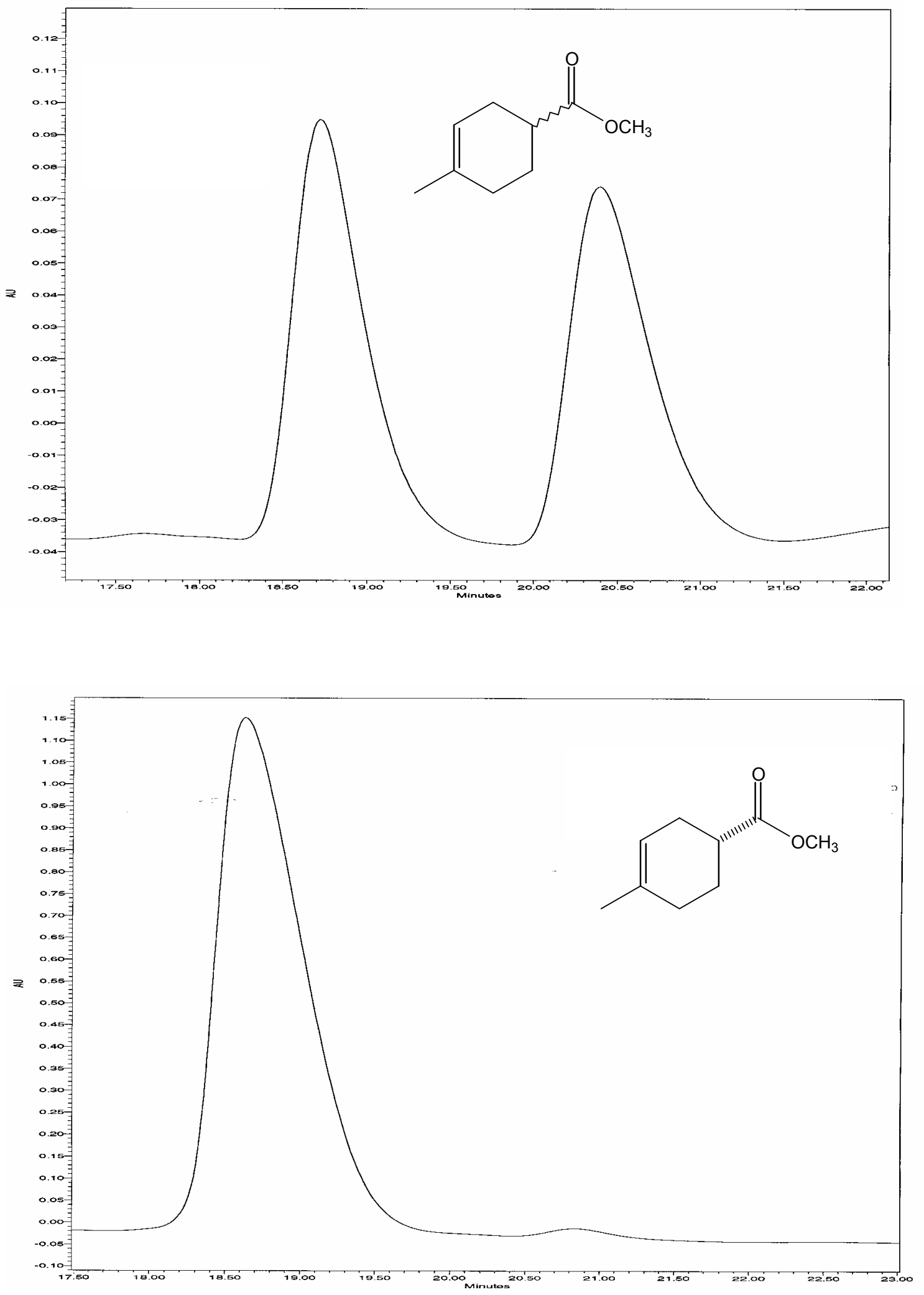

\begin{tabular}{|r|r|r|r|r|l|l|l|l|l|l|}
\hline & 18.626 & 39971631 & 99.00 & 1006922 & bb & & & Unknown & \\
\hline & 20.828 & 405176 & 1.00 & 15894 & bb & & & Unknown & \\
\hline
\end{tabular}



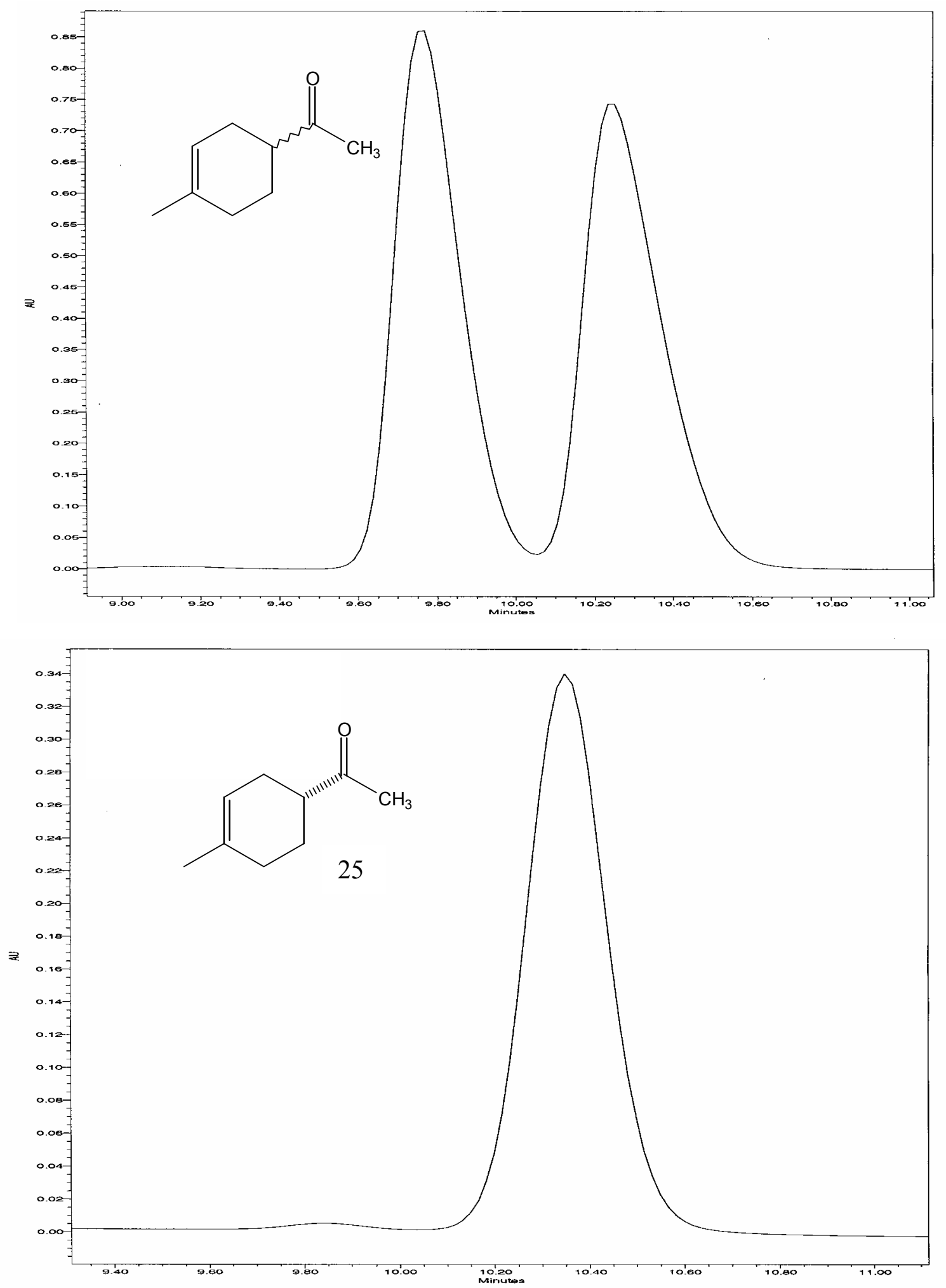

\begin{tabular}{|c|c|c|c|c|c|c|c|c|c|}
\hline Vivin: & 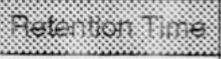 & $4.9=$ & 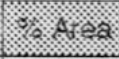 & is:ogtion & (I): $:$ : & (2) & (3):3:3it: & (2) o. & Co, 008 \\
\hline 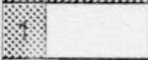 & 9.841 & 29414 & 0.96 & 2801 & $\mathrm{bb}$ & & & Unknown & \\
\hline 2 & 10.350 & 3031942 & 99.04 & 251997 & $b b$ & & & Unknown & \\
\hline
\end{tabular}



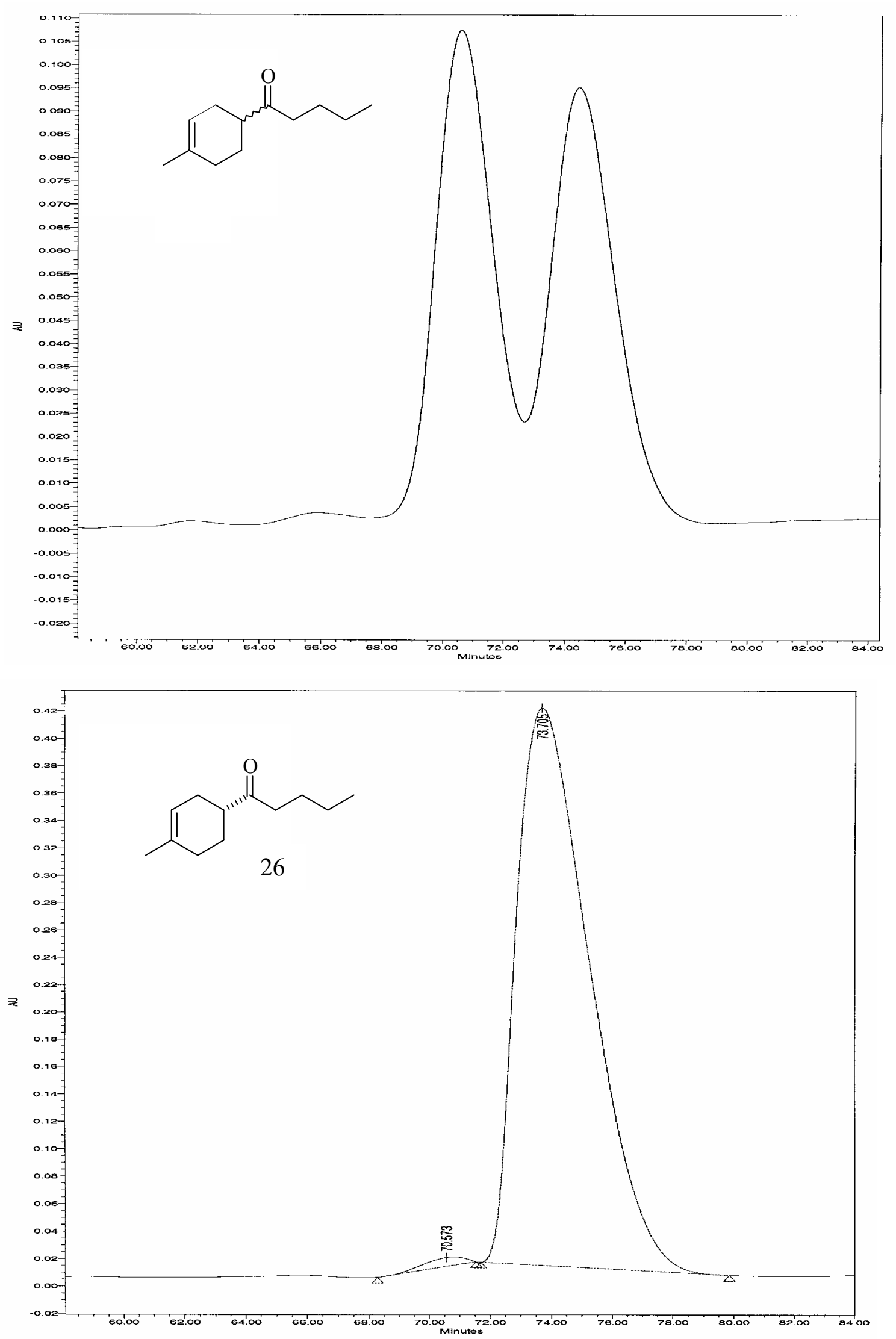

\begin{tabular}{|r|r|r|r|r|r|r|l|l|l|}
\hline & 70.573 & 603511 & 0.88 & 6427 & bb & & & Unknown & 108 \\
\hline
\end{tabular}


